\title{
Geometric analysis on Cantor sets and trees
}

\author{
Anders Björn \\ Department of Mathematics, Linköpings universitet, \\ SE-58183 Linköping, Sweden; anders.bjorn@liu.se \\ Jana Björn \\ Department of Mathematics, Linköpings universitet, \\ SE-58183 Linköping, Sweden; jana.bjorn@liu.se \\ James T. Gill \\ Department of Mathematics and Computer Science, Saint Louis University, \\ 220 N. Grand Blvd, St. Louis, MO 63103, U.S.A.; jgill5@slu.edu \\ Nageswari Shanmugalingam \\ Department of Mathematical Sciences, University of Cincinnati, \\ P.O. Box 210025, Cincinnati, OH 45221-0025, U.S.A.; shanmun@uc.edu
}

\begin{abstract}
Using uniformization, Cantor type sets can be regarded as boundaries of rooted trees. In this setting, we show that the trace of a first-order Sobolev space on the boundary of a regular rooted tree is exactly a Besov space with an explicit smoothness exponent. Further, we study quasisymmetries between the boundaries of two trees, and show that they have rough quasiisometric extensions to the trees. Conversely, we show that every rough quasiisometry between two trees extends as a quasisymmetry between their boundaries. In both directions we give sharp estimates for the involved constants. We use this to obtain quasisymmetric invariance of certain Besov spaces of functions on Cantor type sets.
\end{abstract}

Key words and phrases: Besov space, Cantor set, doubling measure, extension, metric space, Newtonian space, Poincaré inequality, quasisymmetry, rough quasiisometry, Sobolev space, totally disconnected, trace, tree, ultrametric, uniformly perfect, visual boundary.

Mathematics Subject Classification (2010): Primary: 30L10; Secondary:, 30L05, 31E05, 46E35, 51M10.

\section{Introduction}

Much of the recent development of analysis in metric measure spaces has tended to focus on two types of metric measure spaces: those that are highly connected (whose measures are doubling and support a Poincaré inequality, see for example [4], [5], [6], [7], [10], [11], [19], [22], [25], [28], [37], [44], and the references therein), and those that are fractals with a minimal connectedness property (the so-called postcritically finite fractals such as the Sierpiński gasket, see for example [13], [29], [42], [43], and the references therein). Totally disconnected sets such as Cantor sets tend to fall outside of both these categories. Papers such as Bellissard-Pearson [2] and Kigami [36] have studied analysis on Cantor type metric spaces, but only from the 
point of view of linear theory $(p=2)$. The goal of this paper is to study such totally disconnected sets from the point of view of nonlinear analysis on metric measure spaces, with emphasis on function spaces and on quasisymmetric mappings between such sets.

The setting considered here is that of uniformly perfect totally disconnected metric measure spaces, including various types of Cantor sets. As explained in Semmes [40], [41] such spaces are, up to biLipschitz equivalence, also obtained as ultrametric spaces which are boundaries of rooted regular trees equipped with a weighted metric (called uniformization metric in [8]). This point of view is similar to the uniformization process considered by Bonk-Heinonen-Koskela [8], and to the hyperbolic buildings, obtained by pasting together hyperbolic regions in a combinatorial way, studied by e.g. Bourdon-Pajot [10], [11]. However, while the Gromov boundaries in [8] as well as boundaries of hyperbolic buildings considered in [10] and [11] are highly connected, the boundary Cantor sets considered in this paper are totally disconnected. This in particular means that the Besov spaces considered below are nontrivial for all smoothness exponents $\theta>0$, in contrast to e.g. Théorème 0.3 in [11]. Note also that, because of the essentially one-dimensional structure of the trees, our setting does not fall under the scope of spaces with $Q$-bounded geometry considered with $Q>1$ in [8, Section 9].

Cantor sets embedded in Euclidean spaces support a fractional Sobolev space theory based on Besov spaces. Indeed, Besov functions on such sets are traces of the classical Sobolev functions on the ambient Euclidean spaces, see JonssonWallin [33], [34]. See also Danielli-Garofalo-Nhieu [14], [15] for such results on ambient Carnot-Carathéodory spaces. Similar extension and trace theorems on more general subsets of Euclidean spaces, obtained by means of Hajłasz-Sobolev type spaces on metric spaces, can be found in Hajłasz-Martio [23]. For further discussion of Sobolev functions on Euclidean domains and their extension and trace theorems we refer the reader to $\mathrm{Maz}^{\prime}$ ya [39].

Thus the potential theory on such Cantor sets is linked to the classical potential theory on the ambient Euclidean space. In the first part of this paper we obtain similar trace and extension theorems for Sobolev and Besov spaces on regular trees and their Cantor type boundaries. In particular, we show that the Besov space $B_{p, p}^{\theta}$ on the boundary is exactly the trace of the Newton-Sobolev space $N^{1, p}$ on the associated regular tree. Here the smoothness exponent of the Besov space is

$$
\theta=1-\frac{\beta / \varepsilon-Q}{p},
$$

where $Q$ is the Hausdorff dimension of the Cantor type boundary and $\beta / \varepsilon$ is a "dimension" determined by the uniformization metric and a weighted measure on the tree, see Propositions 6.1 and 6.4 and Theorem 6.5. In the setting considered here, we necessarily have $\beta / \varepsilon>Q$ as stipulated in (3.2), and so we have $\theta<1$. This is in contrast to Bourdon-Pajot [11], where, when $p<Q$, one needs $\theta=Q / p>1$.

The trace theorem we obtain in this paper corresponds exactly to the exponents in Jonsson-Wallin [33], [34]. For trees, our result extends and complements the general trace result for Besov spaces on metric spaces in Gogatishvili-KoskelaShanmugalingam [19, Theorem 6.5]. As an application of our trace result, for sufficiently large $p$ we obtain embeddings of Besov spaces on Cantor sets into spaces of Hölder continuous functions, see Proposition 6.6. Along the way we also show that trees with bounded degree, equipped with a weighted metric and measure (called a uniformization metric in [8]) are doubling and support a 1-Poincaré inequality, see Sections 3 and 4.

In Bourdon-Pajot [11, Théorème 0.1] certain Besov spaces with the smoothness exponent $\theta=Q / p$ were identified with cohomologies of conformal gauges. As a 
special case of the above trace theorem, we obtain a variant of this result in our setting of totally disconnected Cantor type boundaries, see the comment following Proposition 6.1.

In the Euclidean setting it is now well known that quasiconformal mappings preserve the classical Sobolev spaces $W^{1, n}\left(\mathbb{R}^{n}\right)$, see the discussion in HeinonenKilpeläinen-Martio [26]. On totally disconnected spaces, quasiconformal mappings are not so useful, because of the lack of nonconstant curves. Instead, one should consider quasisymmetries, i.e. mappings satisfying

$$
\frac{d(f(x), f(y))}{d(f(x), f(z))} \leq \eta\left(\frac{d(x, y)}{d(x, z)}\right)
$$

for all $x, y, z$ with $x \neq z$, where $\eta:[0, \infty) \rightarrow[0, \infty)$ is a homeomorphism. In the setting of quasisymmetric mappings between Ahlfors regular metric spaces, Koskela-Yang-Zhou [38, Theorem 5.1] recently obtained an invariance result for Besov spaces with the smoothness exponent $\theta=Q / p$, where $Q$ is the Ahlforsregularity dimension. Quasisymmetries also turn out to be the natural maps for studying boundaries of hyperbolic buildings and Gromov hyperbolic spaces, see for example [11], [17], [20], [30], [35], [46], and the references therein. Since trees are the quintessential models of Gromov hyperbolic spaces (see for example BridsonHaefliger [12]), these maps are natural for us as well.

Hence in the second part of this paper we consider quasisymmetries between the boundaries of (not necessarily regular) trees, and show that they can be extended to rough quasiisometries (also called quasiisometries in the literature) between the corresponding trees. A mapping $F$ between two trees is a rough quasiisometry if there are positive constants $L_{1}, L_{2}$ and $\Lambda$ such that for all points $x$ and $y$ in the domain tree,

$$
L_{1}|x-y|-\Lambda \leq|F(x)-F(y)| \leq L_{2}|x-y|+\Lambda,
$$

where $|\cdot-\cdot|$ denotes the unweighted geodesic distance on the tree, and the density condition that for each $z \in Y$ there is an $x \in X$ so that $|F(x)-z|<\Lambda$ holds. Conversely, we show that every rough quasiisometry between two trees induces a quasisymmetry between the respective boundaries. These results appear in Sections 8 and 9, and extend a theorem by Gromov [20] to our setting of trees with totally disconnected boundaries. The parameters $\eta, L_{1}$ and $L_{2}$ associated with the obtained mappings are optimal and match each other, see Theorems 8.2 and 9.9, and the comments following them.

The above extension result for quasisymmetries, together with our trace result for Besov spaces, is in turn used to show that certain Besov spaces on uniformly perfect ultrametric spaces are preserved by quasisymmetric mappings, see Theorem 8.3. For example, we show that every quasisymmetric $\left(\alpha_{1}, \alpha_{2}\right)$-power map as in (8.2) between two Cantor type spaces of Hausdorff dimensions $Q_{X}$ and $Q_{Y}$ induces the following embeddings between their Besov spaces,

$$
\begin{aligned}
B_{p, p}^{Q_{Y} / p+\tau / \alpha_{1}} \longleftrightarrow B_{p, p}^{Q_{X} / p+\tau} \longrightarrow B_{p, p}^{Q_{Y} / p+\tau / \alpha_{2}} \\
B_{p, p}^{Q_{Y} / p-\tau / \alpha_{2}} \longleftrightarrow B_{p, p}^{Q_{X} / p-\tau} \longleftrightarrow B_{p, p}^{Q_{Y} / p-\tau / \alpha_{1}}
\end{aligned}
$$

with $\tau \geq 0$, see Remark 8.4. This extends (in the setting of such spaces) the above mentioned Theorem 5.1 in Koskela-Yang-Zhou [38] beyond the case $\theta=Q / p$ considered there. Thus, potential theory on uniformly perfect ultrametric spaces is associated with the theory of quasisymmetric mappings between them. We also direct interested readers to Hambly-Kumagai [24] for a discussion linking rough quasiisometries (called rough isometries in [24]) to potential theory on graphs that arise as approximations of finitely ramified fractals. 
Acknowledgement. This research began when the first three authors visited the University of Cincinnati in 2010, and was continued during the visits of the fourth author to the University of Washington, Seattle, and to the Linköping University in 2011. The authors wish to thank these institutions for their kind hospitality. We also wish to thank David Minda for pointing out the reference Jeffers [32].

A. B. and J. B. were supported by the Swedish Research Council. A. B. was also a Fulbright scholar during his visit to the University of Cincinnati, supported by the Swedish Fulbright Commission, while J. B. was a Visiting Taft Fellow during her visit to the University of Cincinnati, supported by the Charles Phelps Taft Research Center at the University of Cincinnati. J. G. was supported by the University of Washington at the beginning of this work and is supported by NSF Mathematical Sciences Postdoctoral Research Fellowship DMS-1004721. N. S. was partly supported by the Taft Research Center, the Simons Foundation grant \#200474 and the NSF grant DMS-1200915.

\section{Notation and preliminaries}

A graph $G$ is a pair $(V, E)$, where $V$ is a set of vertices and $E \subset V \times V$ is a set of edges. We are interested in undirected graphs and consider $(x, y)$ and $(y, x)$ to be the same edge for $x, y \in V$. Two vertices $x, y \in V$ are neighbors if $(x, y) \in E$. The degree of a vertex equals the number of neighbors it has. We will be interested in infinite graphs, but all vertices will be required to have finite degree.

The graph structure gives rise to a natural well-known connectivity structure. A tree is a connected graph without cycles, or equivalently a graph such that for any pair of vertices $x, y \in V$ there is a unique path of distinct edges connecting $x$ to $y$. A graph (or tree) is made into a metric graph by considering each edge as a geodesic of length one.

We will only be interested in rooted trees. A rooted tree $X$ is a tree with a distinguished vertex called the root, which we will denote by 0. In Section 8 and later, when we deal with more than one tree, we denote the root of a tree $X$ by $0_{X}$.

For $x \in X$, let $|x|$ be the distance from the root 0 to $x$, that is, the number of edges in the geodesic from 0 to $x$. The geodesic connecting two vertices $x, y \in X$ is denoted by $[x, y]$, and its length (the number of edges it contains) is denoted $|x-y|$; note that if $x$ and $y$ are descendants of two different children of 0 , then $|x-y|=|x|+|y|$. We write $x<y$ if a vertex $y$ is a descendant of a vertex $x$ (that is, $|x|<|y|$ and $x$ lies in the geodesic connecting 0 to $y$ ), and more generally $x \leq y$ if the geodesic from 0 to $y$ passes through $x$; in this case $|x-y|=|y|-|x|$.

The neighbors of a vertex $x \in X$ are of two types: A parent $y$ of $x$ is the neighbor which is closer to the root, and all other neighbors are children of $x$. Each vertex has exactly one parent, except for the root itself which has none. We will mostly consider rooted trees such that each vertex other than the root has degree at least 3 , while the root 0 is expected to have degree at least 2 .

The most familiar rooted trees are binary trees in which each vertex has exactly two children. More generally a $K$-ary tree is a rooted tree such that each vertex has exactly $K$ children. Note that a $K$-ary tree is almost regular: all vertices but the root have degree $K+1$, whereas the root has degree $K$. In this paper we say that a tree is regular if it is $K$-ary for some integer $K \geq 1$.

As is customary, we say that $A \lesssim B$ and equivalently $B \gtrsim A$, if there is a constant $C>0$ (independent of the variables that $A$ and $B$ are functions of) such that $A \leq C B$. We also write $A \simeq B$ if $A \lesssim B \lesssim A$.

Let $\varepsilon>0$ be fixed from now on. We introduce a uniformizing metric (in the 
sense of Bonk-Heinonen-Koskela [8]) on $X$ by

$$
d_{X}(x, y)=\int_{[x, y]} e^{-\varepsilon|z|} d|z| .
$$

Here $d|z|$ stands for the measure which gives each edge Lebesgue measure 1, as we consider each edge to be an isometric copy of the open unit interval and the vertices are the points which close this interval. In this metric, diam $X=2 / \varepsilon$ if the root has at least two children and every vertex has at least one child. Though this metric defines a weighted metric on both the vertices and the edges (seen as copies of an open interval on the real line), we will typically only discuss the distance between vertices.

Throughout the paper we assume that $1 \leq p<\infty$.

\section{Doubling condition on trees}

In this section we assume that $X$ is a rooted tree such that each vertex has at least one and at most $K$ children. The proofs of the results in this section, however, are substantially simpler if we assume $K$-regularity of the tree. In Remark 3.10 we show how to remove the regularity assumption so that the results hold under the above generality.

The aim of this section is to show that the weighted measure

$$
d \mu(x)=e^{-\beta|x|} d|x|
$$

is doubling on $X$ (when equipped with the uniformizing metric $d_{X}$ ), where

$$
\beta>\log K
$$

is fixed from now on. (If $\beta \leq \log K$, then $\mu(X)=\infty$ for the regular $K$-ary tree by (3.6) below, and as $X$ is bounded, $\mu$ would not be doubling. This case is therefore not of interest to us. For nonregular trees we might have $\mu(X)<\infty$ even if $\beta \leq \log K$, but we do not consider this case here.)

We shall estimate the measure of balls in $X$ and show that it is doubling. Let $B(x, r)=\left\{y \in X: d_{X}(x, y)<r\right\}$ denote an (open) ball in $X$ with respect to the metric $d_{X}$. Also let $F(x, r)=\left\{y \in X: y \geq x\right.$ and $\left.d_{X}(x, y)<r\right\}$ be the downward directed "half ball". Note that $X=B(0,1 / \varepsilon)=F(0,1 / \varepsilon)$ and that $F(x, \infty)=F\left(x, e^{-\varepsilon|x|} / \varepsilon\right)$. We need to consider several cases depending on whether the radius $r$ is small compared with $|x|$ or not.

The following comparisons and estimates for "half balls" will be useful. We first state a simple algebraic lemma which will simplify our calculations.

Lemma 3.1. Let $\sigma>0$ and $t \in[0,1]$. Then

$$
\min \{1, \sigma\} t \leq 1-(1-t)^{\sigma} \leq \max \{1, \sigma\} t .
$$

Proof. Let $f(\tau)=\tau^{\sigma}$. If $\sigma \geq 1$, then $f$ is convex and for $0 \leq \tau \leq 1$,

$$
1-\tau \leq f(1)-f(\tau) \leq f^{\prime}(1)(1-\tau) .
$$

Letting $\tau=1-t$ gives the conclusion. The case $\sigma \leq 1$ is treated similarly.

Lemma 3.2. For every $x \in X$ and $r>0$ we have

$$
F(x, r) \subset B(x, r) \subset F(z, 2 r),
$$

where $z \leq x$ and

$$
|z|=\max \left\{|x|-\frac{1}{\varepsilon} \log \left(1+\varepsilon r e^{\varepsilon|x|}\right), 0\right\} .
$$


Proof. The first inclusion is clear and true for all $r$. As for the second inclusion, note first that if $r \leq\left(1-e^{-\varepsilon|x|}\right) / \varepsilon$ then

$$
|z|=|x|-\frac{1}{\varepsilon} \log \left(1+\varepsilon r e^{\varepsilon|x|}\right)
$$

and

$$
d_{X}(x, z)=\int_{|z|}^{|x|} e^{-\varepsilon t} d t=\frac{1}{\varepsilon} e^{-\varepsilon|x|}\left(e^{-\varepsilon(|z|-|x|)}-1\right)=r .
$$

At the same time, if $r \geq\left(1-e^{-\varepsilon|x|}\right) / \varepsilon$, then

$$
d_{X}(x, z)=d_{X}(x, 0)=\int_{0}^{|x|} e^{-\varepsilon t} d t=\frac{1}{\varepsilon}\left(1-e^{-\varepsilon|x|}\right) \leq r .
$$

Hence, for all $r>0$ and all $y \in B(x, r)$, we have

$$
d_{X}(y, z) \leq d_{X}(x, y)+d_{X}(x, z)<2 r .
$$

Clearly, $y \geq z$ for such $y$, which completes the proof.

Lemma 3.3. For $z \in X$ and $0<r \leq e^{-\varepsilon|z|} / \varepsilon$,

$$
\mu(F(z, r)) \simeq e^{(\varepsilon-\beta)|z|} r .
$$

Note that the upper estimate in Lemma 3.3 holds even if some vertices in $X$ have no children, i.e. if we allow finite branches.

Proof. Let $\rho>0$ be such that

$$
\int_{|z|}^{|z|+\rho} e^{-\varepsilon t} d t=\frac{1}{\varepsilon} e^{-\varepsilon|z|}\left(1-e^{-\varepsilon \rho}\right)=r .
$$

Note that for each $|z| \leq t \leq|z|+\rho$, the number of points $y \in F(z, r)$ with $|y|=t$ is approximately $K^{t-|z|}$. Hence

$$
\begin{aligned}
\mu(F(z, r)) & \simeq \int_{|z|}^{|z|+\rho} K^{t-|z|} e^{-\beta t} d t=\frac{K^{-|z|}}{\beta-\log K} e^{(\log K-\beta)|z|}\left(1-e^{(\log K-\beta) \rho}\right) \\
& =\frac{e^{-\beta|z|}}{\beta-\log K}\left(1-\left(1-\varepsilon r e^{\varepsilon|z|}\right)^{(\beta-\log K) / \varepsilon}\right)
\end{aligned}
$$

Lemma 3.1 with $t=\varepsilon r e^{\varepsilon|z|}$ implies that

$$
\mu(F(z, r)) \simeq e^{-\beta|z|} \varepsilon r e^{\varepsilon|z|} \simeq e^{(\varepsilon-\beta)|z|} r .
$$

Corollary 3.4. If $0<r \leq e^{-\varepsilon|x|} / \varepsilon$, then $\mu(B(x, r)) \simeq e^{(\varepsilon-\beta)|x|} r$.

Proof. Let $z$ be as in Lemma 3.2. If $z=0$ then $B(x, r) \subset F(0, r+\rho)$, where

$$
\rho=d_{X}(0, x)=\int_{0}^{|x|} e^{-\varepsilon t} d t=\frac{1}{\varepsilon}\left(1-e^{-\varepsilon|x|}\right) \leq r
$$

and $r+\rho \leq 1 / \varepsilon=e^{-\varepsilon|z|} / \varepsilon$. For $z>0$ we have

$$
2 r \leq \frac{e^{-\varepsilon|x|}\left(1+\varepsilon r e^{\varepsilon|x|}\right)}{\varepsilon}=\frac{e^{-\varepsilon|z|}}{\varepsilon} .
$$

Since in both cases $1 \leq e^{|x|-|z|} \leq\left(1+\varepsilon r e^{\varepsilon|x|}\right)^{1 / \varepsilon} \simeq 1$, the result now follows by applying Lemma 3.3 to $F(x, r)$ and $F(z, 2 r)$ (or $F(0, r+\rho)$ for $z=0)$. 
Lemma 3.5. Let $x \in X$ and

$$
\frac{e^{-\varepsilon|x|}}{\varepsilon} \leq r \leq \frac{1}{\varepsilon}\left(1-e^{-\varepsilon|x|}\right) .
$$

Then $\mu(B(x, r)) \simeq r^{\beta / \varepsilon}$.

Proof. By Lemma 3.2 and the second inequality in (3.7), we have $B(x, r) \subset F(z, \infty)=$ $F\left(z, e^{-\varepsilon|z|} / \varepsilon\right)$, where $0 \leq z \leq x$ is given by (3.4). Lemma 3.3 then yields

$$
\mu(F(z, \infty)) \lesssim e^{(\varepsilon-\beta)|z|} e^{-\varepsilon|z|} \simeq e^{-\beta|z|} .
$$

Now, the first inequality in (3.7) implies that $1+\varepsilon r e^{\varepsilon|x|} \leq 2 \varepsilon r e^{\varepsilon|x|}$. It then follows from (3.4) that

$$
e^{-\beta|z|}=e^{-\beta|x|}\left(1+\varepsilon r e^{\varepsilon|x|}\right)^{\beta / \varepsilon} \leq(2 \varepsilon r)^{\beta / \varepsilon} .
$$

Inserting this into (3.8) finishes the proof of the upper bound.

As for the lower bound we have, using (3.4) again, that

$$
\mu(B(x, r)) \geq \int_{|z|}^{|x|} e^{-\beta t} d t=\frac{e^{-\beta|x|}}{\beta}\left(\left(1+\varepsilon r e^{\varepsilon|x|}\right)^{\beta / \varepsilon}-1\right) .
$$

The function $f(t)=\left((1+t)^{\beta / \varepsilon}-1\right) / t^{\beta / \varepsilon}$ is monotone and $\lim _{t \rightarrow \infty} f(t)=1$. As $\varepsilon r e^{\varepsilon|x|} \geq 1$, this yields that $f\left(\varepsilon r e^{\varepsilon|x|}\right) \geq \min \{1, f(1)\} \simeq 1$. Hence

$$
\mu(B(x, r)) \gtrsim e^{-\beta|x|}\left(\varepsilon r e^{\varepsilon|x|}\right)^{\beta / \varepsilon} \simeq r^{\beta / \varepsilon} .
$$

Lemma 3.6. Let $x \in X$ and $d_{X}(x, 0)=\left(1-e^{-\varepsilon|x|}\right) / \varepsilon \leq r \leq 2 \operatorname{diam} X$. Then $\mu(B(x, r)) \simeq r$. In particular, if $x=0$ then this estimate holds for all $r \geq 0$.

Proof. We have $0 \in \overline{B(x, r)}$ by assumption, and hence $B(x, r) \subset F(0,2 r)$. It then follows from Lemma 3.3 that

$$
\mu(B(x, r)) \leq \mu(F(0,2 r)) \lesssim r .
$$

As for the lower bound, consider first the case $r<1 / \varepsilon$. As $0 \in \overline{B(x, r)}$, letting $\rho=-\log (1-\varepsilon r) / \varepsilon$, implies

$$
\mu(B(x, r)) \geq \int_{0}^{\rho} e^{-\beta t} d t=\frac{1-e^{-\beta \rho}}{\beta}=\frac{1}{\beta}\left(1-(1-\varepsilon r)^{\beta / \varepsilon}\right) .
$$

Lemma 3.1 then yields $\mu(B(x, r)) \gtrsim r$. If $1 / \varepsilon \leq r \leq 2 \operatorname{diam} X \leq 4 / \varepsilon$, then

$$
\mu(B(x, r)) \geq \mu\left(B\left(x, \frac{1}{5} r\right)\right) \simeq r .
$$

The following proposition follows from Corollary 3.4 together with Lemmas 3.5 and 3.6. Note that if $\beta=\varepsilon$, then $X$ becomes Ahlfors 1-regular, that is, $\mu(B(x, r)) \simeq$ $r$ for all $x \in X$ and $0<r \leq 2 \operatorname{diam} X$.

Proposition 3.7. Let $x \in X, 0<r \leq 2 \operatorname{diam} X$ and $R_{0}=e^{-\varepsilon|x|} / \varepsilon$. If $|x| \leq$ $(\log 2) / \varepsilon$ then $\mu(B(x, r)) \simeq r$. If $|x| \geq(\log 2) / \varepsilon$ then

$$
\mu(B(x, r)) \simeq \begin{cases}e^{(\varepsilon-\beta)|x|} r & \text { for } r \leq R_{0} \\ r^{\beta / \varepsilon} & \text { for } r \geq R_{0}\end{cases}
$$

Proof. If $|x| \leq(\log 2) / \varepsilon$ then $e^{(\varepsilon-\beta)|x|} \simeq 1$ and the result follows directly from Corollary 3.4 and Lemma 3.6. (Note that $e^{-\varepsilon|x|} \geq \frac{1}{2}$ in this case.)

If $|x| \geq(\log 2) / \varepsilon$ and $r<\left(1-e^{-\varepsilon|x|}\right) / \varepsilon$ then the estimate follows directly from Corollary 3.4 and Lemma 3.5. For $r \geq\left(1-e^{-\varepsilon|x|}\right) / \varepsilon \geq 1 / 2 \varepsilon$ we have by Lemma 3.6 that $\mu(B(x, r)) \simeq r \simeq 1 \simeq r^{\beta / \varepsilon}$. 
Corollary 3.8. The following dimension condition holds for all balls $B(x, r)$ and $B\left(x^{\prime}, r^{\prime}\right)$ with $x^{\prime} \in B(x, r)$ and $0<r^{\prime} \leq r$,

$$
\frac{\mu\left(B\left(x^{\prime}, r^{\prime}\right)\right)}{\mu(B(x, r))} \gtrsim\left(\frac{r^{\prime}}{r}\right)^{s}
$$

where $s=\max \{1, \beta / \varepsilon\}$ is the best possible.

Proof. Assume first that $x^{\prime}=x$. The general case $x^{\prime} \in B(x, r)$ will be taken care of later. Assume also that $r \leq 2 \operatorname{diam} X$.

If $|x| \leq(\log 2) / \varepsilon$, then $\mu(B(x, r)) \simeq r$ for all $0 \leq r \leq 2$ diam $X$, by Proposition 3.7, and (3.9) follows. Also, if $|x| \geq(\log 2) / \varepsilon$ and both $r^{\prime}$ and $r$ belong to the same interval in Proposition 3.7, then (3.9) follows directly from Proposition 3.7.

Let us therefore assume that $|x| \geq(\log 2) / \varepsilon$ and $r^{\prime} \leq R_{0} \leq r$. Then

$$
\frac{\mu\left(B\left(x, r^{\prime}\right)\right)}{\mu(B(x, r))} \simeq \frac{e^{(\varepsilon-\beta)|x|} r^{\prime}}{r^{\beta / \varepsilon}} \simeq \begin{cases}\left(\frac{R_{0}}{r^{\prime}}\right)^{\beta / \varepsilon-1}\left(\frac{r^{\prime}}{r}\right)^{\beta / \varepsilon}, & \text { if } \beta \geq \varepsilon, \\ \left(\frac{R_{0}}{r}\right)^{\beta / \varepsilon-1}\left(\frac{r^{\prime}}{r}\right), & \text { if } \beta \leq \varepsilon .\end{cases}
$$

Since $R_{0} / r^{\prime} \geq 1 \geq R_{0} / r$, (3.9) follows in this case as well. This also shows that (3.9) cannot hold for any $s<\max \{1, \beta / \varepsilon\}$.

Now, let $x^{\prime} \in B(x, r)$ and $0<r^{\prime} \leq r \leq \operatorname{diam} X$. Then $B(x, r) \subset B\left(x^{\prime}, 2 r\right)$ and hence by the above

$$
\frac{\mu\left(B\left(x^{\prime}, r^{\prime}\right)\right)}{\mu(B(x, r))} \geq \frac{\mu\left(B\left(x^{\prime}, r^{\prime}\right)\right)}{\mu\left(B\left(x^{\prime}, 2 r\right)\right)} \gtrsim\left(\frac{r^{\prime}}{2 r}\right)^{s} .
$$

Finally, if $r \geq \operatorname{diam} X$, then $B(x, r)=X=B\left(x^{\prime}, \operatorname{diam} X\right)$ and thus

$$
\frac{\mu\left(B\left(x^{\prime}, r^{\prime}\right)\right)}{\mu(B(x, r))}=\frac{\mu\left(B\left(x^{\prime}, r^{\prime}\right)\right)}{\mu\left(B\left(x^{\prime}, \operatorname{diam} X\right)\right)} \gtrsim\left(\frac{r^{\prime}}{\operatorname{diam} X}\right)^{s} \geq\left(\frac{r^{\prime}}{r}\right)^{s} .
$$

This immediately gives the following corollary.

Corollary 3.9. The measure $\mu$ is doubling, i.e. $\mu(B(x, 2 r)) \lesssim \mu(B(x, r))$.

Remark 3.10. Assume that $X$ is nonregular but such that each vertex has at least one and at most $K$ children for some $K \geq 1$, and let $\beta>\log K$. By adding edges to $X$ we obtain a regular $K$-ary tree $X_{K}$ containing $X$, equipped with the same metric and measure as $X$. For every $x \in X$ and $r>0$ we then have

$$
B(x, r) \subset B_{X_{K}}(x, r):=\left\{y \in X_{K}: d_{X}(x, y)<r\right\}
$$

At the same time, an infinite geodesic from 0 in $X$, passing through $x$, can be regarded as a regular 1-ary tree $X_{1}$ contained in $X$. Hence

$$
B(x, r) \supset B_{X_{1}}(x, r):=\left\{y \in X_{1}: d_{X}(x, y)<r\right\} .
$$

Since $X_{1}$ and $X_{K}$ are regular 1 -ary and $K$-ary trees, all results in this section apply to them and we have by Proposition 3.7,

$$
\mu\left(B_{X_{1}}(x, r)\right) \leq \mu(B(x, r)) \leq \mu\left(B_{X_{K}}(x, r)\right) \simeq \mu\left(B_{X_{1}}(x, r)\right) .
$$

This implies that the results in this section hold also for nonregular $X$ with bounds on the number of children. In particular, the measure $\mu$ given by (3.1) is doubling. 


\section{Poincaré inequality on trees}

In this section we assume that $X$ is a rooted tree such that each vertex has at most $K$ children (in particular, finite branches are allowed).

We will show that the measure and metric, given in Section 3, together support a 1-Poincaré inequality for functions and their upper gradients. Let $u \in L_{\mathrm{loc}}^{1}(X)$. We say that a Borel function $g: X \rightarrow[0, \infty]$ is an upper gradient of $u$ if

$$
|u(z)-u(y)| \leq \int_{\gamma} g d_{X} s
$$

whenever $z, y \in X$ and $\gamma$ is the geodesic from $z$ to $y$, where $d_{X} s$ denotes the arc length measure with respect to the metric $d_{X}$. In the current literature on metric measure spaces, the inequality (4.1) is required to hold for all rectifiable curves with end points $z$ and $y$. However, in the setting of a tree any such curve contains the geodesic connecting $z$ to $y$, and it is therefore equivalent to define it as above on the tree.

The notion of upper gradients is due to Heinonen and Koskela [28]; we refer interested readers to Hajłasz [21] and Björn-Björn [4] for detailed discussions on upper gradients.

The Newtonian space $N^{1, p}(X), 1 \leq p<\infty$, is defined as the collection of functions for which the norm

$$
\|u\|_{N^{1, p}(X)}:=\left(\int_{X} u^{p} d \mu+\inf _{g} \int_{X} g^{p} d \mu\right)^{1 / p}<\infty
$$

where the infimum is taken over all upper gradients of $u$. If $u \in N^{1, p}(X)$, then it has a minimal $p$-weak upper gradient $g_{u}$, which in our case is an upper gradient (since the empty set is the only curve family with zero $p$-modulus on a tree). The minimal upper gradient $g_{u}$ is unique up to measure zero, and is minimal in the sense that if $g \in L^{p}(X)$ is any upper gradient of $u$ then $g_{u} \leq g$ a.e. We refer the interested reader to Shanmugalingam [45, Corollary 3.7] $(p>1)$ and Hajłasz [21, Theorem 7.16] $(p \geq 1)$ for proofs of the existence and uniqueness of such a minimal upper gradient.

Our aim in this section is to establish the following 1-Poincaré inequality,

$$
f_{B}\left|u(y)-u_{B}\right| d \mu(y) \leq C r f_{B} g d \mu,
$$

where $u$ is integrable, $g$ is an arbitrary upper gradient of $u, r$ is the radius of $B$ and

$$
u_{E}:=f_{E} u d \mu:=\frac{1}{\mu(E)} \int_{E} u d \mu
$$

whenever $E$ is measurable and $0<\mu(E)<\infty$.

Lemma 4.1. Let $B=B(x, r) \subset X$ be a ball and let $z$ be as in Lemma 3.2. Then for every $u: B \rightarrow \mathbb{R}$ and every upper gradient $g$ of $u$ in $B$,

$$
\int_{B}|u(y)-u(z)| d \mu(y) \leq \int_{B} g(w) e^{(\beta-\varepsilon)|w|} \mu(\{y \in B: y \geq w\}) d \mu(w) .
$$

Proof. As $d_{X} s=e^{-\varepsilon|x|} d|x|=e^{(\beta-\varepsilon)|x|} d \mu(x)$, we obtain using the Fubini theorem 
that

$$
\begin{aligned}
\int_{B}|u(y)-u(z)| d \mu(y) & \leq \int_{B} \int_{[z, y]} g d_{X} s d \mu(y) \\
& =\int_{B} \int_{[z, y]} g(w) e^{(\beta-\varepsilon)|w|} d \mu(w) d \mu(y) \\
& =\int_{B} g(w) e^{(\beta-\varepsilon)|w|} \mu(\{y \in B: y \geq w\}) d \mu(w) .
\end{aligned}
$$

Theorem 4.2. The space $X=(X, d, \mu)$ supports a 1-Poincaré inequality.

For simplicity, the proof below will assume that $X$ is regular $K$-ary; to generalize the proof to nonregular trees one only needs to note the comment after Lemma 3.3.

Proof. Let $B=B(x, r)$ be a ball. We shall use the estimate from Lemma 4.1. Let us first estimate $|w|$ for $w \in B$. For $|w| \geq|x|$, we must have

$$
r>\int_{|x|}^{|w|} e^{-\varepsilon t} d t=\frac{1}{\varepsilon} e^{-\varepsilon|x|}\left(1-e^{\varepsilon(|x|-|w|)}\right)
$$

which yields

$$
|w|<|x|-\frac{1}{\varepsilon} \log \left(1-\varepsilon r e^{\varepsilon|x|}\right) .
$$

For $|w|<|x|$, this is trivially true. Now, we distinguish two cases.

(i) Assume first that $r \leq e^{-\varepsilon|x|} / 3 \varepsilon$, i.e. $\varepsilon r e^{\varepsilon|x|} \leq \frac{1}{3}$. A simple calculation using (4.2) shows that for all $w \in B$,

$$
e^{-\varepsilon|w|}>e^{-\varepsilon|x|}\left(1-\varepsilon r e^{\varepsilon|x|}\right) \geq \frac{2 e^{-\varepsilon|x|}}{3},
$$

and hence

Lemma 3.3 then implies that

$$
2 r \leq \frac{2 e^{-\varepsilon|x|}}{3 \varepsilon}<\frac{e^{-\varepsilon|w|}}{\varepsilon} .
$$

$$
\mu(\{y \in B: y \geq w\}) \leq \mu(F(w, 2 r)) \lesssim e^{(\varepsilon-\beta)|w|} r .
$$

Inserting this into Lemma 4.1 yields

$$
\int_{B}|u(y)-u(z)| d \mu(y) \lesssim r \int_{B} g(w) d \mu(w) .
$$

(ii) Assume instead that $r \geq e^{-\varepsilon|x|} / 3 \varepsilon$. Then

$$
\mu(\{y \in B: y \geq w\}) \leq \mu\left(F\left(w, e^{-\varepsilon|w|} / \varepsilon\right)\right) \lesssim e^{-\beta|w|},
$$

by Lemma 3.3. Inserting this into Lemma 4.1 yields

$$
\int_{B}|u(y)-u(z)| d \mu(y) \lesssim \int_{B} g(w) e^{-\varepsilon|w|} d \mu(w) \leq e^{-\varepsilon|z|} \int_{B} g(w) d \mu(w) .
$$

From (3.3) and the above choice of $r$ we have

$$
e^{-\varepsilon|z|} \leq e^{-\varepsilon|x|}\left(1+\varepsilon r e^{\varepsilon|x|}\right)=e^{-\varepsilon|x|}+\varepsilon r \leq 4 \varepsilon r .
$$

Together with (4.4), this proves (4.3) also for $r \geq e^{-\varepsilon|x|} / 3 \varepsilon$.

To finish the proof, observe that $u(z)$ in $(4.3)$ can by replaced by $u_{B}$ as follows

$$
f_{B}\left|u-u_{B}\right| d \mu \leq f_{B}|u(y)-u(z)| d \mu(y)+\left|u(z)-u_{B}\right| \leq 2 f_{B}|u(y)-u(z)| d \mu(y) .
$$


Corollary 4.3. The space $X=(X, d, \mu)$ supports a $(p, p)$-Poincaré inequality, i.e.

$$
f_{B}\left|u-u_{B}\right|^{p} d \mu \leq C r f_{B} g^{p} d \mu .
$$

That this follows from the 1-Poincaré inequality established in Theorem 4.2 is well known and a simple proof can be given along the lines of pp. 11-12 in Heinonen-Kilpeläinen-Martio [27] (alternatively one can e.g. appeal to Theorem 5.1 in Hajłasz-Koskela [22]).

\section{Hausdorff dimension of $\partial X$ and Besov functions on $\partial X$}

In Sections 5 and 6 we assume that $X$ is a regular $K$-ary tree, $K \geq 2$.

In this section we construct the boundary of the regular $K$-ary tree and show that it is Ahlfors regular with regularity exponent depending solely on $K$ and on the metric density exponent $\varepsilon$ of the tree. We then study a family of Besov spaces $B_{p, p}^{\theta}(\partial X)$ of functions on the boundary $\partial X$ of the tree, and prove that continuous functions are dense in these Besov spaces.

By the discussion in the previous two sections, we know that $X$ is a metric space equipped with a doubling measure supporting a 1-Poincaré inequality.

A tree is the quintessential Gromov hyperbolic space, and hence we can consider the visual boundary of the tree as in Bridson-Haefliger [12]. The discussion in BonkHeinonen-Koskela [8] tells us that this visual boundary is the same as the metric boundary of the tree equipped with the uniformizing metric $d_{X}$ given in (2.1). The focus of this section is to describe and study the properties of this boundary.

We define the boundary of a tree $X$, denoted $\partial X$, by completing $X$ with respect to the metric $d_{X}$. An equivalent construction of $\partial X$ is as follows. An element $\zeta$ in $\partial X$ is identified with an infinite geodesic in $X$ starting at the root 0 . If we denote the geodesic by concatenation of vertices, then

$$
\zeta=0 x_{1} x_{2} x_{3} \ldots,
$$

where $x_{i}$ is a vertex in $X$ at a distance $i$ from the root, and $x_{i+1}$ is a child of $x_{i}$. Given two points $\zeta, \xi \in \partial X$, the distance between them is the length (with respect to the metric $d_{X}$ ) of the infinite geodesic $[\zeta, \xi]$ between them. If this infinite geodesic is $k$ edges from the root 0 (counting each edge as having unit length) then by (2.1),

$$
d_{X}(\zeta, \xi)=2 \int_{k}^{\infty} e^{-\varepsilon t} d t=\frac{2}{\varepsilon} e^{-\varepsilon k}
$$

Following Bridson-Haefliger [12], the restriction of $d_{X}$ to $\partial X$ is called the visual metric on $\partial X$.

The metric $d_{X}$ is thus defined on $\bar{X}$ and we will consider balls with respect to this metric in $X, \bar{X}$ and $\partial X$. To avoid confusion, points in $X$ are denoted by Latin letters such as $x, y, z$ and $w$, while for points in $\partial X$ we use Greek letters such as $\zeta$, $\xi, \chi$ and $\omega$.

Balls in $X$ will thus be denoted $B(x, r)$, while $B(\zeta, r)$ stands for a ball in $\partial X$. Since $X$ and $\partial X$ are disjoint, this should not cause any confusion. For balls in $\bar{X}$ we write $B_{\bar{X}}(x, r)$ and $B_{\bar{X}}(\zeta, r)$, depending on whether the center lies in $X$ or $\partial X$.

Recall that a metric space $\left(Z, d_{Z}\right)$ is an ultrametric space if for each triple of points $x, y, z \in Z$ we have $d_{Z}(x, z) \leq \max \left\{d_{Z}(x, y), d_{Z}(y, z)\right\}$.

Lemma 5.1. The metric space $\left(\partial X, d_{X}\right)$ is an ultrametric space, and consequently, whenever $\zeta \in \partial X, r>0$, and $\xi \in B(\zeta, r)$, we have $B(\zeta, r)=B(\xi, r)$. 
Proof. Let $\zeta, \xi, \eta \in \partial X$. Let $k$ be the number of edges in the shortest curve connecting 0 to the infinite geodesic $[\zeta, \xi], k_{1}$ be the number of edges in the shortest curve connecting 0 to $[\zeta, \eta]$, and $k_{2}$ be the number of edges in the shortest curve connecting 0 to $[\xi, \eta]$. Then $k \geq \min \left\{k_{1}, k_{2}\right\}$, and so

$$
e^{-\varepsilon k} \leq \max \left\{e^{-\varepsilon k_{1}}, e^{-\varepsilon k_{2}}\right\}
$$

from which, together with (5.1), the ultra metric property follows. The latter part of the lemma is a direct consequence of the ultrametric property of $\partial X$.

Lemma 5.2. $\partial X$ is an Ahlfors $Q$-regular space with Hausdorff dimension

$$
Q=\frac{\log K}{\varepsilon}
$$

Proof. We equip $\partial X$ with the natural probability measure $\nu$ as in Falconer [16] by distributing the unit mass uniformly on $\partial X$. Let $x \in \partial X$ and $0<r \leq e^{\varepsilon} \operatorname{diam} \partial X=$ $e^{\varepsilon} \operatorname{diam} X=2 e^{\varepsilon} / \varepsilon$. Let $k \in \mathbb{Z}$ be such that

$$
\frac{2}{\varepsilon} e^{-\varepsilon k}<r \leq \frac{2}{\varepsilon} e^{-\varepsilon(k-1)}
$$

Then $\partial X$ is the union of $K^{k}$ disjoint open balls of radius $r$, each of which has, by definition, $\nu$-measure $K^{-k}$. Since any point of a ball can be used as a center we see that $\nu(B(\zeta, r))=K^{-k}$ for every $\zeta \in \partial X$, where

$$
k=k(r):=\left\lfloor 1+\frac{1}{\varepsilon} \log \frac{2}{\varepsilon r}\right\rfloor
$$

and so

$$
\nu(B(\zeta, r)) \simeq r^{Q}
$$

Since $\nu(\partial X)=1$, we see that (5.3) also holds even when $e^{\varepsilon} \operatorname{diam} X \leq r \leq 2 \operatorname{diam} X$ (but with different implicit constants). It also follows that

$$
\nu(A) \simeq \mathcal{H}^{Q}(A) \text { for all measurable sets } A \subset \partial X,
$$

where $\mathcal{H}^{Q}$ denotes the $Q$-dimensional Hausdorff measure. Thus $\nu$ is the normalized $Q$-dimensional Hausdorff measure on $\partial X$.

Example 5.3. The boundary $\partial X$ can be (up to a biLipschitz mapping) identified with a totally disconnected regular fractal set defined by $K$ similarities, each with contraction ratio $e^{-\varepsilon}$.

For example, $K=2$ and $\varepsilon=\log 3$ gives the usual ternary Cantor dust, while $K=4$ and $\varepsilon=\log 4$ gives the 1-dimensional Garnett-Ivanov set, which was the first example of a set in the plane with positive length but zero analytic capacity, see Garnett [18] and Ivanov [31, footnote on p. 346].

Letting $K=3$ and $\varepsilon=\log 3$ leads to the following 1-dimensional totally disconnected "Sierpiński dust": Split an equilateral triangle into 9 smaller congruent equilateral triangles and pick the three which contain the vertices of the original one. Repeat this construction for each of the chosen smaller triangles.

If we instead let $K=3$ and $\varepsilon=\log 2$ then the resulting fractal will have dimension $Q=(\log 3) /(\log 2)$. This dimension is the same as that of the Sierpiński gasket, but this Cantor set will be totally disconnected, i.e. the three subgenerations in the usual Sierpiński gasket have to be considered as having positive distance from each other. In fact, this fractal is just a snowflaked version of the above "Sierpiński dust" (with a new metric $|x-y|^{1 / Q}$ ). 
We now wish to find the connection between certain function spaces on $X$ and $\partial X$. Namely in $X$ we consider the Newtonian space $N^{1, p}(X)$, as defined in Section 4 . On the boundary, $\partial X$, we consider another space of functions, the Besov space. Let $f: \partial X \rightarrow \mathbb{R}$. Let $\nu$ denote the normalized $Q$-dimensional Hausdorff measure on $\partial X$. For $t>0$ and $p \geq 1$ we set

$$
E_{p}(f, t):=\left(\int_{\partial X} f_{B(\zeta, t)}|f(\zeta)-f(\xi)|^{p} d \nu(\xi) d \nu(\zeta)\right)^{1 / p}
$$

and for $\theta>0$ and $q \geq 1$,

$$
\|f\|_{B_{p, q}^{\theta}(\partial X)}:=\left(\int_{0}^{\infty}\left(\frac{E_{p}(f, t)}{t^{\theta}}\right)^{q} \frac{d t}{t}\right)^{1 / q} .
$$

The Besov space $B_{p, q}^{\theta}(\partial X)$ consists of all $f \in L^{p}(\partial X)$ for which this seminorm is finite. In this paper we only deal with the Besov spaces for which $q=p$, that is, the spaces $B_{p, p}^{\theta}(\partial X)$. The expression

$$
\|f\|_{\widetilde{B}_{p, p}^{\theta}(\partial X)}:=\|f\|_{L^{p}(\partial X)}+\|f\|_{B_{p, p}^{\theta}(\partial X)}
$$

is a norm on $B_{p, p}^{\theta}(\partial X)$.

The following lemma shows that the Besov seminorm (5.4) can equivalently be calculated as an infinite sum. We shall also see that in bounded spaces (as here) the integral in the definition of the Besov seminorm can be taken over a finite interval. We formulate these results for our situation, but they hold in any metric space, provided that the measure on it is doubling.

Lemma 5.4. Let $0<\sigma<1$ and $t_{n}=C \sigma^{n}, n \in \mathbb{Z}$. Then

$$
\|f\|_{B_{p, p}^{\theta}(\partial X)}^{p} \simeq \sum_{n=-\infty}^{\infty}\left(\frac{E_{p}\left(f, t_{n}\right)}{t_{n}^{\theta}}\right)^{p} .
$$

Furthermore,

$$
\|f\|_{B_{p, p}^{\theta}(\partial X)}^{p} \simeq \int_{\partial X} \int_{\partial X} \frac{|f(\zeta)-f(\xi)|^{p}}{d_{X}(\zeta, \xi)^{\theta p} \nu\left(B\left(\zeta, d_{X}(\zeta, \xi)\right)\right)} d \nu(\xi) d \nu(\zeta) .
$$

Proof. The doubling property for $\nu$ implies that for $t_{n+1} \leq t \leq t_{n}$,

$$
E_{p}\left(f, t_{n+1}\right) \lesssim E_{p}(f, t) \lesssim E_{p}\left(f, t_{n}\right)
$$

Hence

$$
\int_{t_{n+1}}^{t_{n}}\left(\frac{E_{p}(f, t)}{t^{\theta}}\right)^{p} \frac{d t}{t} \lesssim\left(\frac{E_{p}\left(f, t_{n}\right)}{t_{n}^{\theta}}\right)^{p} \int_{t_{n+1}}^{t_{n}} \frac{d t}{t} \simeq\left(\frac{E_{p}\left(f, t_{n}\right)}{t_{n}^{\theta}}\right)^{p}
$$

The lower bound in terms of $E_{p}\left(f, t_{n+1}\right)$ is obtained similarly and summing up over all $n \in \mathbb{Z}$ completes the proof of the first part. The second part follows directly from the computations in Gogatishvili-Koskela-Shanmugalingam [19, p. 226] or by combining the Ahlfors regularity of $\nu$ with (5.9) and (5.10) below (with $r_{n}$ replaced by 0$)$.

Remark 5.5. Note that if $\operatorname{diam} \partial X<t_{0}$, the sum in Lemma 5.4 can equivalently be taken over $n \geq 0$. Consequently, the integral in (5.4) can be taken over the finite interval $(0,2 \operatorname{diam} \partial X)$. 
It follows that

$$
B_{p, p}^{\theta_{2}}(\partial X) \subset B_{p, p}^{\theta_{1}}(\partial X) \text { if } 0<\theta_{1}<\theta_{2}
$$

The following example shows that the converse inclusion is false. This also directly yields that $B_{p, p}^{\theta}(\partial X)$ is nontrivial for all $\theta>0$. Note also that (5.5) and the Hölder inequality yield that for $1 \leq q<p$ and $0<\tau<\theta$,

$$
\|f\|_{B_{q, q}^{\tau}(\partial X)} \leq\|f\|_{B_{p, p}^{\theta}(\partial X)}\left(\int_{\partial X} \int_{\partial X} \frac{d_{X}(\zeta, \xi)^{(\theta-\tau) p q /(p-q)} d \nu(\xi) d \nu(\zeta)}{\nu\left(B\left(\zeta, d_{X}(\zeta, \xi)\right)\right)}\right)^{1 / q-1 / p}
$$

where the last integral converges since $\tau<\theta$. Thus

$$
B_{p, p}^{\theta}(\partial X) \subset B_{q, q}^{\tau}(\partial X) \quad \text { whenever } 1 \leq q \leq p \text { and } 0<\tau<\theta .
$$

Example 5.6. Let $\alpha>-Q / p$ with $\alpha \neq 0$. Fix $\zeta_{0} \in \partial X$ and set $f(\xi)=d_{X}\left(\xi, \zeta_{0}\right)^{\alpha}$. Further, let $0<t<e^{\varepsilon} \operatorname{diam} \partial X$ and let $k(t)$ be given by (5.2).

Let us first estimate $E_{p}(f, t)$ for $\alpha>0$. Let $\zeta \in B\left(\zeta_{0}, t\right)$ and $d=d_{X}\left(\zeta, \zeta_{0}\right)$. Note that if $d_{X}\left(\xi, \zeta_{0}\right)=d$, then $f(\zeta)=f(\xi)$. Thus

$$
\begin{aligned}
\int_{B\left(\zeta_{0}, t\right)}|f(\xi)-f(\zeta)|^{p} d \nu(\xi) & \leq \int_{B\left(\zeta_{0}, d\right)} f(\zeta)^{p} d \nu(\xi)+\int_{B\left(\zeta_{0}, t\right) \backslash B\left(\zeta_{0}, e^{\varepsilon} d\right)} f(\xi)^{p} d \nu(\xi) \\
& \leq \int_{B\left(\zeta_{0}, t\right) \backslash B\left(\zeta_{0}, d\right)} f(\xi)^{p} d \nu(\xi),
\end{aligned}
$$

since $\nu\left(B\left(\zeta_{0}, d\right)\right)=\nu\left(B\left(\zeta_{0}, e^{\varepsilon} d\right) \backslash B\left(\zeta_{0}, d\right)\right) /(K-1)$ and $f(\zeta)=f(\xi)$ for $\xi \epsilon$ $B\left(\zeta_{0}, e^{\varepsilon} d\right) \backslash B\left(\zeta_{0}, d\right)$. Hence, by summing over the shells around $\zeta_{0}$ we obtain that

$$
\begin{aligned}
f_{B\left(\zeta_{0}, t\right)}|f(\xi)-f(\zeta)|^{p} d \nu(\xi) & \leq f_{B\left(\zeta_{0}, t\right)} f(\xi)^{p} d \nu(\xi) \\
& \simeq K^{k(t)} \sum_{k=k(t)+1}^{\infty} e^{-\varepsilon k \alpha p} K^{-k} \simeq e^{-\varepsilon k(t) \alpha p} \simeq t^{\alpha p}
\end{aligned}
$$

Here we used the fact that for each integer $k>k(t)$ there are $K-1$ balls in $\partial X$ of radius $r_{k}=2 e^{-\varepsilon k} / \varepsilon$ and at distance $r_{k}$ from $\zeta_{0}$.

On the other hand, if $\zeta \in B\left(\zeta_{0}, t\right) \backslash B\left(\zeta_{0}, e^{-\varepsilon} t\right)$, then

$$
\begin{aligned}
f_{B\left(\zeta_{0}, t\right)}|f(\xi)-f(\zeta)|^{p} d \nu(\xi) & \gtrsim f_{B\left(\zeta_{0}, e^{-\varepsilon} t\right)}|f(\xi)-f(\zeta)|^{p} d \nu(\xi) \\
& \geq\left|\left(t e^{-2 \varepsilon}\right)^{\alpha}-\left(t e^{-\varepsilon}\right)^{\alpha}\right|^{p} \simeq t^{\alpha p}
\end{aligned}
$$

Since $B\left(\zeta_{0}, t\right)=B(\zeta, t)$, it thus follows that

$$
\int_{B\left(\zeta_{0}, t\right)} f_{B(\zeta, t)}|f(\xi)-f(\zeta)|^{p} d \nu(\xi) d \nu(\zeta) \simeq K^{-k(t)} t^{\alpha p} \simeq t^{Q} t^{\alpha p}
$$

if $\alpha>0$.

Let us similarly estimate for $-Q / p<\alpha<0$. If $\zeta \in B\left(\zeta_{0}, e^{-\varepsilon k} t\right) \backslash B\left(\zeta_{0}, e^{-\varepsilon(k+1)} t\right)$, where $k$ is a nonnegative integer, then after again summing over shells we get that

$$
\begin{aligned}
f_{B\left(\zeta_{0}, t\right)}|f(\xi)-f(\zeta)|^{p} d \nu(\xi) & \simeq \sum_{j=0}^{\infty} K^{-j}\left|e^{-\varepsilon(j+k(t)) \alpha}-e^{-\varepsilon(k+k(t)) \alpha}\right|^{p} \\
& \simeq e^{-\varepsilon k(t) \alpha p}\left(\sum_{j=0}^{k-1} K^{-j} e^{-\varepsilon k \alpha p}+\sum_{j=k+1}^{\infty} K^{-j} e^{-\varepsilon j \alpha p}\right) \\
& \simeq e^{-\varepsilon k(t) \alpha p}\left(e^{-\varepsilon k \alpha p}+e^{(-\varepsilon \alpha p-\log K) k}\right) \\
& \simeq t^{\alpha p} e^{-\varepsilon k \alpha p} .
\end{aligned}
$$


Thus

$$
\begin{aligned}
\int_{B\left(\zeta_{0}, t\right)} f_{B(\zeta, t)}|f(\xi)-f(\zeta)|^{p} d \nu(\xi) d \nu(\zeta) & \simeq t^{\alpha p} K^{-k(t)} \sum_{k=0}^{\infty} K^{-k} e^{-\varepsilon k \alpha p} \\
& \simeq t^{\alpha p} K^{-k(t)} \simeq t^{Q} t^{\alpha p}
\end{aligned}
$$

yielding the estimate (5.8) also for $\alpha<0$.

For $\zeta \in \partial X \backslash B\left(\zeta_{0}, t\right)$ we instead see that $f$ is constant within $B(\zeta, t)$, and thus

$$
\int_{\partial X \backslash B\left(\zeta_{0}, t\right)} f_{B(\zeta, t)}|f(\zeta)-f(\xi)|^{p} d \nu(\xi) d \nu(\zeta)=0 .
$$

Hence $E_{p}(f, t)^{p} \simeq t^{Q+\alpha p}$ and

$$
\|f\|_{B_{p, p}^{\theta}(\partial X)}^{p} \simeq \int_{0}^{2 e^{\varepsilon} / \varepsilon} \frac{t^{Q+\alpha p}}{t^{\theta p}} \frac{d t}{t}<\infty
$$

if and only if $\theta<\alpha+Q / p$.

Thus if $0<\theta_{1}<\theta_{2}$ we can choose $\alpha \neq 0$ such that $\theta_{1}-Q / p<\alpha<\theta_{2}-Q / p$ and obtain that $f \in B_{p, p}^{\theta_{1}}(\partial X) \backslash B_{p, p}^{\theta_{2}}(\partial X)$.

The functional analytic approach to Besov spaces in the classical Euclidean setting, using interpolation as in Bennett-Sharpley [3], as well as the approach to Besov spaces using atomic decompositions as in Triebel [47], immediately yield the density of continuous functions in the classical Besov spaces. Our definition of Besov spaces, equivalent to that of the interpolation approach of [3] (see GogatishviliKoskela-Shanmugalingam [19]) under the assumption that the underlying metric space has a doubling measure supporting a $p$-Poincaré inequality, does not on its own imply the density of continuous functions in the corresponding Besov space. Note that Cantor type sets do not support any Poincaré inequality for functionupper gradient pairs. However, we will next show that because of the ultrametric structure of the Cantor sets, continuous functions are indeed dense in the Besov space.

Proposition 5.7. The set of all Lipschitz continuous functions in $B_{p, p}^{\theta}(\partial X)$ is dense in $B_{p, p}^{\theta}(\partial X)$.

Proof. Let $u \in B_{p, p}^{\theta}(\partial X)$. We will approximate $u$ by continuous functions on $\partial X$ as follows. For $n \geq 1$, let $B_{n, i}, i=1,2, \ldots, K^{n}$, be the $K^{n}$ balls of radius $r_{n}=$ $2 e^{(1-n) \varepsilon} / \varepsilon$, whose union is $\partial X$. Note that all these balls have the same measure $\nu\left(B_{n, i}\right)=K^{-n} \simeq r_{n}^{Q}$. For each $n, i$ and $\xi \in B_{n, i}$ let

$$
u_{n}(\xi)=f_{B\left(\xi, r_{n}\right)} u d \nu=f_{B_{n, i}} u d \nu
$$

The functions $u_{n}$ are piecewise constant and Lipschitz continuous on $\partial X$, since $\left\{B_{n, i}\right\}_{i=1}^{K^{n}}$ form a pairwise disjoint clopen cover of $\partial X$. Let $v_{n}=u_{n}-u$. We shall show that $\left\|v_{n}\right\|_{B_{p, p}^{\theta}(\partial X)} \rightarrow 0$ as $n \rightarrow \infty$ (i.e. $r_{n} \rightarrow 0$ ). Note first that $u_{n}(\zeta)=u_{n}(\xi)$ whenever $d_{X}(\zeta, \xi)<r_{n}$. Hence $\left|v_{n}(\zeta)-v_{n}(\xi)\right|=|u(\zeta)-u(\xi)|$ for such $\zeta$ and $\xi$ and consequently,

$$
\begin{aligned}
I\left(r_{n}\right):= & \int_{0}^{r_{n}} \int_{\partial X} f_{B(\zeta, r)} \frac{\left|v_{n}(\zeta)-v_{n}(\xi)\right|^{p}}{r^{\theta p}} d \nu(\xi) d \nu(\zeta) \frac{d r}{r} \\
& =\int_{0}^{r_{n}} \int_{\partial X} f_{B(\zeta, r)} \frac{|u(\zeta)-u(\xi)|^{p}}{r^{\theta p}} d \nu(\xi) d \nu(\zeta) \frac{d r}{r} \rightarrow 0
\end{aligned}
$$


as $r_{n} \rightarrow 0$, by the finiteness of $\|u\|_{B_{p, p}^{\theta}(\partial X)}$. Next, by the Fubini theorem and the fact that $\nu(B(\zeta, r)) \simeq r^{Q}$ we see that

$$
\begin{aligned}
I I\left(r_{n}\right) & :=\int_{r_{n}}^{\infty} \int_{\partial X} f_{B(\zeta, r)} \frac{\left|v_{n}(\zeta)-v_{n}(\xi)\right|^{p}}{r^{\theta p}} d \nu(\xi) d \nu(\zeta) \frac{d r}{r} \\
& \simeq \int_{\partial X} \int_{\partial X}\left|v_{n}(\zeta)-v_{n}(\xi)\right|^{p} \int_{r_{n}}^{\infty} \frac{\mathbb{1}_{B(\zeta, r)}(\xi)}{r^{\theta p+Q}} \frac{d r}{r} d \nu(\xi) d \nu(\zeta)
\end{aligned}
$$

Since $\mathbb{1}_{B(\zeta, r)}(\xi) \neq 0$ if and only if $r>d_{X}(\zeta, \xi)$, the last integral becomes

$$
\int_{r_{n}}^{\infty} \frac{\mathbb{1}_{B(\zeta, r)}(\xi)}{r^{\theta p+Q}} \frac{d r}{r}=\frac{1}{\theta p+Q} \max \left\{r_{n}, d_{X}(\zeta, \xi)\right\}^{-\theta p-Q} \simeq \frac{1}{\left(r_{n}+d_{X}(\zeta, \xi)\right)^{\theta p+Q}} .
$$

Inserting this into (5.9) shows that

$$
I I\left(r_{n}\right) \simeq \int_{\partial X} \int_{\partial X} \frac{\left|v_{n}(\zeta)-v_{n}(\xi)\right|^{p}}{\left(r_{n}+d_{X}(\zeta, \xi)\right)^{\theta p+Q}} d \nu(\xi) d \nu(\zeta)
$$

Recall that $v_{n}=u_{n}-u$. To estimate the last integral we first note that by the Hölder and triangle inequalities,

$$
\begin{aligned}
\left|v_{n}(\zeta)-v_{n}(\xi)\right|^{p} & =\left|f_{B\left(\zeta, r_{n}\right)}(u(\chi)-u(\zeta)) d \nu(\chi)-f_{B\left(\xi, r_{n}\right)}(u(\omega)-u(\xi)) d \nu(\omega)\right|^{p} \\
& \leq 2^{p-1}\left(f_{B\left(\zeta, r_{n}\right)}|u(\chi)-u(\zeta)|^{p} d \nu(\chi)+f_{B\left(\xi, r_{n}\right)}|u(\omega)-u(\xi)|^{p} d \nu(\omega)\right) .
\end{aligned}
$$

Next, note that the roles of $\zeta$ and $\xi$ above and in (5.10) are symmetric, so that

$$
I I\left(r_{n}\right) \lesssim \int_{\partial X} \int_{\partial X} f_{B\left(\zeta, r_{n}\right)} \frac{|u(\chi)-u(\zeta)|^{p}}{\left(r_{n}+d_{X}(\zeta, \xi)\right)^{\theta p+Q}} d \nu(\chi) d \nu(\xi) d \nu(\zeta) .
$$

We next split the middle integral (with respect to $\xi$ ) into integrals over $B\left(\zeta, r_{n}\right)$ and $\partial X \backslash B\left(\zeta, r_{n}\right)$. The integral over $B\left(\zeta, r_{n}\right)$ is estimated as

$$
\begin{aligned}
& \int_{\partial X} \int_{B\left(\zeta, r_{n}\right)} f_{B\left(\zeta, r_{n}\right)} \frac{|u(\chi)-u(\zeta)|^{p}}{\left(r_{n}+d_{X}(\zeta, \xi)\right)^{\theta p+Q}} d \nu(\chi) d \nu(\xi) d \nu(\zeta) \\
& \leq \int_{\partial X} f_{B\left(\zeta, r_{n}\right)} \frac{|u(\chi)-u(\zeta)|^{p}}{r_{n}^{\theta p}} d \nu(\chi) d \nu(\zeta)
\end{aligned}
$$

while the integral over $\partial X \backslash B\left(\zeta, r_{n}\right)$ is split into integrals over the $K^{n}-1$ balls $B_{n, i}$ with radii $r_{n}$. Note that $\nu\left(B_{n, i}\right)=\nu\left(B\left(\zeta, r_{n}\right)\right)=K^{-n} \simeq r_{n}^{Q}$ and for each $j=0, \ldots, n-1$ there are $(K-1) K^{j}$ such balls $B_{n, i}$ which have distance $e^{j \varepsilon} r_{n}$ to $B\left(\zeta, r_{n}\right)$, i.e. $d_{X}(\zeta, \xi)=e^{j \varepsilon} r_{n}$ for $\xi \in B_{n, i}$. As $\xi$ only appears in the denominator $\left(r_{n}+d_{X}(\zeta, \xi)\right)^{\theta p+Q}$, summing up over all these balls $B_{n, i}$ gives

$$
\begin{aligned}
& \int_{\partial X} \int_{\partial X \backslash B\left(\zeta, r_{n}\right)} f_{B\left(\zeta, r_{n}\right)} \frac{|u(\chi)-u(\zeta)|^{p}}{\left(r_{n}+d_{X}(\zeta, \xi)\right)^{\theta p+Q}} d \nu(\chi) d \nu(\xi) d \nu(\zeta) \\
& \quad \leq \int_{\partial X} f_{B\left(\zeta, r_{n}\right)}|u(\chi)-u(\zeta)|^{p} \sum_{j=0}^{n-1} \frac{(K-1) K^{j} \nu\left(B\left(\zeta, r_{n}\right)\right)}{\left(e^{j \varepsilon} r_{n}\right)^{\theta p+Q}} d \nu(\chi) d \nu(\zeta) .
\end{aligned}
$$

Since $K / e^{\varepsilon(\theta p+Q)}=e^{-\varepsilon \theta p}<1$, the sum is majorized by $C \nu\left(B\left(\zeta, r_{n}\right)\right) / r_{n}^{\theta p+Q} \simeq r_{n}^{-\theta p}$. Inserting this into (5.12) together with (5.11) shows that

$$
I I\left(r_{n}\right) \lesssim \int_{\partial X} f_{B\left(\zeta, r_{n}\right)} \frac{|u(\chi)-u(\zeta)|^{p}}{r_{n}^{\theta p}} d \nu(\chi) d \nu(\zeta)=\left(\frac{E_{p}\left(u, r_{n}\right)}{r_{n}^{\theta}}\right)^{p}
$$


From Lemma 5.4 and the finiteness of $\|u\|_{B_{p, p}^{\theta}(\partial X)}$ we now easily conclude that $I I\left(r_{n}\right) \rightarrow 0$ as $r_{n} \rightarrow 0$, and hence $\left\|u_{n}-u\right\|_{B_{p, p}^{\theta}(\partial X)} \rightarrow 0$ as $n \rightarrow \infty$. Since

$$
\begin{aligned}
\left\|u_{n}-u\right\|_{L^{p}(\partial X)}^{p} & =\int_{\partial X}\left|u_{n}(\zeta)-u(\zeta)\right|^{p} d \nu(\zeta) \\
& \leq \int_{\partial X} f_{B\left(\zeta, r_{n}\right)}|u(\chi)-u(\zeta)|^{p} d \nu(\chi) d \nu(\zeta),
\end{aligned}
$$

we also see that $\left\|u_{n}-u\right\|_{L^{p}(\partial X)}^{p} \rightarrow 0$ as $r_{n} \rightarrow 0$.

\section{Traces of Newtonian functions on regular trees}

Recall that in Sections 5 and 6 we assume that $X$ is a regular $K$-ary tree, $K \geq 2$.

In this section, we consider conditions under which Newtonian functions on $X$ have a Besov trace on $\partial X$, and when we may extend Besov functions on $\partial X$ to Newtonian functions on $X$. We shall see that the obtained results are sharp and in Theorem 6.5 we give a precise trace result.

Proposition 6.1. Let $X$ be a regular $K$-ary tree with the metric $d_{X}$ defined by the exponential weight as in (2.1) with $\varepsilon>0$ and the measure $\mu$ defined by the exponential weight with $\beta>\log K$, and let $p \geq 1$. Then for every $\theta$ satisfying

$$
0<\theta \leq 1-\frac{\beta-\log K}{p \varepsilon}
$$

there is a bounded linear trace operator $\operatorname{Tr}: N^{1, p}(X) \rightarrow B_{p, p}^{\theta}(\partial X)$ such that for $f \in N^{1, p}(X)$,

$$
\|\operatorname{Tr} f\|_{L^{p}(\partial X)} \leq|f(0)|+C\left\|g_{f}\right\|_{L^{p}(X)} \quad \text { and } \quad\|\operatorname{Tr} f\|_{B_{p, p}^{\theta}(\partial X)} \lesssim\left\|g_{f}\right\|_{L^{p}(X)} .
$$

In particular, $\|\operatorname{Tr} f\|_{\widetilde{B}_{p, p}^{\theta}(\partial X)} \lesssim\|f\|_{N^{1, p}(X)}$. Furthermore, for Lipschitz functions $f: X \rightarrow \mathbb{R}$ we have that $\operatorname{Tr} f=\left.f\right|_{\partial X}$, where the continuous extension of $f$ to $\bar{X}$ is also denoted by $f$.

Examples 6.2 and 6.3 below show that Proposition 6.1 is sharp.

When $p>1,0<\theta<1-(\beta-\log K) / p \varepsilon$ and $1 \leq \beta / \varepsilon<Q+1$, the above result can be deduced from Theorem 6.5 in Gogatishvili-Koskela-Shanmugalingam [19], which deals with general metric spaces supporting a Poincaré inequality, and their Ahlfors regular subsets. The borderline case $\theta=1-(\beta-\log K) / p \varepsilon$ can however not be obtained from [19]. To apply Theorem 6.5 from [19] note first that the parameter $\gamma$ is, in our setting, given by $\gamma=Q=(\log K) / \varepsilon$, the Hausdorff dimension of $\partial X$. We then find $q<p$ such that $p<s q /(s-Q)(Q+1)$, which is only possible if $s<Q+1$. Furthermore, let $\alpha$ be such that $\theta<\alpha<1-(s-Q) / p$. If we choose $q$ above sufficiently close to $p$ we can then find $\lambda<1-\alpha$ satisfying the remaining requirements in Theorem 6.5 in [19]. After noting that $p^{*}>p$, our result (in the case mentioned above) follows from (5.7).

For $\beta<\varepsilon,[19]$ gives less sharp exponents then ours, and for $p=1$ and $Q+1 \leq$ $s:=\max \{1, \beta / \varepsilon\}<Q+p$ one cannot obtain any embedding from [19]. Our range $\beta / \varepsilon<Q+p$ is an improvement on [19] in our setting, and is sharp by Example 6.2.

Note that in the classical Euclidean setting, if a set $K \subset \mathbb{R}^{n}$ is Ahlfors $d$-regular and compact, then the trace space (on $K$ ) of the classical Sobolev space $W^{1, p}\left(\mathbb{R}^{n}\right)$ is the Besov space $B_{p, p}^{\tau}(K)$ with $\tau=1-(n-d) / p$. This result is due to JonssonWallin [33], [34] and meshes well with our trace result, since the dimension of the (ambient) tree is $s=\max \{1, \beta / \varepsilon\}$ and the dimension of the boundary is $(\log K) / \varepsilon$. 
In Bourdon-Pajot [11, Théorème 0.1], the Besov space $B_{p, p}^{Q / p}$ on a uniformly perfect Ahlfors $Q$-regular metric space $Z$ is identified with the $l^{p}$-cohomology of the conformal gauge of $Z$. Their arguments utilize Theorem 9.8 from Bonk-HeinonenKoskela [8] and thus apply provided that $Z$ is a continuum. In our setting, $\partial X$ is a totally disconnected set. Its cohomology corresponds to the class of functions $f: V \rightarrow \mathbb{R}$, defined on the set $V$ of vertices of $X$, whose differential $d f \in l^{p}(E)$ (where $E$ is the set of edges of $X$ ). Comparing $d f$ with (6.7) shows that for every edge $[x, y] \in E$, we have $|d f| \simeq e^{\varepsilon|x|} g_{f}$. Hence $d f \in l^{p}(E)$ if and only if $g_{f} \in L^{p}(X, \mu)$, where $\mu$ is given by (3.1) with $\beta=p \varepsilon$. Note that with this choice of parameters we have $\theta=Q / p=1-(\beta / \varepsilon-Q) / p$. Thus, in our setting of regular trees, a result corresponding to Théorème 0.1 in [11] follows from Theorem 6.5 .

A direct consequence of Proposition 6.1 and Example 6.2 below is that for $p>1$ there is a bounded linear trace operator $\operatorname{Tr}: N^{1, p}(X) \rightarrow L^{p}(\partial X)$ if and only if $p>(\beta-\log K) / \varepsilon$. For $p=1$ the same is true except that we do not know if there is a bounded linear trace operator if $p=1=(\beta-\log K) / \varepsilon$.

Example 6.2. Let $f$ be the continuous function on $X$ given by $f(x)=\log (|x|+1)$. Note that the function $g(x)=e^{\varepsilon|x|} /(|x|+1)$ is an upper gradient of $f$ on $X$ with respect to the metric $d_{X}$, and so

$$
\int_{X} g_{f}^{p} d \mu \simeq \sum_{k=0}^{\infty} \frac{e^{p \varepsilon k}}{(k+1)^{p}} K^{k} e^{-\beta k}=\sum_{k=0}^{\infty} \frac{e^{(p \varepsilon-\beta+\log K) k}}{(k+1)^{p}}
$$

which is finite if and only if either $p<(\beta-\log K) / \varepsilon$ or $p=(\beta-\log K) / \varepsilon>1$. In this case we also see that $f \in L^{p}(X)$, by the $(p, p)$-Poincaré inequality in Corollary 4.3, and thus $f \in N^{1, p}(X)$.

On the other hand, $f(x) \rightarrow \infty$ as $x \rightarrow \partial X$. So the only reasonable trace $f$ can leave at $\partial X$ is the function which is $\infty$ everywhere, which does not belong to any Besov or Lebesgue space. The requirement

$$
p>\frac{\beta-\log K}{\varepsilon}
$$

is therefore necessary to be able to have any trace result, with the possible exception of a trace result also when $p=1=(\beta-\log K) / \varepsilon$.

Example 6.3. In this example we shall show that the range in (6.1) is sharp. Assume that

$$
\theta>1-\frac{\beta-\log K}{p \varepsilon} .
$$

Then we can find $\gamma$ such that $\max \{\varepsilon(1-\theta), 0\}<\gamma<\min \{\varepsilon,(\beta-\log K) / p\}$.

Let $f$ be the continuous function on $X$ defined as follows: We set $f(0)=0$, and for each vertex $x$ we choose exactly one child $c(x)$ of $x$ and set (recursively) $f(c(x))=f(x)+e^{(\gamma-\varepsilon)|x|}$, while for all other children $y$ of $x$ we set $f(y)=f(x)$. Finally we require $f$ to be linear on each of the edges (with respect to the $d_{X}$ metric), i.e.,

$$
f(t)=f(x)+(f(w)-f(x)) \frac{d_{X}(t, x)}{d_{X}(w, x)} \quad \text { for } t \in[x, w],
$$

for any child $w$ of the vertex $x$.

It follows that the function $g(t)=\varepsilon e^{\gamma|x|} /\left(1-e^{-\varepsilon}\right)$ when $t \in[x, c(x)]$ for some vertex $x$, and $g(t)=0$ for all other values of $t$, is an upper gradient of $f$. Hence, in a similar way to Example 6.2 we see that $f \in N^{1, p}(X)$ (since $p \gamma<\beta-\log K$ ) and that $f$ is a bounded continuous function (because $\gamma<\varepsilon$ ) which has a continuous extension to $\partial X$ (also denoted by $f)$. 
For $r<e^{\varepsilon} \operatorname{diam} X$, let $k(r)$ be as in (5.2). Let $B_{i}$ be any of the $K^{k(r)}$ pairwise disjoint balls with radius $r$, whose union is $\partial X$, and let $z$ be the largest common ancestor of all the points in $B_{i}$. Note that $|z|=k(r)$. We define a bijection $H$ between the subtree $\{x \in X: x \geq z\}$ and $X$ inductively as follows: $H(z)=0$ and for each vertex $x \geq z$, the children of $x$ are mapped to the children of $H(x)$ in such a way that $H(c(x))=c(H(x))$. Note that this bijection extends to a bijection between $B_{i}$ and $\partial X$, and that for $\xi \in B_{i}$,

$$
d \nu(\xi)=K^{-k(r)} d \nu(H(\xi))
$$

Moreover, if $y$ is a child of some $x \geq z$, then

$$
f(y)-f(x)=e^{(\gamma-\varepsilon) k(r)}(f(H(x))-f(H(y))) .
$$

Thus, for $\zeta, \xi \in B_{i}$, we obtain by continuity that

$$
f(\zeta)-f(\xi)=e^{(\gamma-\varepsilon) k(r)}(f(H(\zeta))-f(H(\xi))) .
$$

Together with (6.2) this yields

$$
f_{B_{i}} f_{B_{i}}|f(\zeta)-f(\xi)|^{p} d \nu(\xi) d \nu(\zeta)=e^{p(\gamma-\varepsilon) k(r)} f_{\partial X} f_{\partial X}|f(\zeta)-f(\xi)|^{p} d \nu(\xi) d \nu(\zeta),
$$

where the last double integral is clearly positive, finite and independent of $r$ and $i$. Multiplying the last identity by $\nu\left(B_{i}\right)=K^{-k(r)}$ and summing over $i=1, \ldots, K^{k(r)}$ gives, since $B(\zeta, r)=B_{i}$ for $\zeta \in B_{i}$,

$$
E_{p}(f, r)^{p}=\sum_{i=1}^{K^{k(r)}} \int_{B_{i}} f_{B_{i}}|f(\zeta)-f(\xi)|^{p} d \nu(\xi) d \nu(\zeta) \simeq e^{p(\gamma-\varepsilon) k(r)} \simeq r^{p(\varepsilon-\gamma) / \varepsilon}
$$

Hence, by Remark 5.5,

$$
\left\|\left.f\right|_{\partial X}\right\|_{B_{p, p}^{\theta}(\partial X)}^{p} \simeq \int_{0}^{2 \operatorname{diam} X} \frac{t^{p(\varepsilon-\gamma) / \varepsilon}}{t^{\theta p}} \frac{d t}{t}=\infty
$$

because $\theta \geq 1-\gamma / \varepsilon$. Thus $\left.f\right|_{\partial \Omega} \notin B_{p, p}^{\theta}(\partial X)$.

Proof of Proposition 6.1. Let $f \in N^{1, p}(X)$. We shall first show that for $\nu$-a.e. $\zeta \in \partial X$, the limit

$$
\tilde{f}(\zeta)=\lim _{[0, \zeta) \ni x \rightarrow \zeta} f(x)
$$

taken along the geodesic ray $[0, \zeta)$, exists and defines the trace $\operatorname{Tr} f:=\tilde{f}: \partial X \rightarrow \mathbb{R}$, with norm estimates. (Note that if $f$ is Lipschitz, then $\tilde{f}(\zeta)=\lim _{x \rightarrow \zeta} f(x)$ for every $\zeta \in \partial X$

To this end, let $\zeta \in \partial X$ be arbitrary and let $x_{j}=x_{j}(\zeta)$ be the ancestor of $\zeta$ with $\left|x_{j}\right|=j$. Set $r_{j}=2 e^{-j \varepsilon} / \varepsilon$. Recall that on the edge $\left[x_{j}, x_{j+1}\right]$ we have $d s \simeq e^{(\beta-\varepsilon) j} d \mu \simeq r_{j}^{1-\beta / \varepsilon} d \mu$. Fix $n \in \mathbb{N}$ and let $m \geq n$ be arbitrary. Then

$$
\begin{aligned}
\left|f\left(x_{m}\right)-f\left(x_{n}\right)\right| & \leq \sum_{j=n}^{m-1}\left|f\left(x_{j+1}\right)-f\left(x_{j}\right)\right| \\
& \leq \sum_{j=n}^{\infty} \int_{\left[x_{j}, x_{j+1}\right]} g_{f} d s \simeq \sum_{j=n}^{\infty} r_{j}^{1-\beta / \varepsilon} \int_{\left[x_{j}, x_{j+1}\right]} g_{f} d \mu .
\end{aligned}
$$

We shall now show that, independently of $m$, this tends to zero as $n \rightarrow \infty$, for $\nu$-a.e. $\zeta \in \partial X$. Thus, the sequence $\left\{f\left(x_{n}\right)\right\}_{n=0}^{\infty}$ is a Cauchy sequence, and has a limit as $n \rightarrow \infty$, for $\nu$-a.e. $\zeta \in \partial X$. 
Choose $0<\kappa<\theta p$ and insert $r_{j}^{\kappa / p} r_{j}^{-\kappa / p}$ into the above sum. If $p>1$, then Hölder's inequality applied first to the integral and then to the sum, together with the estimate $\mu\left(\left[x_{j}, x_{j+1}\right]\right) \simeq r_{j}^{\beta / \varepsilon}$, implies that

$$
\begin{aligned}
\sum_{j=n}^{\infty} r_{j}^{1-\beta / \varepsilon} \int_{\left[x_{j}, x_{j+1}\right]} g_{f} d \mu & \leq \sum_{j=n}^{\infty} r_{j}^{\kappa / p} r_{j}^{1-\beta / \varepsilon p-\kappa / p}\left(\int_{\left[x_{j}, x_{j+1}\right]} g_{f}^{p} d \mu\right)^{1 / p} \\
& \lesssim r_{n}^{\kappa / p}\left(\sum_{j=n}^{\infty} r_{j}^{p-\beta / \varepsilon-\kappa} \int_{\left[x_{j}, x_{j+1}\right]} g_{f}^{p} d \mu\right)^{1 / p}
\end{aligned}
$$

since $r_{j}=r_{n} e^{(n-j) \varepsilon}$, which gives the convergent sum $\sum_{j=n}^{\infty} r_{j}^{\kappa /(p-1)} \simeq r_{n}^{\kappa /(p-1)}$. (For $p=1$ the estimate is simpler and Hölder's inequality is not needed.) It follows that

$$
\left|f\left(x_{m}(\zeta)\right)-f\left(x_{n}(\zeta)\right)\right|^{p} \lesssim r_{n}^{\kappa} \sum_{j=n}^{\infty} r_{j}^{p-\beta / \varepsilon-\kappa} \int_{\left[x_{j}, x_{j+1}\right]} g_{f}^{p} d \mu .
$$

Integrating over all $\zeta \in \partial X$ we obtain by means of Fubini's theorem,

$$
\begin{aligned}
\int_{\partial X} \mid f( & \left.x_{m}(\zeta)\right)-\left.f\left(x_{n}(\zeta)\right)\right|^{p} d \nu(\zeta) \\
& \lesssim r_{n}^{\kappa} \int_{\partial X} \sum_{j=n}^{\infty} r_{j}^{p-\beta / \varepsilon-\kappa} \int_{\left[x_{j}, x_{j+1}\right]} g_{f}^{p} d \mu d \nu(\zeta) \\
& =r_{n}^{\kappa} \int_{X} g_{f}(x)^{p} \int_{\partial X} \sum_{j=n}^{\infty} r_{j}^{p-\beta / \varepsilon-\kappa} \mathbb{1}_{\left[x_{j}, x_{j+1}\right]}(x) d \nu(\zeta) d \mu(x)
\end{aligned}
$$

Note that $\mathbb{1}_{\left[x_{j}, x_{j+1}\right]}(x)$ is nonzero only if $j \leq|x| \leq j+1$ and $x<\zeta$. Thus, the last estimate can be written as

$$
\int_{\partial X}\left|f\left(x_{m}(\zeta)\right)-f\left(x_{n}(\zeta)\right)\right|^{p} d \nu(\zeta) \lesssim r_{n}^{\kappa} \int_{X} g_{f}(x)^{p} r_{j(x)}^{p-\beta / \varepsilon-\kappa} \nu(E(x)) d \mu(x),
$$

where $E(x)=\{\zeta \in \partial X: \zeta>x\}$ and $j(x)$ is the largest integer such that $j(x) \leq|x|$. Since $\nu(E(x)) \lesssim r_{j(x)}^{Q}$ and $p-\beta / \varepsilon-\kappa+Q>0$, we obtain that

$$
\begin{aligned}
\int_{\partial X}\left|f\left(x_{m}(\zeta)\right)-f\left(x_{n}(\zeta)\right)\right|^{p} d \nu(\zeta) & \lesssim r_{n}^{\kappa} \int_{X} g_{f}(x)^{p} r_{j(x)}^{p-\beta / \varepsilon-\kappa+Q} d \mu(x) \\
& \lesssim r_{n}^{\kappa} \int_{X} g_{f}^{p} d \mu \rightarrow 0, \quad \text { as } n \rightarrow \infty
\end{aligned}
$$

Hence, the sequence $\left\{f\left(x_{n}\right)\right\}_{n=0}^{\infty}$ is a Cauchy sequence for $\nu$-a.e. $\zeta \in \partial X$, and has a limit as $n \rightarrow \infty$. This also shows (by letting $n=0$ and $f\left(x_{m}(\zeta)\right) \rightarrow \tilde{f}(\zeta)$ ) that

$$
\int_{\partial X}|\tilde{f}(\zeta)-f(0)|^{p} d \nu(\zeta) \lesssim \int_{X} g_{f}^{p} d \mu
$$

and thus $\|\tilde{f}\|_{L^{p}(\partial X)} \leq|f(0)|+C\left\|g_{f}\right\|_{L^{p}(X)}$.

To estimate $\|\tilde{f}\|_{B_{p, p}^{\theta}(\partial X)}$, we let $m \rightarrow \infty$ in (6.4) to obtain for $\nu$-a.e. $\zeta \in \partial X$,

$$
\left|\tilde{f}(\zeta)-f\left(x_{n}\right)\right|^{p} \lesssim r_{n}^{\kappa} \sum_{j=n}^{\infty} r_{j}^{p-\beta / \varepsilon-\kappa} \int_{\left[x_{j}, x_{j+1}\right]} g_{f}^{p} d \mu .
$$

A similar estimate holds for $\nu$-a.e. $\xi \in \partial X$ such that $d_{X}(\zeta, \xi)=r_{n}$, with the $x_{j}$ 's replaced by the ancestors $y_{j}$ of $\xi$. Note that $x_{n}=y_{n}$ for such $\xi$. It follows that

$$
|\tilde{f}(\xi)-\tilde{f}(\zeta)|^{p} \lesssim r_{n}^{\kappa} \sum_{j=n}^{\infty} r_{j}^{p-\beta / \varepsilon-\kappa}\left(\int_{\left[x_{j}, x_{j+1}\right]} g_{f}^{p} d \mu+\int_{\left[y_{j}, y_{j+1}\right]} g_{f}^{p} d \mu\right),
$$


where $n=n(\zeta, \xi) \approx-\log \left(\varepsilon d_{X}(\zeta, \xi) / 2\right)$ denotes the level of the largest common ancestor of $\zeta$ and $\xi$. Inserting this into (5.5) yields

$$
\begin{aligned}
\|\tilde{f}\|_{B_{p, p}^{\theta}(\partial X)}^{p} & \int_{\partial X} \int_{\partial X} \frac{r_{n}^{\kappa}}{d_{X}(\zeta, \xi)^{\theta p+Q}} \\
& \times \sum_{j=n}^{\infty} r_{j}^{p-\beta / \varepsilon-\kappa}\left(\int_{\left[x_{j}, x_{j+1}\right]} g_{f}^{p} d \mu+\int_{\left[y_{j}, y_{j+1}\right]} g_{f}^{p} d \mu\right) d \nu(\xi) d \nu(\zeta)
\end{aligned}
$$

where again $n=n(\zeta, \xi)$ depends on $\zeta$ and $\xi$. Because the roles of $\zeta$ and $\xi$ in the last formula are symmetric, it suffices to estimate the expression with the integral over $\left[x_{j}, x_{j+1}\right]$. By writing $\partial X=\bigcup_{n=0}^{\infty} A_{n}$, where $A_{n}=\left\{\xi \in \partial X: d_{X}(\xi, \zeta)=r_{n}\right\}$, together with the estimate $\nu\left(A_{n}\right) \lesssim r_{n}^{Q}$, this leads to

$$
\begin{aligned}
\|\tilde{f}\|_{B_{p, p}^{\theta}(\partial X)}^{p} & \lesssim \int_{\partial X} \sum_{n=0}^{\infty} r_{n}^{-\theta p-Q+\kappa} \int_{A_{n}} \sum_{j=n}^{\infty} r_{j}^{p-\beta / \varepsilon-\kappa} \int_{\left[x_{j}, x_{j+1}\right]} g_{f}^{p} d \mu d \nu(\xi) d \nu(\zeta) \\
& \lesssim \int_{\partial X} \sum_{n=0}^{\infty} r_{n}^{-\theta p+\kappa} \sum_{j=n}^{\infty} r_{j}^{p-\beta / \varepsilon-\kappa} \int_{X} g_{f}(x)^{p} \mathbb{1}_{\left[x_{j}, x_{j+1}\right]}(x) d \mu(x) d \nu(\zeta),
\end{aligned}
$$

where the edge $\left[x_{j}, x_{j+1}\right]$ belongs to the geodesic ray connecting the root 0 to $\zeta$. Note that $\mathbb{1}_{\left[x_{j}, x_{j+1}\right]}(x)$ is nonzero only if $n \leq j \leq|x| \leq j+1$ and $x<\zeta$. Thus, the last estimate can be written as

$$
\begin{aligned}
& \|\tilde{f}\|_{B_{p, p}^{\theta}(\partial X)}^{p} \\
& \quad \lesssim \int_{\partial X} \sum_{n=0}^{\infty} r_{n}^{-\theta p+\kappa} \int_{X} g_{f}(x)^{p} r_{j(x)}^{p-\beta / \varepsilon-\kappa} \mathbb{1}_{\{z \in X: \zeta>z\}}(x) \mathbb{1}_{\{z \in X:|z| \geq n\}}(x) d \mu(x) d \nu(\zeta) \\
& \quad \lesssim \int_{\partial X} \int_{X} g_{f}(x)^{p} r_{j(x)}^{p-\beta / \varepsilon-\kappa} \mathbb{1}_{\{z \in X: \zeta>z\}}(x) \sum_{n=0}^{j(x)} r_{n}^{-\theta p+\kappa} d \mu(x) d \nu(\zeta),
\end{aligned}
$$

where $j(x)$ is the largest integer such that $j(x) \leq|x|$. Using the Fubini theorem we then get

$$
\|\tilde{f}\|_{B_{p, p}^{\theta}(\partial X)}^{p} \lesssim \int_{X} g_{f}(x)^{p} r_{j(x)}^{p-\beta / \varepsilon-\kappa} \nu(E(x)) \sum_{n=0}^{j(x)} r_{n}^{-\theta p+\kappa} d \mu(x)
$$

The last sum is comparable to $r_{j(x)}^{-\theta p+\kappa}$, by the choice $\kappa<\theta p$. Since $\nu(E(x)) \lesssim r_{j(x)}^{Q}$ and $p-\beta / \varepsilon-\theta p+Q \geq 0$, we finally obtain that

$$
\|\tilde{f}\|_{B_{p, p}^{\theta}(\partial X)}^{p} \lesssim \int_{X} g_{f}(x)^{p} r_{j(x)}^{p-\beta / \varepsilon-\theta p+Q} d \mu(x) \lesssim \int_{X} g_{f}^{p} d \mu .
$$

We next show that Besov functions on the boundary Cantor set can be extended to Newtonian functions on the regular $K$-ary tree.

Proposition 6.4. Let $X$ be a regular $K$-ary tree with the metric $d_{X}$ defined by the exponential weight as in (2.1) with $\varepsilon>0$ and the measure $\mu$ defined by the exponential weight with $\beta>\log K$, and let $p \geq 1$. Suppose that

$$
\theta \geq 1-\frac{\beta-\log K}{p \varepsilon} \text { and } \theta>0 .
$$

Then there is a bounded linear extension operator

$$
\text { Ext }: B_{p, p}^{\theta}(\partial X) \longrightarrow N^{1, p}(X),
$$


such that for $u \in B_{p, p}^{\theta}(\partial X)$, we have $\operatorname{Tr}(\operatorname{Ext} u)=u \nu$-a.e., where $\operatorname{Tr}$ is the trace operator constructed in (6.3). Furthermore, for $\nu$-a.e. $\zeta \in \partial X$ and a geodesic $\gamma$ in $X$ terminating at $\zeta$, we have $\lim _{t \rightarrow \infty} \operatorname{Ext} u(\gamma(t))=u(\zeta)$. Moreover, with $\tilde{u}=$ Ext $u$, we have

$$
\begin{aligned}
\left\|g_{\tilde{u}}\right\|_{L^{p}(X)} & \lesssim\|u\|_{B_{p, p}^{\theta}(\partial X)}, \\
\|\tilde{u}\|_{N^{1, p}(X)} & \lesssim\|u\|_{L^{p}(\partial X)}+\|u\|_{B_{p, p}^{\theta}(\partial X)}=\|u\|_{\widetilde{B}_{p, p}^{\theta}(\partial X)}
\end{aligned}
$$

In view of Proposition 6.1, the range in (6.5) is sharp, cf. Theorem 6.5. Note also that if $u \in B_{p, p}^{\theta}(\partial X)$ is continuous then we have $\operatorname{Tr}(\operatorname{Ext} u)=u$ everywhere.

Proof. Let $u \in B_{p, p}^{\theta}(\partial X)$. For $x \in X$ with $|x|=n \in \mathbb{N}:=\{0,1,2, \ldots\}$ let

$$
\tilde{u}(x)=f_{B\left(\zeta, r_{n}\right)} u d \nu
$$

where $r_{n}=2 e^{(1-n) \varepsilon} / \varepsilon$ is as in the proof of Proposition 5.7 and $\zeta \in \partial X$ is any descendant of $x$. Observe that $B\left(\zeta, r_{n}\right)$ consists of all the points in $\partial X$ that have $x$ as an ancestor, that is, the geodesics connecting the root 0 to these points pass through $x$. Note that $\tilde{u}(x)=u_{n}(\zeta)$, where $u_{n}$ is the piecewise constant (and continuous) approximation of $u$ from Proposition 5.7. Moreover, (6.3) and (6.6) imply that $\operatorname{Tr} \tilde{u}(\zeta)=u(\zeta)$ whenever $\zeta \in \partial X$ is a Lebesgue point of $u$.

If $y$ is a child of $x$, extend $\tilde{u}$ to the edge $[x, y]$ as follows: First, we choose $\zeta \in \partial X$ so that $\zeta$ is a descendant of $y$ as well. We can do this because the ultrametric property of $\partial X$ tells us that every point in the ball $B\left(\zeta, r_{n}\right)$ is a center of this ball, see Lemma 5.1. For each $t \in[x, y]$ set

$$
g_{\tilde{u}}(t)=\frac{|\tilde{u}(x)-\tilde{u}(y)|}{d_{X}(x, y)}=\frac{\varepsilon\left|u_{n}(\zeta)-u_{n+1}(\zeta)\right|}{\left(1-e^{-\varepsilon}\right) e^{-\varepsilon n}}
$$

and $\tilde{u}(t)=\tilde{u}(x)+g_{\tilde{u}}(t) d_{X}(x, t)$, i.e. $g_{\tilde{u}}$ is constant and $\tilde{u}$ is linear (with respect to the metric $d_{X}$ ) on the edge $[x, y]$. It follows that $g_{\tilde{u}}$ is a minimal upper gradient of $\tilde{u}$ on the edge $[x, y]$. The contribution from this edge to $\int_{X} g_{\tilde{u}}^{p} d \mu$ is

$$
\int_{[x, y]} g_{\tilde{u}}^{p} d \mu \simeq \int_{n}^{n+1}\left(\frac{\left|u_{n}(\zeta)-u_{n+1}(\zeta)\right|}{e^{-\varepsilon n}}\right)^{p} e^{-\beta \tau} d \tau \simeq e^{(\varepsilon p-\beta) n}\left|u_{n}(\zeta)-u_{n+1}(\zeta)\right|^{p} .
$$

Here, the choice of $\zeta \in \partial X$ is dictated by the child $y$ of $x$, but $\zeta$ can be replaced by any choice of $\xi \in B\left(\zeta, r_{n+1}\right)$. Integrating (6.8) over this smaller ball, we obtain

$$
\nu\left(B\left(\zeta, r_{n+1}\right)\right) \int_{[x, y]} g_{\tilde{u}}^{p} d \mu \simeq e^{(\varepsilon p-\beta) n} \int_{B\left(\zeta, r_{n+1}\right)}\left|u_{n}(\xi)-u_{n+1}(\xi)\right|^{p} d \nu(\xi) .
$$

Summing over all the edges in $X$ connecting vertices at level $n$ to vertices at level $n+1$ shows that the contribution to $\int_{X} g_{\tilde{u}}^{p} d \mu$ from all such edges is comparable to

$$
e^{(\varepsilon p-\beta) n} \int_{\partial X} \frac{\left|u_{n}(\zeta)-u_{n+1}(\zeta)\right|^{p}}{\nu\left(B\left(\zeta, r_{n+1}\right)\right)} d \nu(\zeta)
$$

Summing over all $n \in \mathbb{N}$ and writing $\left|u_{n}(\zeta)-u_{n+1}(\zeta)\right| \leq\left|v_{n}(\zeta)\right|+\left|v_{n+1}(\zeta)\right|$, where 
$v_{n}=u_{n}-u$, we obtain from (5.13) that

$$
\begin{aligned}
\int_{X} g_{\tilde{u}}^{p} d \mu & \lesssim \sum_{n=0}^{\infty} e^{(\varepsilon p-\beta) n} \int_{\partial X} \frac{\left(\left|v_{n}(\zeta)\right|+\left|v_{n+1}(\zeta)\right|\right)^{p}}{\nu\left(B\left(\zeta, r_{n+1}\right)\right)} d \nu(\zeta) \\
& \lesssim \sum_{n=0}^{\infty} \frac{e^{(\varepsilon p-\beta) n}}{r_{n}^{Q}} \int_{\partial X} f_{B\left(\zeta, r_{n}\right)}|u(\chi)-u(\zeta)|^{p} d \nu(\chi) d \nu(\zeta) \\
& \simeq \sum_{n=0}^{\infty} \frac{e^{(\varepsilon p-\beta) n} r_{n}^{\theta p}}{r_{n}^{Q}}\left(\frac{E_{p}\left(u, r_{n}\right)}{r_{n}^{\theta}}\right)^{p} .
\end{aligned}
$$

Since $r_{n} \simeq e^{-n \varepsilon}$, Lemma 5.4 shows that the sum converges provided that

$$
e^{(\varepsilon p-\beta) n} r_{n}^{\theta p-Q} \simeq e^{(\varepsilon p-\beta-\varepsilon(\theta p-Q)) n} \leq C
$$

for all $n=0,1, \ldots$, i.e. if $\varepsilon p-\beta-\varepsilon(\theta p-Q) \leq 0$. This is equivalent to $\theta \geq$ $1+Q / p-\beta / \varepsilon p$, which is (6.5). Thus $\left\|g_{\tilde{u}}\right\|_{L^{p}(X)} \lesssim\|u\|_{B_{p, p}^{\theta}(\partial X)}$ for such $\theta$.

The finiteness of $\|\tilde{u}\|_{L^{p}(X)}$ now follows from the $(p, p)$-Poincaré inequality (Corollary 4.3$)$, since $\tilde{u} \in L^{1}(X)$ by the construction of $\tilde{u}$.

Combining Propositions 6.1 and 6.4 we obtain the following theorem identifying the trace space.

Theorem 6.5. Let $X$ be a regular $K$-ary tree with the metric $d_{X}$ defined by the exponential weight as in (2.1) with $\varepsilon>0$ and the measure $\mu$ defined by the exponential weight with $\beta>\log K$, and let $p \geq 1$. Then the trace space of $N^{1, p}(X)$ is the Besov space $B_{p, p}^{\theta}(\partial X)$, where $\theta=1-(\beta-\log K) / p \varepsilon$, with equivalent norms.

The following embedding result follows from Proposition 6.4 by means of an embedding theorem for Newtonian spaces.

In the Euclidean setting, it is well known that the Besov spaces $B_{p, p}^{\theta}\left(\mathbb{R}^{n}\right)$ and $B_{p, p}^{\theta}(\Omega)$ for smooth Euclidean subdomains $\Omega$ embed continuously into $C^{\alpha}$ provided that $\theta=\alpha+n / p$, see e.g. Triebel [47, Section 2.7.1]. Our result extends this to regular Cantor type sets.

Proposition 6.6. Let $X$ be a regular $K$-ary tree with the metric $d_{X}$ defined as in (2.1) by the exponential weight with $\varepsilon>0$. Let $Q=(\log K) / \varepsilon$ be the Hausdorff dimension of $\partial X$ and $p>1$. Then $B_{p, p}^{\theta}(\partial X) \subset C^{\alpha}(\partial X)$, in the sense that every $u \in B_{p, p}^{\theta}(\partial X)$ has an $\alpha$-Hölder continuous representative, provided that one of the following conditions holds:

(a) $Q<1, \theta \geq \frac{Q-1}{p}+1$ and $\alpha=1-\frac{1}{p}>0$;

(b) $\frac{Q}{p}<\theta \leq \frac{Q-1}{p}+1, \theta<1$ and $\alpha=\theta-\frac{Q}{p}$;

(c) $Q \geq 1, \theta \geq 1$ and $0<\alpha<1-\frac{Q}{p}$.

Proof. (a) and (b) Equip $X$ with the measure $d \mu=e^{-\beta|x|} d|x|$ as in (3.1), where $\beta=\varepsilon$ in (a) and $\beta=Q \varepsilon+p \varepsilon(1-\theta) \geq \varepsilon$ in (b). Note that in both cases, $\theta \geq 1-$ $(\beta-\log K) / p \varepsilon$ and $\beta>\log K$. Proposition 6.4 then shows that every $u \in B_{p, p}^{\theta}(\partial X)$ can be extended to $U \in N^{1, p}(X)$ so that for $\nu$-a.e. $\zeta \in \partial X, U(x) \rightarrow u(\zeta)$ as $x \rightarrow \zeta$ along a geodesic. By Corollary 3.8, $\mu$ satisfies (3.9) with $s=\beta / \varepsilon \geq 1$. Note also that $p>s$, both in (a) and (b). It follows that $N^{1, p}(X)$ embeds continuously into $C^{1-s / p}(X)$ with respect to the metric $d_{X}$, by e.g. Theorem 5.1 in HajłaszKoskela [22]. Hence, the function $u^{*}(\zeta):=\lim _{x \rightarrow \zeta} U(x)$ is well defined and $(1-s / p)$ Hölder continuous on $\partial X$. Since $1-s / p=\alpha$ and $u^{*}=u \nu$-a.e., the result follows in this case. 
(c) Let $\tau=\alpha+Q / p$ and note that $Q / p<\tau \leq Q / p+1-1 / p, \tau<1$ and $\alpha=\tau-Q / p$, i.e. (b) holds with $\theta$ replaced by $\tau$. The already proved part (b) together with (5.6) then gives the result.

\section{Quasisymmetric mappings}

In this section we begin a discussion of quasisymmetric mappings between boundaries of two trees. We will assume that the boundaries are uniformly perfect and equipped with Ahlfors regular measures. We shall show that if $f: \partial X \rightarrow \partial Y$ is a quasisymmetric mapping satisfying a certain dimension condition, then for every $p \geq 1$ and $\theta>0$ there is a bounded induced map $f_{\#}: B_{p, p}^{\theta}(\partial Y) \rightarrow B_{p, p}^{\tau}(\partial X)$ for some $\tau>0$. There are already indications in the current literature that certain Besov spaces may be invariant under quasisymmetries; see Bourdon-Pajot [11]. Hence this result is natural.

Primary examples for these investigations come from regular trees, but regularity is not strictly required. All we need is uniform perfectness (or rather the fact that the mapping under consideration is a power map) and Ahlfors regularity.

We therefore first formulate and prove our results for Besov spaces on general metric spaces and then specialize them to boundaries of trees. Recall that a metric space $Z$ is uniformly perfect if there is a constant $C>1$ such that whenever $z \in Z$ and $0<r<\operatorname{diam} Z$, the annulus $B(z, r) \backslash B(z, r / C)$ is nonempty. A measure $\nu$ on $Z$ is Ahlfors $Q$-regular if for every $z \in Z$ and $0<r<2 \operatorname{diam} Z$ we have $\nu(B(z, r)) \simeq r^{Q}$.

A homeomorphism $f: Z \rightarrow W$ between two metric spaces $\left(Z, d_{Z}\right)$ and $\left(W, d_{W}\right)$ is quasisymmetric if there is a homeomorphism $\eta:[0, \infty) \rightarrow[0, \infty)$ such that whenever $x, y, z \in Z$ and $x \neq z$, then

$$
\frac{d_{W}(f(x), f(y))}{d_{W}(f(x), f(z))} \leq \eta\left(\frac{d_{Z}(x, y)}{d_{Z}(x, z)}\right) .
$$

If $Z$ and $W$ are uniformly perfect then every quasisymmetric map between them has to be a power quasisymmetric map, see Heinonen [25, Theorem 11.3]. This means that $\eta$ can be chosen to be of the form

$$
\eta(t)= \begin{cases}A t^{\alpha}, & \text { if } t \leq 1 \\ A t^{1 / \alpha}, & \text { if } t \geq 1\end{cases}
$$

for some $0<\alpha \leq 1$ and $A>0$. Interchanging the role of $y$ and $z$ in the definition (7.1) of quasisymmetry gives a lower bound with the function $\varphi(t)=1 / \eta(1 / t)$. Thus we have the following pair of inequalities for all $x, y, z \in Z$ with $x \neq z$ :

$$
\varphi\left(\frac{d_{Z}(x, y)}{d_{Z}(x, z)}\right) \leq \frac{d_{W}(f(x), f(y))}{d_{W}(f(x), f(z))} \leq \eta\left(\frac{d_{Z}(x, y)}{d_{Z}(x, z)}\right) .
$$

It is easily verified that (7.2) yields

$$
\varphi(t)= \begin{cases}A^{-1} t^{1 / \alpha}, & \text { if } t \leq 1 \\ A^{-1} t^{\alpha}, & \text { if } t \geq 1\end{cases}
$$

where the constant $A$ is the one associated with $\eta$ in (7.2). Note that the constant 1 in (7.2) and (7.3) can easily be replaced by any other positive constant, by changing the constant $A$.

If $Z$ is bounded, then for $x \in Z$ we can choose $z \in Z$ such that $d_{Z}(x, z) \geq$ $\frac{1}{3} \operatorname{diam} Z$, and from such a choice of $z$ we get

$$
C_{1} d_{Z}(x, y)^{1 / \alpha} \leq d_{W}(f(x), f(y)) \leq C_{2} d_{Z}(x, y)^{\alpha},
$$


i.e. both $f$ and $f^{-1}$ are $\alpha$-Hölder.

We say that a bijective map $f: Z \rightarrow W$ is $\left(\alpha_{1}, \alpha_{2}\right)$-biHölder if there are constants $C_{1}, C_{2}>0$ such that for all $x, y \in Z$,

$$
C_{1} d_{Z}(x, y)^{\alpha_{1}} \leq d_{W}(f(x), f(y)) \leq C_{2} d_{Z}(x, y)^{\alpha_{2}} .
$$

If $\alpha_{1}=1 / \alpha$ and $\alpha_{2}=\alpha$, we say that $f$ is $\alpha$-biHölder.

Clearly, every $\alpha$-biHölder map is $(1 / \alpha, \alpha)$-biHölder, but there may be better constants $\alpha_{1}$ and $\alpha_{2}$ for which (7.4) holds. Conversely, if $Z$ is bounded then every $\left(\alpha_{1}, \alpha_{2}\right)$-biHölder map is $\alpha$-biHölder with $\alpha=\min \left\{1 / \alpha_{1}, \alpha_{2}\right\}$.

Lemma 7.1. Assume that $Z$ and $W$ are bounded metric spaces equipped with an Ahlfors $Q_{Z}$-regular measure $\nu_{Z}$ and an Ahlfors $Q_{W}$-regular measure $\nu_{W}$ respectively. Suppose that $f: Z \rightarrow W$ is an $\left(\alpha_{1}, \alpha_{2}\right)$-biHölder homeomorphism such that $Q_{Z} \geq$ $\alpha_{1} Q_{W}$. Then the map $f$ induces a bounded embedding $f_{\#}: L^{p}(W) \rightarrow L^{p}(Z)$ for $p \geq 1$, via composition.

The proof below does not need $f$ to be biHölder, it is sufficient to require that $f^{-1}$ is a $1 / \alpha_{1}$-Hölder continuous homeomorphism.

Proof. Since $Z$ and $W$ are separable, continuous functions are dense in $L^{p}(Z)$ and $L^{p}(W)$. Let $u \in L^{p}(W)$ be a continuous function. Then $\int_{W}|u|^{p} d \nu_{W}$ and $\int_{Z} \mid u \circ$ $\left.f\right|^{p} d \nu_{Z}$ can be computed using Riemann sums. Cover $Z$ by balls $B_{i}=B_{Z}\left(z_{i}, r\right)$ with common radius $r<1$ so that the balls $B_{Z}\left(z_{i}, \frac{1}{2} r\right)$ are pairwise disjoint and

$$
\int_{Z}|u \circ f|^{p} d \nu_{Z} \simeq \sum_{i}\left|u \circ f\left(z_{i}\right)\right|^{p} \nu_{Z}\left(B_{i}\right)
$$

for some choice $z_{i} \in B_{i}$. The Ahlfors regularity condition then implies that the balls $\left\{B_{i}\right\}_{i}$ have bounded overlap with a bound independent of $r$. Since $f$ is $\left(\alpha_{1}, \alpha_{2}\right)$ biHölder continuous, we know that $B_{W}\left(f\left(z_{i}\right), C r^{\alpha_{1}}\right) \subset f\left(B_{i}\right)$ and hence

$$
\nu_{Z}\left(B_{i}\right) \simeq r^{Q_{Z}} \leq r^{\alpha_{1} Q_{W}} \lesssim \nu_{W}\left(f\left(B_{i}\right)\right) .
$$

Thus, we have

$$
\int_{Z}|u \circ f|^{p} d \nu_{Z} \lesssim \sum_{i}\left|u \circ f\left(z_{i}\right)\right|^{p} \nu_{W}\left(f\left(B_{i}\right)\right) .
$$

As $f$ is a homeomorphism and $\left\{B_{i}\right\}_{i}$ have bounded overlap, so do $\left\{f\left(B_{i}\right)\right\}_{i}$, and letting $r \rightarrow 0$ we obtain

$$
\int_{Z}|u \circ f|^{p} d \nu_{Z} \lesssim \limsup _{r \rightarrow 0} \sum_{i}\left|u \circ f\left(z_{i}\right)\right|^{p} \nu_{W}\left(f\left(B_{i}\right)\right) \lesssim \int_{W}|u|^{p} d \nu_{W} .
$$

By the density of continuous functions in the corresponding $L^{p}$-spaces, we see that $f_{\#}$ boundedly embeds $L^{p}(Z)$ into $L^{p}(W)$.

The following proposition is a simple consequence of Lemma 7.1 and provides us with embeddings between Besov spaces.

Proposition 7.2. Assume that $Z$ and $W$ are bounded metric spaces equipped with an Ahlfors $Q_{Z}$-regular measure $\nu_{Z}$ and an Ahlfors $Q_{W}$-regular measure $\nu_{W}$ respectively. Let $f: Z \rightarrow W$ be an $\left(\alpha_{1}, \alpha_{2}\right)$-biHölder homeomorphism such that

$$
Q_{Z} \geq \alpha_{1} Q_{W},
$$

and $\theta, \tau>0$ and $p \geq 1$ be such that

$$
\tau \leq \alpha_{2} \theta+\frac{\alpha_{2} Q_{W}-Q_{Z}}{p}
$$

Then $f$ induces a canonical bounded embedding $f_{\#}: B_{p, p}^{\theta}(W) \rightarrow B_{p, p}^{\tau}(Z)$ via composition. 
Proposition 7.2 can immediately be applied to the tree boundaries $Z=\partial X$ and $W=\partial Y$, considered in the previous sections, provided that each vertex in $X$ and $Y$ has at least two children (so that the boundaries are uniformly perfect) and the boundaries $\partial X$ and $\partial Y$ are Ahlfors regular. The Ahlfors regularity is guaranteed e.g. if $X$ and $Y$ are regular trees, but can hold also in less regular situations. The metrics on $\partial X$ and $\partial Y$ are the visual metrics given by (5.1). Such metrics can be defined also for nonregular trees.

An $\left(\alpha_{1}, \alpha_{2}\right)$-biHölder homeomorphism $f: \partial X \rightarrow \partial Y$ thus induces a canonical bounded embedding $f_{\#}: B_{p, p}^{\theta}(\partial Y) \rightarrow B_{p, p}^{\tau}(\partial X)$, provided that $Q_{X} \geq \alpha_{1} Q_{Y}$ and

$$
\tau \leq \alpha_{2} \theta+\frac{\alpha_{2} Q_{Y}-Q_{X}}{p}
$$

In particular, this applies to quasisymmetric mappings between the boundaries with $\alpha_{1}=\alpha^{-1}$ and $\alpha_{2}=\alpha$ for some $0<\alpha \leq 1$.

Proof of Proposition 7.2. Suppose that $u \in B_{p, p}^{\theta}(W)$, and let $v=u \circ f$. Also let $\theta_{0}=\theta+Q_{W} / p$ and $\tau_{0}=\tau+Q_{Z} / p$. For $x, z \in Z$, we have by the Hölder continuity of $f$ and the fact that $\tau_{0} \leq \alpha_{2} \theta_{0}$,

$$
\begin{aligned}
\frac{|v(z)-v(x)|}{d_{Z}(z, x)^{\tau_{0}}} & =\frac{|v(z)-v(x)|}{d_{W}(f(z), f(x))^{\theta_{0}}} \frac{d_{W}(f(z), f(x))^{\theta_{0}}}{d_{Z}(z, x)^{\tau_{0}}} \\
& \lesssim \frac{|v(z)-v(x)|}{d_{W}(f(z), f(x))^{\theta_{0}}} d_{Z}(z, x)^{\alpha_{2} \theta_{0}-\tau_{0}} \lesssim \frac{|u \circ f(z)-u \circ f(x)|}{d_{W}(f(z), f(x))^{\theta_{0}}}
\end{aligned}
$$

By the second part of Lemma 5.4, we see that

$$
\|v\|_{B_{p, p}^{\tau}(Z)}^{p} \simeq \int_{Z} \int_{Z}\left(\frac{|v(z)-v(x)|}{d_{Z}(z, x)^{\tau_{0}}}\right)^{p} d \nu_{Z}(z) d \nu_{Z}(x) .
$$

Inequality (7.7) and Lemma 7.1 then yield,

$$
\begin{aligned}
\|v\|_{B_{p, p}^{\tau}(Z)}^{p} & \lesssim \int_{Z} \int_{Z}\left(\frac{|u \circ f(z)-u \circ f(x)|}{d_{W}(f(z), f(x))^{\theta_{0}}}\right)^{p} d \nu_{Z}(z) d \nu_{Z}(x) \\
& \lesssim \int_{W} \int_{W}\left(\frac{|u(y)-u(w)|}{d_{W}(y, w)^{\theta_{0}}}\right)^{p} d \nu_{W}(y) d \nu_{W}(w) \simeq\|u\|_{B_{p, p}^{\theta}(W)}^{p}
\end{aligned}
$$

Thus we have a bounded embedding $f_{\#}: B_{p, p}^{\theta}(W) \rightarrow B_{p, p}^{\tau}(Z)$, with control over the Besov seminorm. Control of the $L^{p}$ norm is given by Lemma 7.1.

Remark 7.3. Assume that the metric space $Z$ is equipped with another "snowflaked" metric $d_{Z}^{\prime}$ satisfying

$$
d_{Z}^{\prime}(z, x)=d_{Z}(z, x)^{\sigma}
$$

for some $\sigma>0$ and all $z, x \in Z$. Then the ball $B(z, r)$ with respect to $d_{Z}$ equals the ball $B^{\prime}\left(z, r^{\prime}\right)$ with respect to $d_{Z}^{\prime}$, where $r^{\prime}=r^{\sigma}$, and $\nu_{Z}(B(\zeta, r))=\nu_{Z}\left(B^{\prime}\left(z, r^{\prime}\right)\right)$. This means that the Ahlfors regularity exponent changes accordingly, i.e. $Q_{Z}^{\prime}=$ $Q_{Z} / \sigma$. Also, it is easily verified that $B_{p, p}^{\tau}(Z)$ with respect to $d_{Z}$ equals $B_{p, p}^{\tau^{\prime}}(Z)$ with respect to $d_{Z}^{\prime}$, where $\tau^{\prime}=\tau / \sigma$.

A similar observation holds for $W$ with $d_{W}^{\prime}(y, w)=d_{W}(y, w)^{\kappa}$ and $\theta^{\prime}=\theta / \kappa$. We also see that an $\left(\alpha_{1}, \alpha_{2}\right)$-biHölder map $f:\left(Z, d_{Z}\right) \rightarrow\left(W, d_{W}\right)$ is $\left(\alpha_{1}^{\prime}, \alpha_{2}^{\prime}\right)$-biHölder when regarded as a map from $\left(Z, d_{Z}^{\prime}\right)$ to $\left(W, d_{W}^{\prime}\right)$, with $\alpha_{j}^{\prime}=\alpha_{j} \kappa / \sigma, j=1,2$.

Note that the conditions (7.5) and (7.6) remain invariant under such changes, and thus Lemma 7.1 and Proposition 7.2 are invariant under "snowflaking" of the metrics. 
For $0<\sigma \leq 1$ the snowflaking in (7.8) always gives a new metric, but this is not true in general for $\sigma>1$. However, for our primary examples, boundaries of trees, snowflaking always produces a new metric also for $\sigma>1$. Changing the weight exponents $\varepsilon_{X}$ and $\varepsilon_{Y}$ which determine the metrics on $\partial X$ and $\partial Y$ to $\varepsilon_{X}^{\prime}$ and $\varepsilon_{Y}^{\prime}$ respectively, gives new "snowflaked" visual metrics $d_{X}^{\prime} \simeq d_{X}^{\sigma}$ and $d_{Y}^{\prime} \simeq d_{Y}^{\kappa}$ with $\sigma=\varepsilon_{X}^{\prime} / \varepsilon_{X}$ and $\kappa=\varepsilon_{Y}^{\prime} / \varepsilon_{Y}$. Thus the identity is a quasisymmetric self-mapping of $\partial X$ and of $\partial Y$ and it follows that if $f: \partial X \rightarrow \partial Y$ is a quasisymmetric map for some $\varepsilon_{X}, \varepsilon_{Y}>0$ then it is quasisymmetric with respect to all metrics on $\partial X$ and $\partial Y$ given by (5.1).

The conditions $Q_{X} \geq \alpha_{1} Q_{Y}$ and $\tau \leq \alpha_{2} \theta+p^{-1}\left(\alpha_{2} Q_{Y}-Q_{X}\right)$ place restrictions on the type of biHölder maps for which Proposition 7.2 holds and on the Hausdorff dimensions of $\partial X$ and $\partial Y$. The result applies to general metric spaces but does not take the geometry of the spaces into consideration. Our primary examples $\partial X$ and $\partial Y$ are Cantor type sets with more structure than general metric spaces, and Besov spaces on such sets can be regarded as traces of Newtonian spaces. Thus the conclusions of Proposition 7.2 can be improved in this case by exploiting embeddings between Newtonian spaces on trees. To do so, we need to extend quasisymmetric mappings between boundaries of trees to nice mappings between the trees. This will be the focus of the next section. The corresponding result for Besov spaces appears in Theorem 8.3 below.

\section{Extending quasisymmetries from the boundary to the tree, and embeddings of Besov spaces}

In this section, we assume that both $X$ and $Y$ are rooted trees such that each vertex has at least two children. In particular, $\partial X$ and $\partial Y$ are uniformly prefect, but no regularity is assumed (except for Theorem 8.3)

Proposition 7.2 and the comments after it show that quasisymmetric mappings between boundaries of two trees preserve Besov spaces. At the same time, we saw in Theorem 6.5 that Besov functions on the boundary of a regular tree are traces of (and extend to) Newtonian functions on the tree. It is therefore natural to ask whether every quasisymmetric map can be extended to the tree as some mapping preserving Newtonian spaces in a reasonable way. The aim of this section is to study this question. The natural property of such extended functions is the rough quasiisometry, see Definition 8.1.

We shall show that every quasisymmetry between the boundaries of two trees extends to a rough quasiisometry between the trees. At the end of this section, in Theorem 8.3, we will show that if the trees involved are regular, then the quasisymmetry between their boundaries induces a canonical bounded linear map between certain Besov spaces, under less restrictive assumptions than those in Proposition 7.2.

Let $X$ and $Y$ be two trees such that each vertex has at least two children. Let $d_{X}$ and $d_{Y}$ be the metrics on $X$ and $Y$ given as in (2.1) by the exponential weights with $\varepsilon_{X}$ and $\varepsilon_{Y}$, respectively. Consider the Cantor type boundaries $\partial X$ and $\partial Y$ with respect to these metrics and extend the metrics to the boundaries as in (5.1). Also let $|\cdot-\cdot|$ denote the metric on the vertices of a tree given by the number of edges in the geodesic from one vertex to another. Recall that $|x|=\left|x-0_{X}\right|$ and $|y|=\left|y-0_{Y}\right|$ for $x \in X$ and $y \in Y$.

Assume that $f: \partial X \rightarrow \partial Y$ is an $\eta$-quasisymmetry, as in (7.1). Since each vertex in $X$ and $Y$ has more than one child, the boundaries $\partial X$ and $\partial Y$ are uniformly 
perfect and $\eta$ can be chosen to be of the form

$$
\eta(t)= \begin{cases}A t^{\alpha}, & \text { if } t \leq 1 \\ A t^{1 / \alpha}, & \text { if } t \geq 1\end{cases}
$$

for some $\alpha \leq 1$ and $A>0$, by Theorem 11.3 in Heinonen [25].

We now use $f$ to construct a map $F: X \rightarrow Y$ as follows: For $x \in X$ we let $F(x)$ be the largest vertex in $Y$ (with respect to $\leq$ ) with the property that

$$
f(\xi)>F(x) \text { for all } \xi>x .
$$

In other words, we consider all the descendants $\xi \in \partial X$ of $x$, and choose as $F(x)$ the largest common ancestor of all $f(\xi)$ for these $\xi$. Thus $F: X \rightarrow Y$ is defined. We shall use (7.1) to discover properties of this map, namely for arbitrary $x_{1}, x_{2} \in X$ we want bounds for $\left|F\left(x_{1}\right)-F\left(x_{2}\right)\right|$ in terms of $\left|x_{1}-x_{2}\right|$.

Definition 8.1. A (not necessarily continuous) mapping $F: X \rightarrow Y$ is an $(L, \Lambda)$ rough quasiisometry if whenever $x, y \in X$ we have

$$
\frac{1}{L}|x-y|-\Lambda \leq|F(x)-F(y)| \leq L|x-y|+\Lambda,
$$

and for each $y \in Y$ there is a point $x \in X$ such that $|F(x)-y| \leq L+\Lambda$.

Such maps can be regarded as mappings between the vertices of the corresponding trees, or as mappings between the edge-connected trees (by mapping the edge between the two vertices to the geodesic connecting the images of the two vertices). This dichotomy of rough quasiisometries will be exploited in this and subsequent sections of this paper. Much of the current literature on rough quasiisometries call such maps quasiisometries. However, we will follow the terminology of BonkHeinonen-Koskela [8] to avoid confusion with biLipschitz maps.

Theorem 8.2. Let $X$ and $Y$ be two rooted trees, such that each vertex has at least two children. Let $\varepsilon_{X}$ and $\varepsilon_{Y}$ give the weighted metrics on $X$ and $Y$ respectively as in (5.1). Suppose that $f: \partial X \rightarrow \partial Y$ is an $\eta$-quasisymmetric map, where

$$
\eta(t)=\left\{\begin{array}{ll}
A t^{\alpha_{1}}, & \text { if } t \leq 1, \\
A t^{\alpha_{2}}, & \text { if } t \geq 1,
\end{array} \quad \text { and } \quad \alpha_{1}, \alpha_{2}, A>0 .\right.
$$

Then there is an $(L, \Lambda)$-rough quasiisometry $F: X \rightarrow Y$ which extends continuously along geodesics in $X$ to $f$, and such that for all $x_{1}, x_{2} \in X$,

$$
L_{1}\left|x_{1}-x_{2}\right|-\Lambda \leq\left|F\left(x_{1}\right)-F\left(x_{2}\right)\right| \leq L_{2}\left|x_{1}-x_{2}\right|+\Lambda,
$$

where

$$
L_{1}=\frac{\alpha_{1} \varepsilon_{X}}{\varepsilon_{Y}}, \quad L_{2}=\frac{\alpha_{2} \varepsilon_{X}}{\varepsilon_{Y}}, \quad L=\max \left\{\frac{1}{L_{1}}, L_{2}\right\} \quad \text { and } \quad \Lambda=\frac{2 \log A}{\varepsilon_{Y}} .
$$

The formulas for $L_{1}$ and $L_{2}$ (and thus also for $L$ ) are sharp as we will see later in Remark 9.10.

Proof. Let $x_{1}, x_{2} \in X$. We consider two cases.

Case 1. $x_{1}$ and $x_{2}$ are comparable with respect to the partial order $\leq$. In this case we can without loss of generality assume that $x_{2}>x_{1}$, i.e.

$$
\left|x_{1}-x_{2}\right|=\left|x_{2}\right|-\left|x_{1}\right| \text {. }
$$


It is then clear by the definition of $F$ that $F\left(x_{1}\right) \leq F\left(x_{2}\right)$, i.e.

$$
\left|F\left(x_{1}\right)-F\left(x_{2}\right)\right|=\left|F\left(x_{2}\right)\right|-\left|F\left(x_{1}\right)\right| .
$$

Using (5.1) and the fact that $F\left(x_{1}\right)$ has at least two children, we can find $\xi^{*}, \xi_{1} \in \partial X$ so that $\xi_{1}>x_{1}, \xi^{*}>x_{2}$ and

$$
d_{Y}\left(f\left(\xi^{*}\right), f\left(\xi_{1}\right)\right)=\frac{2}{\varepsilon_{Y}} e^{-\varepsilon_{Y}\left|F\left(x_{1}\right)\right|} .
$$

Since $x_{2}$ has at least two children, we can also find $\partial X \ni \xi_{2}>x_{2}$ such that $d_{X}\left(\xi^{*}, \xi_{2}\right)=2 e^{-\varepsilon_{X}\left|x_{2}\right|} / \varepsilon_{X}$. Then

$$
\frac{d_{X}\left(\xi^{*}, \xi_{1}\right)}{d_{X}\left(\xi^{*}, \xi_{2}\right)} \leq \frac{2 e^{-\varepsilon_{X}\left|x_{1}\right|} / \varepsilon_{X}}{2 e^{-\varepsilon_{X}\left|x_{2}\right|} / \varepsilon_{X}}=e^{\varepsilon_{X}\left|x_{1}-x_{2}\right|}>1
$$

and

$$
\frac{d_{Y}\left(f\left(\xi^{*}\right), f\left(\xi_{1}\right)\right)}{d_{Y}\left(f\left(\xi^{*}\right), f\left(\xi_{2}\right)\right)} \geq \frac{2 e^{-\varepsilon_{Y}\left|F\left(x_{1}\right)\right|} / \varepsilon_{Y}}{2 e^{-\varepsilon_{Y}\left|F\left(x_{2}\right)\right|} / \varepsilon_{Y}}=e^{\varepsilon_{Y}\left|F\left(x_{1}\right)-F\left(x_{2}\right)\right|} .
$$

The $\eta$-quasisymmetry condition now yields

$$
e^{\varepsilon_{Y}\left|F\left(x_{1}\right)-F\left(x_{2}\right)\right|} \leq \eta\left(\frac{d_{X}\left(\xi^{*}, \xi_{1}\right)}{d_{X}\left(\xi^{*}, \xi_{2}\right)}\right) \leq \eta\left(e^{\varepsilon_{X}\left|x_{1}-x_{2}\right|}\right) \leq A e^{\alpha_{2} \varepsilon_{X}\left|x_{1}-x_{2}\right|} .
$$

From this we conclude that

$$
\left|F\left(x_{1}\right)-F\left(x_{2}\right)\right| \leq \frac{\alpha_{2} \varepsilon_{X}}{\varepsilon_{Y}}\left|x_{1}-x_{2}\right|+\frac{\log A}{\varepsilon_{Y}} .
$$

For the converse inequality, first find $\xi, \xi_{2} \in \partial X$ so that $\xi, \xi_{2}>x_{2}$ and

$$
d_{Y}\left(f(\xi), f\left(\xi_{2}\right)\right)=\frac{2}{\varepsilon_{Y}} e^{-\varepsilon_{Y}\left|F\left(x_{2}\right)\right|},
$$

and then $\partial X \ni \xi_{1}>x_{1}$ so that $d_{X}\left(\xi, \xi_{1}\right)=2 e^{-\varepsilon_{X}\left|x_{1}\right|} / \varepsilon_{X}$. This is possible since both $F\left(x_{2}\right)$ and $x_{1}$ have at least two children. Then

$$
\frac{d_{X}\left(\xi, \xi_{2}\right)}{d_{X}\left(\xi, \xi_{1}\right)} \leq \frac{2 e^{-\varepsilon_{X}\left|x_{2}\right|} / \varepsilon_{X}}{2 e^{-\varepsilon_{X}\left|x_{1}\right|} / \varepsilon_{X}}=e^{-\varepsilon_{X}\left|x_{1}-x_{2}\right|}<1
$$

and

$$
\frac{d_{Y}\left(f(\xi), f\left(\xi_{2}\right)\right)}{d_{Y}\left(f(\xi), f\left(\xi_{1}\right)\right)} \geq \frac{2 e^{-\varepsilon_{Y}\left|F\left(x_{2}\right)\right|} / \varepsilon_{Y}}{2 e^{-\varepsilon_{Y}\left|F\left(x_{1}\right)\right|} / \varepsilon_{Y}}=e^{-\varepsilon_{Y}\left|F\left(x_{1}\right)-F\left(x_{2}\right)\right|} .
$$

The $\eta$-quasisymmetry condition now yields

$$
e^{-\varepsilon_{Y}\left|F\left(x_{1}\right)-F\left(x_{2}\right)\right|} \leq A e^{-\alpha_{1} \varepsilon_{X}\left|x_{1}-x_{2}\right|},
$$

and we conclude that

$$
\left|F\left(x_{1}\right)-F\left(x_{2}\right)\right| \geq \frac{\alpha_{1} \varepsilon_{X}}{\varepsilon_{Y}}\left|x_{1}-x_{2}\right|-\frac{\log A}{\varepsilon_{Y}} .
$$

Case 2. $x_{1}$ and $x_{2}$ are not comparable with respect to the partial order $\leq$. Let $\hat{x}$ be the largest common ancestor of $x_{1}$ and $x_{2}$ in $X$, and $\hat{y}$ be the largest common ancestor of $F\left(x_{1}\right)$ and $F\left(x_{2}\right)$ in $Y$. (Note that it is possible to have $\hat{y}$ be one of $F\left(x_{2}\right), F\left(x_{1}\right)$. Indeed, if $F\left(x_{1}\right)$ lies in the geodesic connecting $F(\hat{x})$ to $F\left(x_{2}\right)$, then necessarily $\hat{y}=F\left(x_{1}\right)$.) 
We obtain from (8.4) that

$$
\begin{aligned}
\left|F\left(x_{1}\right)-F\left(x_{2}\right)\right| & \leq\left|F\left(x_{1}\right)-F(\hat{x})\right|+\left|F(\hat{x})-F\left(x_{2}\right)\right| \\
& \leq \frac{\alpha_{2} \varepsilon_{X}}{\varepsilon_{Y}}\left(\left|x_{1}-\hat{x}\right|+\left|\hat{x}-x_{2}\right|\right)+\frac{2 \log A}{\varepsilon_{Y}} .
\end{aligned}
$$

Since $x_{1}$ and $x_{2}$ are not comparable, we have

$$
\left|x_{1}-x_{2}\right|=\left|x_{1}-\hat{x}\right|+\left|\hat{x}-x_{2}\right|
$$

and hence

$$
\left|F\left(x_{1}\right)-F\left(x_{2}\right)\right| \leq \frac{\alpha_{2} \varepsilon_{X}}{\varepsilon_{Y}}\left|x_{1}-x_{2}\right|+\frac{2 \log A}{\varepsilon_{Y}} .
$$

For the converse inequality, we let $\xi_{1}, \xi_{2} \in \partial X$ be such that $\xi_{1}>x_{1}$ and $\xi_{2}>x_{2}$. Since $\hat{y}$ is the largest common ancestor of $F\left(x_{1}\right)$ and $F\left(x_{2}\right)$, which in turn are ancestors of $f\left(\xi_{1}\right)$ and $f\left(\xi_{2}\right)$, we see that

$$
d_{Y}\left(f\left(\xi_{1}\right), f\left(\xi_{2}\right)\right) \leq 2 e^{-\varepsilon_{Y}|\hat{y}|} / \varepsilon_{Y} .
$$

By the definition of $F\left(x_{1}\right)$, we can choose a descendant $\xi_{1}^{\prime} \in \partial X$ of $x_{1}$ so that $d_{Y}\left(f\left(\xi_{1}\right), f\left(\xi_{1}^{\prime}\right)\right)=2 e^{-\varepsilon_{Y}\left|F\left(x_{1}\right)\right|} / \varepsilon_{Y}$ and hence

$$
\frac{d_{Y}\left(f\left(\xi_{1}\right), f\left(\xi_{1}^{\prime}\right)\right)}{d_{Y}\left(f\left(\xi_{1}\right), f\left(\xi_{2}\right)\right)} \geq \frac{2 e^{-\varepsilon_{Y}\left|F\left(x_{1}\right)\right|} / \varepsilon_{Y}}{2 e^{-\varepsilon_{Y}|\hat{y}|} / \varepsilon_{Y}}=e^{\varepsilon_{Y}\left(|\hat{y}|-\left|F\left(x_{1}\right)\right|\right)} .
$$

As $x_{1}<\xi_{1} \in \partial X$ and $x_{2}<\xi_{2} \in \partial X$, we see that the geodesic from $\xi_{1}$ to $\xi_{2}$ must pass through $\hat{x}$. Hence by (5.1),

$$
d_{X}\left(\xi_{1}, \xi_{2}\right)=2 e^{-\varepsilon_{Y}|\hat{x}|} / \varepsilon_{X} .
$$

Since moreover $d_{X}\left(\xi_{1}, \xi_{1}^{\prime}\right) \leq 2 e^{-\varepsilon_{X}\left|x_{1}\right|} / \varepsilon_{X}$, and $|\hat{x}|<\left|x_{1}\right|$ we obtain that

$$
\frac{d_{X}\left(\xi_{1}, \xi_{1}^{\prime}\right)}{d_{X}\left(\xi_{1}, \xi_{2}\right)} \leq \frac{2 e^{-\varepsilon_{X}\left|x_{1}\right|} / \varepsilon_{X}}{2 e^{-\varepsilon_{X}|\hat{x}|} / \varepsilon_{X}}=e^{\varepsilon_{X}\left(|\hat{x}|-\left|x_{1}\right|\right)}<1 .
$$

The quasisymmetry condition (7.1) then implies that

$$
\frac{d_{Y}\left(f\left(\xi_{1}\right), f\left(\xi_{1}^{\prime}\right)\right)}{d_{Y}\left(f\left(\xi_{1}\right), f\left(\xi_{2}\right)\right)} \leq \eta\left(\frac{d_{X}\left(\xi_{1}, \xi_{1}^{\prime}\right)}{d_{X}\left(\xi_{1}, \xi_{2}\right)}\right) \leq \eta\left(e^{\varepsilon_{X}\left(|\hat{x}|-\left|x_{1}\right|\right)}\right) \leq A e^{\alpha_{1} \varepsilon_{X}\left(|\hat{x}|-\left|x_{1}\right|\right)} .
$$

Inserting this into (8.7) yields

$$
\varepsilon_{Y}\left(|\hat{y}|-\left|F\left(x_{1}\right)\right|\right) \leq \alpha_{1} \varepsilon_{X}\left(|\hat{x}|-\left|x_{1}\right|\right)+\log A .
$$

Since $F\left(x_{1}\right) \geq \hat{y}$ and $x_{1} \geq \hat{x}$, this gives

$$
\left|F\left(x_{1}\right)-\hat{y}\right| \geq \frac{\alpha_{1} \varepsilon_{X}}{\varepsilon_{Y}}\left|x_{1}-\hat{x}\right|-\frac{\log A}{\varepsilon_{Y}} .
$$

Similarly,

$$
\left|F\left(x_{2}\right)-\hat{y}\right| \geq \frac{\alpha_{1} \varepsilon_{X}}{\varepsilon_{Y}}\left|x_{2}-\hat{x}\right|-\frac{\log A}{\varepsilon_{Y}} .
$$

Summing up the last two estimates and using (8.6) gives

$$
\left|F\left(x_{1}\right)-F\left(x_{2}\right)\right|=\left|F\left(x_{1}\right)-\hat{y}\right|+\left|\hat{y}-F\left(x_{2}\right)\right| \geq \frac{\alpha_{1} \varepsilon_{X}}{\varepsilon_{Y}}\left|x_{1}-x_{2}\right|-\frac{2 \log A}{\varepsilon_{Y}}
$$

and proves the rough quasiisometry condition (8.3). 
To verify the "density property", let $y \in Y$, and $\chi \in \partial Y$ be a descendant of $y$, that is, $\chi>y$. Since $f$ is a quasisymmetry between $\partial X$ and $\partial Y$, it is surjective. It follows that there exists $\xi \in \partial X$ such that $f(\xi)=\chi$. Because $f$ is continuous, it also follows that for each $r>0$ there is a positive real number $\delta$ such that $f(B(\xi, \delta) \cap \partial X) \subset B(\chi, r) \cap \partial Y$. We therefore conclude that there is a vertex $y_{0} \in Y$ such that $\chi>y_{0} \geq y$ and a vertex $x \in X$ with $F(x)=y_{0}$. Let $Y_{0}$ be the collection of all such $y_{0}$.

Suppose that $y$ is not in the image of $X$ under $F$. Then $y \notin Y_{0}$. Let $z \in Y_{0}$ be minimal (with respect to $\leq$ ), i.e. such that whenever $y \leq w<z$ then $w \notin Y_{0}$. Let $X_{0}$ be the collection of all $x \in X$ for which $F(x)=z$. It is nonempty, since $z \in Y_{0}$. Let $v \in X_{0}$ be minimal, i.e. such that whenever $x<v$ then $x \notin X_{0}$.

Let $u \in X$ be the parent of $v$. Then $F(u)<F(v)=z$, by the monotonicity of $F$, and $|u-v|=1$. Since $z$ is minimal in $Y_{0}$, it follows that $F(u)<y<z=F(v)$ and hence

$$
|F(v)-y|<|F(v)-F(u)| \leq L|v-u|+\Lambda=L+\Lambda \text {. }
$$

Thus every $y \in Y$ is within a distance $L+\Lambda$ of the image of $X$ under $F$. This completes the proof of the theorem.

As promised at the end of Section 7, we now give a Besov space invariance result.

Theorem 8.3. Let $X$ and $Y$ be regular $K_{X}$ - and $K_{Y}$-ary trees equipped with metrics $d_{X}$ and $d_{Y}$ given by exponential weights with exponents $\varepsilon_{X}$ and $\varepsilon_{Y}$, as in (2.1).

Let $f: \partial X \rightarrow \partial Y$ be an $\eta$-quasisymmetric map with $\eta$ as in (8.2), and let $Q_{X}=\left(\log K_{X}\right) / \varepsilon_{X}$ and $Q_{Y}=\left(\log K_{Y}\right) / \varepsilon_{Y}$ be the Hausdorff dimensions of $\partial X$ and $\partial Y$ given by Lemma 5.2. Suppose that $p \geq 1$ and $\theta_{X}, \theta_{Y} \in(0,1)$ satisfy

$$
\theta_{X} \leq \begin{cases}\frac{Q_{X}}{p}+\alpha_{1}\left(\theta_{Y}-\frac{Q_{Y}}{p}\right), & \text { if } \theta_{Y} \geq \frac{Q_{Y}}{p} \\ \frac{Q_{X}}{p}+\alpha_{2}\left(\theta_{Y}-\frac{Q_{Y}}{p}\right), & \text { if } \theta_{Y} \leq \frac{Q_{Y}}{p}\end{cases}
$$

Then $f$ induces a canonical embedding $f_{\#}: B_{p, p}^{\theta_{Y}}(\partial Y) \rightarrow B_{p, p}^{\theta_{X}}(\partial X)$ such that when $u \in B_{p, p}^{\theta_{Y}}(\partial Y)$ is continuous, we have $f_{\#}(u)=u \circ f$.

This improves upon Proposition 7.2 for regular trees.

Remark 8.4. The inverse of an $\eta$-quasisymmetric mapping $f$, with $\eta$ as in (8.2), is $\tilde{\eta}$-quasisymmetric with

$$
\tilde{\eta}(t) \simeq \begin{cases}t^{1 / \alpha_{2}} & \text { for } t \leq 1 \\ t^{1 / \alpha_{1}} & \text { for } t \geq 1\end{cases}
$$

Thus $f^{-1}: \partial Y \rightarrow \partial X$ induces an embedding $f_{\#}^{-1}: B_{p, p}^{\theta_{X}}(\partial X) \rightarrow B_{p, p}^{\theta_{Y}^{\prime}}(\partial Y)$ whenever

$$
\theta_{Y}^{\prime} \leq \begin{cases}\frac{Q_{Y}}{p}+\frac{1}{\alpha_{2}}\left(\theta_{X}-\frac{Q_{X}}{p}\right), & \text { if } \theta_{X} \geq \frac{Q_{X}}{p} \\ \frac{Q_{Y}}{p}+\frac{1}{\alpha_{1}}\left(\theta_{X}-\frac{Q_{X}}{p}\right), & \text { if } \theta_{X} \leq \frac{Q_{X}}{p}\end{cases}
$$

When $\theta_{X}=Q_{X} / p$ and $\theta_{Y}=Q_{Y} / p$, this shows that (for all $\alpha_{1}$ and $\left.\alpha_{2}\right) f$ induces an equivalence of the Besov spaces $B_{p, p}^{\theta_{X}}(\partial X)$ and $B_{p, p}^{\theta_{Y}}(\partial Y)$. Thus, we recover Koskela-Yang-Zhou [38, Theorem 5.1] in our setting of boundaries of regular trees. 
We allow for other exponents as well, which gives more general results in our special setting. For example, we obtain the following embeddings with $\tau \geq 0$,

$$
\begin{aligned}
& B_{p, p}^{Q_{Y} / p+\tau / \alpha_{1}}(\partial Y) \longleftrightarrow B_{p, p}^{Q_{X} / p+\tau}(\partial X) \longleftrightarrow B_{p, p}^{Q_{Y} / p+\tau / \alpha_{2}}(\partial Y), \\
& B_{p, p}^{Q_{Y} / p-\tau / \alpha_{2}}(\partial Y) \longleftrightarrow B_{p, p}^{Q_{X} / p-\tau}(\partial X) \longleftrightarrow B_{p, p}^{Q_{Y} / p-\tau / \alpha_{1}}(\partial Y),
\end{aligned}
$$

which do not follow from Theorem 5.1 in [38] when $\tau>0$. In particular, if $\alpha_{1}=$ $\alpha_{2}=\alpha$ (i.e. $f$ is a "snowflaking" mapping), then $f$ induces an equivalence of the Besov spaces $B_{p, p}^{Q_{X} / p+\tau}(\partial X)$ and $B_{p, p}^{Q_{Y} / p+\tau / \alpha}(\partial Y)$ for all $\tau>-Q_{X} / p$.

To prove Theorem 8.3 we shall use the following lemma which, roughly speaking, gives a sufficient condition for an embedding between Newtonian spaces on trees.

Lemma 8.5. Let $X$ and $Y$ be rooted trees equipped with metrics $d_{X}, d_{Y}$ and measures $\mu_{X}, \mu_{Y}$ given by exponential weights with exponents $\varepsilon_{X}, \varepsilon_{Y}, \beta_{X}$ and $\beta_{Y}$, respectively, as in (2.1) and (3.1). Assume that each vertex in $X$ has at most $K_{X}$ number of children. Let $p \geq 1$ and assume that $F: X \rightarrow Y$ is an $(L, \Lambda)$-rough quasiisometry. We extend $F$ to the edges of $X$ so that the edge $[x, y] \subset X$ is mapped linearly (with respect to $d_{X}$ and $d_{Y}$ ) to the geodesic connecting $F(x)$ and $F(y)$ in $Y$. Also assume that there are a positive integer $n_{0}$ and a constant $C_{0}$ such that for all $x \in X$ with $|x| \geq n_{0}$,

$$
\left(p \varepsilon_{X}-\beta_{X}\right)|x|+\left(\beta_{Y}-p \varepsilon_{Y}\right)|F(x)| \leq C_{0} .
$$

Let $u: Y \rightarrow \mathbb{R}$ be linear (with respect to $d_{Y}$ ) on each edge, and let $g_{Y}$ be the minimal upper gradient of $u$ in $\left(Y, d_{Y}\right)$ given on each edge $[z, w] \subset Y$ by

$$
g_{Y}(\tau)=\frac{|u(z)-u(w)|}{d_{Y}(z, w)} \simeq e^{\varepsilon_{Y}|\tau|}|u(z)-u(w)| \quad \text { for } \tau \in[z, w] .
$$

Let $A=(L+\Lambda) e^{\varepsilon_{Y}(L+\Lambda)+\varepsilon_{X}}$. Then the function $g_{X}$ given on each edge $[x, y] \subset X$ by

$$
g_{X}(t)=\left\{\begin{array}{ll}
A e^{\varepsilon_{X}|t|-\varepsilon_{Y}|F(t)|} g_{Y}(F(t)), & \text { if } F(x) \neq F(y), \\
0, & \text { if } F(x)=F(y),
\end{array} \quad \text { for } t \in[x, y],\right.
$$

is an upper gradient of $v=u \circ F$ in $\left(X, d_{X}\right)$. Moreover,

$$
\left\|g_{X}\right\|_{L^{p}(X)} \lesssim\left\|g_{Y}\right\|_{L^{p}(Y)}
$$

Proof. That $g_{Y}$ is a minimal upper gradient of $u$ in $\left(Y, d_{Y}\right)$ is straightforward from its definition. Let $\gamma$ be a geodesic in $X$ connecting two points (not necessarily vertices) $a$ and $b$. By splitting $\gamma$ into parts if necessary, we can assume that $a$ and $b$ belong to the same edge. Let $\gamma^{\prime}$ be the geodesic in $Y$ connecting $F(a)$ to $F(b)$. Note that since $a$ and $b$ belong to the same edge, $\gamma^{\prime}=F \circ \gamma$. Then by the definition of upper gradients,

$$
|v(a)-v(b)|=|u(F(a))-u(F(b))| \leq \int_{\gamma^{\prime}} g_{Y} d_{Y} s
$$

where $d_{Y} s$ denotes the arc length measure on $\gamma^{\prime}$ with respect to the metric $d_{Y}$. The metric on $X$ is with respect to the density $e^{-\varepsilon_{X}|t|}$ and the metric on $Y$ is with respect to the density $e^{-\varepsilon_{Y}|t|}$ (see (2.1)). By the linearity (with respect to $d_{X}$ and $d_{Y}$ ) of $F$ on the edge $[x, y]$ containing $a$ and $b$, we have for all $t \in[a, b]$ that

$$
\frac{d_{Y} s}{d_{X} s}=\frac{d_{Y}(F(x), F(y))}{d_{X}(x, y)} \leq A \frac{e^{-\varepsilon_{Y}|F(t)|}}{e^{-\varepsilon_{X}|t|}}
$$


where $A$ is as in the statement of the lemma. Inserting this into (8.10) gives

$$
|v(a)-v(b)| \leq A \int_{\gamma} e^{\varepsilon_{X}|t|-\varepsilon_{Y}|F(t)|} g_{Y}(F(t)) d_{X} s(t)=\int_{\gamma} g_{X} d_{X} s,
$$

and shows that $g_{X}$ is an upper gradient of $v$ in $\left(X, d_{X}\right)$. (Note that if $F$ maps an edge $[x, y] \subset X$ to a single vertex $z \in Y$, then the above construction gives $g_{X} \equiv 0$ on $[x, y]$.) To estimate the $L^{p}$-norm of $g_{X}$, note first that

$$
\begin{aligned}
\int_{X} g_{X}^{p} d \mu_{X} & \simeq \sum_{\substack{x, y \in X \\
x \sim y}} \int_{[x, y]}\left(e^{\varepsilon_{X}|t|-\varepsilon_{Y}|F(t)|} g_{Y}(F(t))\right)^{p} d \mu_{X} \\
& \simeq \sum_{\substack{x, y \in X \\
x \sim y}} e^{\left(\varepsilon_{X}|x|-\varepsilon_{Y}|F(x)|\right) p-\beta_{X}|x|} \int_{[x, y]} g_{Y}(F(t))^{p} d t
\end{aligned}
$$

Since $|F(x)-F(y)| \leq L+\Lambda$ and $g_{X} \equiv 0$ on $[x, y]$ if $F(x)=F(y)$, we see that

$$
\int_{[x, y]} g_{Y}(F(t))^{p} d|t| \simeq \int_{[F(x), F(y)]} g_{Y}(\tau)^{p} d|\tau| \simeq e^{\beta_{Y}|F(x)|} \int_{[F(x), F(y)]} g_{Y}^{p} d \mu_{Y} .
$$

Inserting this into (8.11) and using (8.9) give

$$
\begin{aligned}
\int_{X} g_{X}^{p} d \mu_{X} & \simeq \sum_{\substack{x, y \in X \\
x \sim y}} e^{\left(p \varepsilon_{X}-\beta_{X}\right)|x|+\left(\beta_{Y}-p \varepsilon_{Y}\right)|F(x)|} \int_{[F(x), F(y)]} g_{Y}^{p} d \mu_{Y} \\
& \lesssim \sum_{\substack{x, y \in X \\
x \sim y}} \int_{[F(x), F(y)]} g_{Y}^{p} d \mu_{Y} .
\end{aligned}
$$

Now note that if $F(x)=F(y)$ for some $x, y \in X$, then $L^{-1}|x-y|-\Lambda \leq 0$, and so $|x-y| \leq L \Lambda$, showing that for every $z \in Y$ there are at most $\left(K_{X}+1\right)^{L \Lambda}$ elements in $F^{-1}(\{z\})$. Furthermore, since for every edge $[x, y] \subset X$ the geodesic connecting $F(x)$ to $F(y)$ contains at most $L+\Lambda$ edges, we can conclude that each edge in $Y$ belongs to at most $(L+\Lambda)\left(K_{X}+1\right)^{L \Lambda}$ geodesics connecting images of neighboring vertices of $X$. It follows that

$$
\sum_{\substack{x, y \in X \\ x \sim y}} \int_{[F(x), F(y)]} g_{Y}^{p} d \mu_{Y} \simeq \int_{Y} g_{Y}^{p} d \mu_{Y}
$$

which completes the proof.

Proof of Theorem 8.3. Note first that by Theorem 8.2, $f$ extends to a rough quasiisometry $F: X \rightarrow Y$ such that for all $x, y \in X$,

$$
L_{1}|x-y|-\Lambda \leq|F(x)-F(y)| \leq L_{2}|x-y|+\Lambda,
$$

where $L_{1}=\alpha_{1} \varepsilon_{X} / \varepsilon_{Y}$ and $L_{2}=\alpha_{2} \varepsilon_{X} / \varepsilon_{Y}$. Next, equip $X$ and $Y$ with measures $\mu_{X}$ and $\mu_{Y}$ given by exponential weights with exponents $\beta_{X}$ and $\beta_{Y}$, as in (3.1). Here

$$
\begin{aligned}
\beta_{X}=\log K_{X}+p \varepsilon_{X}\left(1-\theta_{X}\right) & =\left[Q_{X}+p\left(1-\theta_{X}\right)\right] \varepsilon_{X}, \\
\beta_{Y}=\log K_{Y}+p \varepsilon_{Y}\left(1-\theta_{Y}\right) & =\left[Q_{Y}+p\left(1-\theta_{Y}\right)\right] \varepsilon_{Y} .
\end{aligned}
$$

By Proposition 6.4, each $u \in B_{p, p}^{\theta_{Y}}(\partial Y)$ extends to a function $U \in N^{1, p}(Y)$ so that the minimal upper gradient of $U$ satisfies

$$
\left\|g_{U}\right\|_{L^{p}(Y)} \lesssim\|u\|_{B_{p, p}^{\theta_{Y}(\partial Y)}} .
$$


Lemma 8.5 then shows that the function $V=U \circ F$ has an upper gradient $g \in L^{p}(X)$ with $\|g\|_{L^{p}(X)} \lesssim\left\|g_{U}\right\|_{L^{p}(Y)}$, provided that (8.9) holds. Moreover, the Poincaré inequality (4.4) applied with $z=0_{X}$ shows that $V \in L^{1}(X)$ and

$$
\left|V_{X}\right| \leq\left|V\left(0_{X}\right)\right|+C\|g\|_{L^{p}(X)} .
$$

By the construction of $F$ we know that $F\left(0_{X}\right)=0_{Y}$. Therefore we have

$$
\left|V\left(0_{X}\right)\right|=\left|U\left(0_{Y}\right)\right|=\left|f_{\partial Y} u d \nu_{Y}\right| \lesssim\|u\|_{L^{p}(\partial Y)} .
$$

Thus the $(p, p)$-Poincaré inequality in Corollary 4.3 gives that $V \in L^{p}(X)$ and $\|V\|_{L^{p}(X)} \lesssim\|u\|_{L^{p}(\partial Y)}+\|g\|_{L^{p}(X)}$. Hence $\|V\|_{N^{1, p}(X)} \lesssim\|u\|_{\widetilde{B}_{p, p}^{\theta_{Y}(\partial Y)}}$ and Proposition 6.1 then implies that $V$ has a trace $\operatorname{Tr} V$ on $\partial X$ such that $\|\operatorname{Tr} V\|_{\widetilde{B}_{p, p}^{\theta_{X}(\partial X)}} \lesssim$ $\|u\|_{\widetilde{B}_{p, p}^{\theta_{Y}(\partial Y)}} \cdot$

To show that (8.9) holds, note first that

$$
\beta_{X}-p \varepsilon_{X}=\varepsilon_{X}\left(Q_{X}-p \theta_{X}\right) \quad \text { and } \quad \beta_{Y}-p \varepsilon_{Y}=\varepsilon_{Y}\left(Q_{Y}-p \theta_{Y}\right) .
$$

Thus it suffices to show that

$$
\varepsilon_{X}\left(p \theta_{X}-Q_{X}\right)|x|+\varepsilon_{Y}\left(Q_{Y}-p \theta_{Y}\right)|F(x)| \leq C_{0}
$$

for some constant $C_{0}$. We need to distinguish two cases.

Case 1. If $\theta_{Y} \geq Q_{Y} / p$, then $Q_{Y}-p \theta_{Y} \leq 0$ and we use the fact that

$$
|F(x)| \geq\left|F(x)-F\left(0_{X}\right)\right|-\left|F\left(0_{X}\right)\right| \geq L_{1}|x|-\Lambda,
$$

since $F\left(0_{X}\right)=0_{Y}$. Hence, as $\theta_{X} \leq Q_{X} / p+\alpha_{1}\left(\theta_{Y}-Q_{Y} / p\right)$, the left-hand side of (8.12) is majorized by

$$
\alpha_{1} \varepsilon_{X}\left(p \theta_{Y}-Q_{Y}\right)|x|+\varepsilon_{Y}\left(Q_{Y}-p \theta_{Y}\right)\left(L_{1}|x|-\Lambda\right)=-\varepsilon_{Y}\left(Q_{Y}-p \theta_{Y}\right) \Lambda=: C_{0},
$$

since $L_{1}=\alpha_{1} \varepsilon_{X} / \varepsilon_{Y}$.

Case 2. If $\theta_{Y} \leq Q_{Y} / p$, then $Q_{Y}-p \theta_{Y} \geq 0$ and we use the fact that

$$
|F(x)| \leq\left|F\left(0_{X}\right)\right|+\left|F(x)-F\left(0_{X}\right)\right| \leq L_{2}|x|+\Lambda \text {. }
$$

Since $\theta_{X} \leq Q_{X} / p+\alpha_{2}\left(\theta_{Y}-Q_{Y} / p\right)$ and $L_{2}=\alpha_{2} \varepsilon_{X} / \varepsilon_{Y}$, we see as in Case 1 that (8.9) holds with $C_{0}=\varepsilon_{Y}\left(Q_{Y}-p \theta_{Y}\right) \Lambda$.

Finally, for continuous $u \in B_{p, p}^{\theta_{Y}}(\partial Y)$ we have, by construction,

$$
\operatorname{Tr} V(\zeta)=\lim _{X \ni x \rightarrow \zeta} V(x)=\lim _{Y \ni y \rightarrow f(\zeta)} U(x)=u \circ f(\zeta)
$$

\section{Quasisymmetric extensions of rough quasiisome- tries between trees}

In this section, we assume that both $X$ and $Y$ are rooted trees such that each vertex has at least one child. In particular, each branch is infinite, but no regularity is assumed.

Having shown that quasisymmetries between the boundaries extend to rough quasiisometries between the trees, we are next concerned with the issue of whether a rough quasiisometry between the trees can be extended to a quasisymmetry between the boundaries. For certain Gromov hyperbolic spaces (those whose boundaries are 
connected sets) this is known by the deep work of Gromov [20] and Kapovich [35], see also the exposition in Bourdon-Pajot [10]. Given the simple nature of trees, we are able to study this extendability question more directly here, but the fact that the boundaries of trees are totally disconnected means that it is not sufficient to merely check for "asymptotic" quasisymmetry. Also, quasisymmetric mappings may reverse the "order" of a triple of points; namely, $\xi$ can be closer to $\zeta$ than $\chi$, while the image of $\chi$ is closer to the image of $\zeta$ than the image of $\xi$ is. This fact makes our process of checking various cases rather troublesome, but is overcome with the help of Lemma 9.8, which says in essence that if such order reversal takes place, then $\xi$ and $\chi$ are relatively close. However, a careful accounting still must be taken.

We continue to use the same notation as in Section 8. Recall that any rough quasiisometry between trees, because of the attendant lack of control at small scales, can without loss of generality be thought of as a map solely between the vertices of the corresponding trees. This is the view we took in Theorem 8.2 and which we continue with in this section.

Let $F: X \rightarrow Y$ be an $(L, \Lambda)$-rough quasiisometry. We use the following construction to extend $F$ to a mapping between the boundaries $\partial X$ and $\partial Y$ : Given $\zeta \in \partial X$ let $\left\{x_{i}\right\}_{i=1}^{\infty}$ be the sequence of vertices in $X$ that form the geodesic connecting the root $x_{0}=0_{X}$ to $\zeta$ with $x_{i}$ being the parent of $x_{i+1}$ for $i \in \mathbb{N}$. Let $Y_{\zeta}=\left\{F\left(x_{i}\right): i \in \mathbb{N}\right\}$ be the collection of the images of the vertices in this sequence, and $\partial Y_{\zeta}:=\bar{Y}_{\zeta} \cap \partial Y$, where the closure is taken with respect to the $d_{Y}$ metric. Since $F$ is a rough quasiisometry we see that

$$
\left|F\left(x_{i}\right)\right| \geq\left|F\left(x_{i}\right)-F\left(0_{X}\right)\right|-\left|F\left(0_{X}\right)\right| \geq \frac{1}{L}\left|x_{i}\right|-\Lambda-\left|F\left(0_{X}\right)\right| \rightarrow \infty, \quad \text { as } i \rightarrow \infty,
$$

and thus $Y_{\zeta}$ is unbounded in the metric $|\cdot-\cdot|$. As all vertices in the tree $Y$ have finite degree, it is not hard to see that $\bar{Y}$ is compact, and thus $\partial Y_{\zeta}$ is nonempty.

Lemma 9.1 below shows that $\partial Y_{\zeta}$ has exactly one point, and thereby allows us to extend $F$ to the boundary $\partial X$ by letting $f(\zeta)=\chi$, where $\partial Y_{\zeta}=\{\chi\}$.

Lemma 9.1. Let $\zeta \in \partial X, \chi \in \partial Y_{\zeta}$, and $y \in Y$ be such that $y<\chi$. Let $\left\{x_{i}\right\}_{i=1}^{\infty}$ be the ordered sequence of vertices in $X$ forming the geodesic connecting $0_{X}$ to $\zeta$, whose image forms $Y_{\zeta}$. Then there is a positive integer $k$ such that whenever $x \in X$ satisfies $x \geq x_{k}$, we have $F(x) \geq y$ and hence $d_{Y}(F(x), \chi) \leq 2 e^{-\varepsilon_{Y}|y|} / \varepsilon_{Y}$. In particular, $\partial Y_{z}=\{\chi\}$.

Proof. The statement is trivial for $y=0_{Y}$. Assume therefore that $y \neq 0_{Y}$. Since $\chi$ is a limit point of $Y_{\zeta}$ and $y<\chi$, there exist positive integers $k$ and $j$ with $k>j$ such that $F\left(x_{j}\right), F\left(x_{k}\right) \geq y$ and

$$
\left|x_{k}-x_{j}\right| \geq L\left(L+2 \Lambda+1+\left|F\left(x_{j}\right)-y\right|\right)
$$

Now let $x \in X$ such that $x \geq x_{k}$. We need to show that $F(x) \geq y$. We do so by contradiction. Suppose $F(x) \nsupseteq y$. Then because $F\left(x_{k}\right) \geq y$, we can trace back from $x$ towards $x_{k}$ to find $z \in X$ with $x_{k}<z \leq x$ such that $F(z) \geq y$ but the parent $\hat{z}$ of $z$ satisfies $F(\hat{z}) \geq y$. Denoting the parent of $y$ by $\hat{y}$, we now see using (9.1) that

$$
\begin{aligned}
|F(z)-\hat{y}|+\left|\hat{y}-F\left(x_{j}\right)\right| & =\left|F(z)-F\left(x_{j}\right)\right| \geq \frac{1}{L}\left|z-x_{j}\right|-\Lambda \\
& \geq \frac{1}{L}\left|x_{k}-x_{j}\right|-\Lambda \geq L+\Lambda+1+\left|F\left(x_{j}\right)-y\right| .
\end{aligned}
$$

Given that $F\left(x_{j}\right) \geq y$ and so $\left|\hat{y}-F\left(x_{j}\right)\right|=1+\left|y-F\left(x_{j}\right)\right|$, we obtain

$$
|F(z)-\hat{y}| \geq L+\Lambda \text {. }
$$


On the other hand, since the geodesic from $F(z)$ to $F(\hat{z})$ must pass through $\hat{y}$, we have $|F(z)-\hat{y}|+|\hat{y}-F(\hat{z})|=|F(z)-F(\hat{z})| \leq L+\Lambda$, and given that $F(\hat{z}) \geq y$, we know that $|\hat{y}-F(\hat{z})| \geq 1$. Therefore

$$
|F(z)-\hat{y}| \leq L+\Lambda-1
$$

which contradicts (9.2). Thus, for $x \geq x_{k}$ we have $F(x) \geq y$ and hence $d_{Y}(F(x), \chi) \leq$ $2 e^{-\varepsilon_{Y}|y|} / \varepsilon_{Y}$.

Letting $y \rightarrow \chi$ along the geodesic from $0_{Y}$ to $\chi$ now shows that $F\left(x_{j}\right) \rightarrow \chi$ when $x_{j} \rightarrow \zeta$ along the geodesic from $0_{X}$ to $\zeta$.

The next goal is to show that $f: \partial X \rightarrow \partial Y$ is a bijection and a homeomorphism. This will be done through several lemmas, some of which (Lemmas 9.3 and 9.6) may be of independent interest.

Lemma 9.2. The mapping $f$ is surjective.

Proof. Let $\omega \in \partial Y$ be arbitrary and let $\left\{y_{j}\right\}_{j=0}^{\infty}$ be a geodesic terminating at $\omega$. By the density condition, for every $j=0,1, \ldots$, there exists $x_{j} \in X$ such that $\left|F\left(x_{j}\right)-y_{j}\right| \leq L+\Lambda$. Then $F\left(x_{j}\right) \rightarrow \omega$ as $j \rightarrow \infty$. By compactness, there is a subsequence (also denoted $\left\{x_{j}\right\}_{j=0}^{\infty}$ ) converging in the metric $d_{X}$ to some $\zeta \in \bar{X}$. Since

$$
\left|x_{j}\right|=\left|x_{j}-0_{X}\right| \geq \frac{\left|F\left(x_{j}\right)-F\left(0_{X}\right)\right|-\Lambda}{L} \rightarrow \infty \quad \text { as } j \rightarrow \infty,
$$

we see that $\zeta \in \partial X$.

Letting $\hat{x}_{j}$ be the largest common ancestor of $\left\{x_{i}\right\}_{i=j}^{\infty}$, we have that $\hat{x}_{j} \rightarrow \zeta$ along a geodesic from $0_{X}$ to $\zeta$. Hence $F\left(\hat{x}_{j}\right) \in Y_{\zeta}$ and $F\left(\hat{x}_{j}\right) \rightarrow f(\zeta)$. Lemma 9.1 applied to $y=\hat{y}_{k}$, where $\hat{y}_{k}$ is the largest common ancestor of $F\left(\hat{x}_{k}\right)$ and $f(\zeta)$, then shows that $F\left(x_{j}\right) \geq \hat{y}_{k}$ for all sufficiently large $j$. Thus $d_{Y}\left(F\left(x_{j}\right), f(\zeta)\right) \leq 2 e^{\varepsilon_{y}\left|\hat{y}_{k}\right|} / \varepsilon_{Y} \rightarrow 0$ as $k \rightarrow \infty$, showing that $f(\zeta)=\omega$.

The proof of the main result of this section needs the following Morse-type lemma.

Lemma 9.3. Let $\gamma$ be a geodesic in $X$ connecting $x_{0} \in X$ to $\zeta \in \partial X$, and $\gamma^{\prime}$ be the geodesic in $Y$ connecting $F\left(x_{0}\right)$ to $f(\zeta) \in \partial Y$. Let $\tau>(L+\Lambda)\left(2 L^{2}+3 \Lambda L+1\right)$ and $\tau^{\prime}>L+\Lambda$. Then

$$
F(\gamma) \subset \bigcup_{y \in \gamma^{\prime}} B_{Y}(y, \tau) \quad \text { and } \quad \gamma^{\prime} \subset \bigcup_{x \in \gamma} B_{Y}\left(F(x), \tau^{\prime}\right),
$$

where $B_{Y}$ denotes open balls in $Y$ with respect to the metric $|\cdot-\cdot|_{Y}$. In particular, the Hausdorff distance $\operatorname{dist}_{H}\left(F(\gamma), \gamma^{\prime}\right)<\tau$.

The proof of this lemma is a straightforward modification of the one found in Kapovich [35, Lemma 3.43] and employs the following simple projection lemma.

Lemma 9.4. Let $F$ and $F^{\prime}$ be two disjoint nonempty pathconnected closed subsets of a tree $Y$, and $\operatorname{proj}_{F}: Y \rightarrow F$ be the nearest point projection, that is, for each $y \in Y$, the point $\operatorname{proj}_{F}(y) \in F$ is such that $\operatorname{dist}(y, F)=\left|y-\operatorname{proj}_{F}(y)\right|$. Then

(a) $\operatorname{proj}_{F}$ is well defined;

(b) the set $\operatorname{proj}_{F}\left(F^{\prime}\right)$ has exactly one element.

Proof. We start with part (b). Let $x_{1}, x_{2} \in F^{\prime}$. Then for $j=1,2$ there is $z_{j} \in F$ with $\operatorname{dist}\left(x_{j}, F\right)=\left|z_{j}-x_{j}\right|$. The geodesic from $x_{j}$ to $z_{j}$ has a last vertex $y_{j} \in F^{\prime}$ and we let $\gamma_{j}$ be the geodesic from $y_{j}$ to $z_{j}$. It only hits $F^{\prime}$ at $y_{j}$ and $F$ at $z_{j}$. Moreover, let $\gamma$ be the geodesic in $F^{\prime}$ from $y_{1}$ to $y_{2}$, and $\varphi$ be the geodesic in $F$ from $z_{2}$ to $z_{1}$. Then the concatenation of $\gamma, \gamma_{2}$ and $\varphi$ is a geodesic from $y_{1}$ to $z_{1}$, which must coincide with $\gamma_{1}$ as $Y$ is a tree, and in particular $z_{1}=z_{2}$. This completes the proof of (b). Part (a) follows by letting $x=x_{1}=x_{2}$. 
Proof of Lemma 9.3. Let $\left\{x_{i}\right\}_{i=0}^{\infty}$ be the sequence of vertices in $X$ representing $\gamma$ so that $x_{i} \rightarrow \zeta$ as $i \rightarrow \infty$. For each $i$ let $\gamma_{i}$ be the geodesic in $Y$ connecting $F\left(x_{i}\right)$ to $F\left(x_{i+1}\right)$, and let $\varphi=\sum_{i=0}^{\infty} \gamma_{i}$ be the concatenation of these geodesics. Because $F$ is a rough quasiisometry, we know that

$$
F(\gamma) \subset \varphi \subset \bigcup_{i=0}^{\infty} B_{Y}\left(F\left(x_{i}\right), \tau^{\prime}\right) .
$$

Since $\gamma^{\prime} \subset \varphi$ this proves the second inclusion. Thus it suffices to show that $\varphi \subset$ $\bigcup_{y \in \gamma^{\prime}} B_{Y}(y, \tau)$. Since $F\left(x_{i}\right) \rightarrow f(\zeta)$, we can find a subsequence $\left\{x_{i_{k}}\right\}_{k=1}^{\infty}$ such that

$$
d_{Y}\left(F\left(x_{i_{k+1}}\right), f(\zeta)\right)<\frac{1}{2} d_{Y}\left(F\left(x_{i_{k}}\right), f(\zeta)\right)<\frac{1}{2} d_{Y}\left(F\left(x_{0}\right), f(\zeta)\right)
$$

for each positive integer $k$. Let $y_{i}$ be the largest common ancestor of $F\left(x_{i}\right)$ and $f(\zeta), i=0,1, \ldots$. Then

$$
\frac{1}{2} d_{Y}\left(F\left(x_{i}\right), f(\zeta)\right)<d_{Y}\left(y_{i}, f(\zeta)\right) \leq d_{Y}\left(F\left(x_{i}\right), f(\zeta)\right), \quad i=0,1, \ldots
$$

and thus

$$
d_{Y}\left(y_{i_{k+1}}, f(\zeta)\right)<d_{Y}\left(y_{i_{k}}, f(\zeta)\right)<d_{Y}\left(y_{0}, f(\zeta)\right) .
$$

It follows that both $y_{i_{k}}$ and $y_{i_{k+1}}$ belong to $\gamma^{\prime}$, and also that the geodesic connecting $F\left(x_{i_{k}}\right)$ to $F\left(x_{i_{k+1}}\right)$ contains $y_{i_{k}}$. This geodesic is contained in the path $\sum_{i=i_{k}}^{i_{k+1}-1} \gamma_{i}$ between $F\left(x_{i_{k}}\right)$ and $F\left(x_{i_{k+1}}\right)$, and thus $y_{i_{k}} \in \gamma_{j_{k}}$ for some $j_{k}$ with $i_{k} \leq j_{k}<i_{k+1}$.

Suppose next that a subpath $\varphi^{\prime}:=\sum_{k=i}^{j-1} \gamma_{k}$ of $\varphi$, with $j>i$, does not intersect $\gamma^{\prime}$. Because each $\gamma_{j_{k}}, k=1,2, \ldots$, intersect $\gamma^{\prime}$, we can choose $\varphi^{\prime}$ so that both $\gamma_{i-1}$ and $\gamma_{j}$ intersect $\gamma^{\prime}$. Since $F$ is a rough quasiisometry, both $\gamma_{i-1}$ and $\gamma_{j}$ have length at most $L+\Lambda$. We can thus conclude that both $F\left(x_{i}\right)$ and $F\left(x_{j}\right)$ lie within the closed $(L+\Lambda)$-neighborhood of $\gamma^{\prime}$. By the rough quasiisometry again, we know that

$$
\left|F\left(x_{i}\right)-F\left(x_{j}\right)\right| \geq \frac{\left|x_{i}-x_{j}\right|}{L}-\Lambda=\frac{j-i}{L}-\Lambda .
$$

By Lemma 9.4, we know that $\operatorname{proj}_{\gamma^{\prime}}\left(\varphi^{\prime}\right)=\{a\}$ for some $a \in \gamma^{\prime}$, and hence

$$
\begin{aligned}
& \left|F\left(x_{i}\right)-a\right|=\operatorname{dist}\left(F\left(x_{i}\right), \gamma^{\prime}\right) \leq L+\Lambda, \\
& \left|F\left(x_{j}\right)-a\right|=\operatorname{dist}\left(F\left(x_{j}\right), \gamma^{\prime}\right) \leq L+\Lambda .
\end{aligned}
$$

It follows that $\left|F\left(x_{i}\right)-F\left(x_{j}\right)\right| \leq 2(L+\Lambda)$, and inserting this into (9.3) yields $j-i \leq L(2 L+3 \Lambda)$. Using that $\left|F\left(x_{k}\right)-F\left(x_{k+1}\right)\right| \leq L+\Lambda$ for all $k$, we find that

$$
\ell\left(\varphi^{\prime}\right) \leq(j-i)(L+\Lambda) \leq L(L+\Lambda)(2 L+3 \Lambda) .
$$

Together with (9.4) this shows the first inclusion.

Lemma 9.5. The mapping $f$ is injective.

Proof. Suppose that there are $\zeta_{1}, \zeta_{2} \in \partial X$ with $\zeta_{1} \neq \zeta_{2}$ such that $f\left(\zeta_{1}\right)=f\left(\zeta_{2}\right)=\chi$. Let $\hat{x}$ be the largest common ancestor of $\zeta_{1}$ and $\zeta_{2}$. By Lemma 9.3, the geodesic from $F(\hat{x})$ to $\chi$ lies within $\tau$-neighborhoods of both $Y_{\zeta_{1}}$ and $Y_{\zeta_{2}}$. It follows that $Y_{\zeta_{1}}$ and $Y_{\zeta_{2}}$ lie within $2 \tau$-neighborhoods of each other. Thus, for each $x_{k}$ in the geodesic from $\hat{x}$ to $\zeta_{1}$, there is $x_{k}^{\prime}$ in the geodesic from $\hat{x}$ to $\zeta_{2}$ such that $\left|F\left(x_{k}\right)-F\left(x_{k}^{\prime}\right)\right| \leq 2 \tau$. This gives

$$
\left|x_{k}-x_{k}^{\prime}\right| \leq L\left(\left|F\left(x_{k}\right)-F\left(x_{k}^{\prime}\right)\right|+\Lambda\right) \leq L(2 \tau+\Lambda) .
$$

On the other hand, $\left|x_{k}-x_{k}^{\prime}\right| \geq\left|x_{k}-\hat{x}\right| \rightarrow \infty$, as $x_{k} \rightarrow \zeta_{1}$, giving a contradiction. 
Hence now we know that $f: \partial X \rightarrow \partial Y$ is a bijective mapping. To show that it is a homeomorphism, one can use Lemma 9.1 to prove continuity of $f$, and then the compactness of $\partial X$ and $\partial Y$ gives the continuity of $f^{-1}$. We shall instead in Lemma 9.7 below improve upon this and show that $f$ is biHölder continuous. The following useful lemma is a rather simple consequence of Lemma 9.3.

Lemma 9.6. Let $x \in X$ be the largest common ancestor of $\zeta, \xi \in \partial X, \zeta \neq \xi$, and $y \in Y$ be the largest common ancestor of $f(\zeta)$ and $f(\xi)$. Then $|F(x)-y| \leq C(L, \Lambda)$.

Proof. By Lemma 9.3, the geodesics from $y$ to $f(\zeta)$ and to $f(\xi)$ are within $\tau^{\prime}$ neighborhoods of the images of the geodesics from $x$ to $\zeta$ and to $\xi$, respectively. It follows that there are vertices $a, b \in X$ such that $x \leq a<\zeta, x \leq b<\xi$,

$$
|F(a)-y| \leq \tau^{\prime} \quad \text { and } \quad|F(b)-y| \leq \tau^{\prime}
$$

Hence, as $F$ is a rough quasiisometry, we have

$$
|x-a| \leq|b-a| \leq L(|F(b)-F(a)|+\Lambda) \leq L\left(2 \tau^{\prime}+\Lambda\right)
$$

and consequently, using (9.5) again,

$$
\begin{aligned}
|F(x)-y| & \leq|F(x)-F(a)|+|F(a)-y| \\
& \leq L|x-a|+\Lambda+\tau^{\prime} \leq L^{2}\left(2 \tau^{\prime}+\Lambda\right)+\Lambda+\tau^{\prime} .
\end{aligned}
$$

Lemma 9.7. The mapping $f: \partial X \rightarrow \partial Y$ is $\left(\alpha_{2}, \alpha_{1}\right)$-biHölder continuous with

$$
\alpha_{1}=\frac{L_{1} \varepsilon_{Y}}{\varepsilon_{X}} \quad \text { and } \quad \alpha_{2}=\frac{L_{2} \varepsilon_{Y}}{\varepsilon_{X}},
$$

that is,

$$
C_{1} d_{Z}(x, y)^{\alpha_{2}} \leq d_{W}(f(x), f(y)) \leq C_{2} d_{Z}(x, y)^{\alpha_{1}},
$$

where the constants $C_{1}$ and $C_{2}$ depend only on $L_{1}, L_{2}, \Lambda,\left|F\left(0_{X}\right)\right|, \varepsilon_{X}$ and $\varepsilon_{Y}$.

Proof. Given $\zeta, \xi \in \partial X$ with $\zeta \neq \xi$, let $x$ be the largest common ancestor of $\zeta$ and $\xi$. Similarly, let $y$ denote the largest common ancestor of $f(\zeta)$ and $f(\xi)$. We shall estimate $d_{Y}(f(\zeta), f(\xi)) \simeq e^{-\varepsilon_{Y}|y|}$ in terms of $d_{X}(\zeta, \xi) \simeq e^{-\varepsilon_{X}|x|}$.

Lemma 9.6 implies that $|F(x)-y| \leq C$ and hence $e^{-\varepsilon_{Y}|y|} \simeq e^{-\varepsilon_{Y}|F(x)|}$. At the same time, the rough quasiisometry property (8.3) gives

$$
|F(x)| \leq\left|F(x)-F\left(0_{X}\right)\right|+\left|F\left(0_{X}\right)\right| \leq L_{2}|x|+\Lambda+\left|F\left(0_{X}\right)\right|
$$

and

$$
|F(x)| \geq\left|F(x)-F\left(0_{X}\right)\right|-\left|F\left(0_{X}\right)\right| \geq L_{1}|x|-\Lambda-\left|F\left(0_{X}\right)\right| .
$$

From this we conclude that

$$
d_{Y}(f(\zeta), f(\xi)) \simeq e^{-\varepsilon_{Y}|F(x)|} \gtrsim e^{-L_{2} \varepsilon_{Y}|x|} \simeq d_{X}(\zeta, \xi)^{L_{2} \varepsilon_{Y} / \varepsilon_{X}}
$$

and

$$
d_{Y}(f(\zeta), f(\xi)) \simeq e^{-\varepsilon_{Y}|F(x)|} \lesssim e^{-L_{1} \varepsilon_{Y}|x|} \simeq d_{X}(\zeta, \xi)^{L_{1} \varepsilon_{Y} / \varepsilon_{X}} .
$$

This gives the biHölder condition (7.4) with $\alpha_{1}$ and $\alpha_{2}$ as in (9.6). 
Lemma 9.8. Let $\zeta, \xi, \chi \in \partial X$ be such that $0<d_{X}(\zeta, \xi)<d_{X}(\zeta, \chi)$ and assume that

$$
d_{X}(\zeta, \chi) \leq\left(\frac{C_{1}}{C_{2}}\left(\frac{1}{3} \operatorname{diam} \partial X\right)^{\alpha_{2}}\right)^{1 / \alpha_{1}}=: r_{0},
$$

where $\alpha_{1}, \alpha_{2}, C_{1}$ and $C_{2}$ are as in Lemma 9.7. Let $x \in X$ be the largest common ancestor of $\zeta$ and $\xi$, and let $y \in X$ be the largest common ancestor of $\zeta$ and $\chi$. If $|x-y| \geq L(3 C(L, \Lambda)+2 \Lambda+L)=: s_{0}$, where $C(L, \Lambda)$ is as in Lemma 9.6, then $d_{Y}(f(\zeta), f(\xi)) \leq d_{Y}(f(\zeta), f(\chi))$.

To prove this we will use the following obvious fact: If $u, v, w \in Y$ satisfy $|u-v| \geq|w-v|$ and $u \leq v$, then $u \leq w$.

Proof. Suppose that $d_{Y}(f(\zeta), f(\xi))>d_{Y}(f(\zeta), f(\chi))$. Let $x_{1} \in Y$ be the largest common ancestor of $f(\zeta)$ and $f(\xi)$, and $y_{1}$ be the largest common ancestor of $f(\zeta)$ and $f(\chi)$. Note that $x_{1}<y_{1}$. By Lemma 9.6 we know that $\left|F(x)-x_{1}\right| \leq C(L, \Lambda)$ and $\left|F(y)-y_{1}\right| \leq C(L, \Lambda)$. Therefore by the rough quasiisometry of $F$,

$$
\begin{aligned}
\left|x_{1}-y_{1}\right| & \geq|F(x)-F(y)|-\left|F(x)-x_{1}\right|-\left|F(y)-y_{1}\right| \\
& \geq \frac{1}{L}|x-y|-\Lambda-2 C(L, \Lambda) \geq C(L, \Lambda)+\Lambda+L .
\end{aligned}
$$

Since $x_{1}<y_{1}$ and $\left|F(y)-y_{1}\right| \leq C(L, \Lambda) \leq\left|x_{1}-y_{1}\right|$, our remark before the proof shows that $x_{1} \leq F(y)$.

Now let $\omega \in \partial X$ be such that $d_{X}(\zeta, \omega) \geq \max \left\{\frac{1}{3} \operatorname{diam} \partial X, d_{X}(\zeta, \chi)\right\}$. Let $z$ be the largest common ancestor of $\zeta$ and $\omega$, and $z_{1}$ be the largest common ancestor of $f(\zeta)$ and $f(\omega)$. Note that $z \leq y \leq x$. Then by Lemma 9.7 and (9.7) we have that

$$
d_{Y}(f(\zeta), f(\omega)) \geq C_{1} d_{X}(\zeta, \omega)^{\alpha_{2}} \geq C_{2} d_{X}(\zeta, \chi)^{\alpha_{1}}>C_{2} d_{X}(\zeta, \xi)^{\alpha_{1}} \geq d_{Y}(f(\zeta), f(\xi))
$$

from which it follows that $z_{1}<x_{1}$. As in (9.8) we have

$$
\left|x_{1}-z_{1}\right| \geq \frac{1}{L}|x-z|-\Lambda-2 C(L, \Lambda) \geq C(L, \Lambda)+\Lambda+L .
$$

Suppose that $F(z) \geq x_{1}$. Then by Lemma 9.6,

$$
C(L, \Lambda) \geq\left|F(z)-z_{1}\right| \geq\left|x_{1}-z_{1}\right| \geq C(L, \Lambda)+\Lambda+L,
$$

which is not possible. Hence $F(z) \nsupseteq x_{1}$. Since $F(y) \geq x_{1}$, we can find $p \in X$ and its parent $\hat{p}$ with $z \leq \hat{p}<p \leq y \leq x$ such that $F(p) \geq x_{1}$ and $F(\hat{p}) \geq x_{1}$. It follows that

$$
\left|F(p)-x_{1}\right|<|F(p)-F(\hat{p})| \leq L+\Lambda \text {. }
$$

This leads to a contradiction because by Lemma 9.6 again,

$$
\begin{aligned}
L+\Lambda+C(L, \Lambda) & >\left|F(p)-x_{1}\right|+\left|F(x)-x_{1}\right| \geq|F(p)-F(x)| \\
& \geq \frac{1}{L}|p-x|-\Lambda \geq \frac{1}{L}|y-x|-\Lambda \geq 3 C(L, \Lambda)+\Lambda+L,
\end{aligned}
$$

which is not possible. Thus the assumption that $d_{Y}(f(\zeta), f(\xi))>d_{Y}(f(\zeta), f(\chi))$ is false, and the lemma is proved.

Finally, we are ready to prove the main result of this section.

Theorem 9.9. If the rough quasiisometry $F: X \rightarrow Y$ satisfies

$$
L_{1}\left|x_{1}-x_{2}\right|-\Lambda \leq\left|F\left(x_{1}\right)-F\left(x_{2}\right)\right| \leq L_{2}\left|x_{1}-x_{2}\right|+\Lambda \quad \text { for all } x_{1}, x_{2} \in X,
$$

then the mapping $f: \partial X \rightarrow \partial Y$ is an $\eta$-quasisymmetric map, where

$$
\eta(t)=\left\{\begin{array}{ll}
A t^{\alpha_{1}}, & \text { if } t \leq 1, \\
A t^{\alpha_{2}}, & \text { if } t \geq 1,
\end{array} \quad \alpha_{1}=\frac{L_{1} \varepsilon_{Y}}{\varepsilon_{X}}, \quad \alpha_{2}=\frac{L_{2} \varepsilon_{Y}}{\varepsilon_{X}} \quad \text { and } \quad A>0 .\right.
$$


Remark 9.10. Suppose that each vertex in $X$ and $Y$ has at least two children. Let $f: \partial X \rightarrow \partial Y$ be an $\eta$-quasisymmetric map with $\eta$ as in (9.9). If we first extend it to a rough quasiisometry $F: X \rightarrow Y$ using Theorem 8.2 and then apply Theorem 9.9 we get $f$ back with the same exponents $\alpha_{1}$ and $\alpha_{2}$. This shows that the formulas for $\alpha_{1}$ and $\alpha_{2}$ in Theorem 9.9 as well as the formulas for $L_{1}$ and $L_{2}$ in Theorem 8.2 are all sharp.

Similarly, if $F: X \rightarrow Y$ is an $(L, \Lambda)$-rough quasiisometry, then Theorem 9.9 gives us a quasisymmetry $f: \partial X \rightarrow \partial Y$, which in turn, by Theorem 8.2, induces an $\left(L, \Lambda^{\prime}\right)$-rough quasiisometry $G: X \rightarrow Y$ (called $F$ in Section 8) with a better behavior than the original map $F$. By construction, $G\left(0_{X}\right)=0_{Y}$ and $G$ is orderpreserving, i.e. $G(x) \leq G(y)$ whenever $x \leq y$. Moreover, for every $x \in X$ we have $|F(x)-G(x)| \leq \tau$ for some $\tau$. To see this, let $x \in X$ be arbitrary and let $\zeta, \chi \in \partial X$ be such that $\zeta, \chi>x$ and $f(\zeta)$ and $f(\chi)$ are descendants of two distinct children of $G(x)$, which is possible by the construction of $G$. Let $z \geq x$ be the largest common ancestor of $\zeta$ and $\chi$. Since $x$ has at least two children, there exists $\xi \in \partial X$ such that $x$ is the largest common ancestor of $\zeta$ and $\xi$. Because $\xi>x$ and $G$ is orderpreserving (and induces $f$ ), we get that $f(\xi)>G(x)$. Therefore $d_{Y}(f(\zeta), f(\chi)$ ) equals either $d_{Y}(f(\zeta), f(\xi))$ or $d_{Y}(f(\chi), f(\xi))$. In the first case we have, as $f$ is an $\eta$-quasisymmetry, that

$$
1=\frac{d_{Y}(f(\zeta), f(\chi))}{d_{Y}(f(\zeta), f(\xi))} \leq \eta\left(\frac{d_{X}(\zeta, \chi)}{d_{X}(\zeta, \xi)}\right)=\eta\left(e^{\varepsilon_{X}(|x|-|z|)}\right),
$$

which yields $|x-z|=|z|-|x| \leq \tau_{0}:=\left(\log \eta^{-1}(1)\right) / \varepsilon_{X}$. In the second case, a similar argument with the roles of $\zeta$ and $\chi$ interchanged gives $|x-z| \leq \tau_{0}$. Thus, in either case we have $|F(x)-F(z)| \leq L \tau_{0}+\Lambda$. Together with the estimate $|F(z)-G(x)| \leq C(L, \Lambda)$ of Lemma 9.6, this gives $|F(x)-G(x)| \leq \tau$.

Theorem 9.9, for more general Gromov hyperbolic spaces, seems to have been stated in Bourdon-Pajot [10], where the credit for it is given to Gromov [20]. However, we were not able to find this result in [20], and so we give a self-contained proof here. Our proof of Theorem 9.9 uses tools inspired by the proof of Kapovich [35, Theorem 3.47]. Similar ideas can be found in Jeffers [32] for a result characterizing isometries of $\mathbb{H}^{n}$.

Proof of Theorem 9.9. Let $\zeta, \xi, \chi \in \partial X$ be such that $\chi \neq \zeta \neq \xi$, and let

$$
t=\frac{d_{X}(\zeta, \xi)}{d_{X}(\zeta, \chi)}
$$

Assume first that $d_{X}(\zeta, \xi) \leq r_{0}$ and $d_{X}(\zeta, \chi) \leq r_{0}$, where $r_{0}$ is as in Lemma 9.8. Adopting the notation from Lemma 9.8 we let $x, y \in X$ and $x_{1}, y_{1} \in Y$ be the largest common ancestors of the pairs $\zeta$ and $\xi, \zeta$ and $\chi, f(\zeta)$ and $f(\xi)$, and $f(\zeta)$ and $f(\chi)$, respectively. By Lemma 9.6 and the rough quasiisometry of $F$,

$$
\begin{aligned}
\left|x_{1}-y_{1}\right| & \geq|F(x)-F(y)|-\left|F(x)-x_{1}\right|-\left|F(y)-y_{1}\right| \\
& \geq L_{1}|x-y|-\Lambda-2 C(L, \Lambda),
\end{aligned}
$$

where $L=\max \left\{1 / L_{1}, L_{2}\right\}$, and similarly,

$$
\left|x_{1}-y_{1}\right| \leq|F(x)-F(y)|+\left|F(x)-x_{1}\right|+\left|F(y)-y_{1}\right| \leq L_{2}|x-y|+\Lambda+2 C(L, \Lambda) .
$$

We have $t=e^{-\varepsilon_{X}(|x|-|y|)}$. With $t_{0}=e^{-\varepsilon_{X} s_{0}}<1$ and $t_{1}=1 / t_{0}=e^{\varepsilon_{X} s_{0}}>1$, where $s_{0}=L(3 C(L, \Lambda)+2 \Lambda+L)$ is as in Lemma 9.8, we consider three cases. 
Case A. $0<t \leq t_{0}$. In this case $x>y$ and $|x-y| \geq s_{0}$. So by Lemma $9.8, x_{1} \geq y_{1}$. Thus by (9.10),

$$
\frac{d_{Y}(f(\zeta), f(\xi))}{d_{Y}(f(\zeta), f(\chi))}=e^{-\varepsilon_{Y}\left(\left|x_{1}\right|-\left|y_{1}\right|\right)}=e^{-\varepsilon_{Y}\left|x_{1}-y_{1}\right|} \lesssim e^{-\varepsilon_{Y} L_{1}|x-y|}=t^{L_{1} \varepsilon_{Y} / \varepsilon_{X}} .
$$

Case B. $t \geq t_{1}$. In this case $x<y$ and $|x-y| \geq s_{0}$. So by Lemma 9.8, applied with the roles of $\xi$ and $\chi$ swapped, we see that $x_{1} \leq y_{1}$. Thus by (9.11),

$$
\frac{d_{Y}(f(\zeta), f(\xi))}{d_{Y}(f(\zeta), f(\chi))}=e^{-\varepsilon_{Y}\left(\left|x_{1}\right|-\left|y_{1}\right|\right)}=e^{\varepsilon_{Y}\left|x_{1}-y_{1}\right|} \lesssim e^{\varepsilon_{Y} L_{2}|x-y|}=t^{L_{2} \varepsilon_{Y} / \varepsilon_{X}} .
$$

Case C. $t_{0} \leq t \leq t_{1}$. In this case $|x-y| \leq s_{0}$ and thus by (9.11), $\left|x_{1}-y_{1}\right| \leq$ $L_{2} s_{0}+\Lambda+2 C(L, \Lambda)$, from which it follows that

$$
\frac{d_{Y}(f(\zeta), f(\xi))}{d_{Y}(f(\zeta), f(\chi))}=e^{-\varepsilon_{Y}\left(\left|x_{1}\right|-\left|y_{1}\right|\right)} \leq e^{\varepsilon_{Y}\left|x_{1}-y_{1}\right|} \lesssim 1
$$

We have thus shown that if $\zeta, \xi, \chi \in X, \zeta \neq \xi \neq \chi, d_{X}(\zeta, \xi) \leq r_{0}$ and $d_{X}(\zeta, \chi) \leq r_{0}$, then

$$
\frac{d_{Y}(f(\zeta), f(\xi))}{d_{Y}(f(\zeta), f(\chi))} \leq \tilde{\eta}\left(\frac{d_{X}(\zeta, \xi)}{d_{X}(\zeta, \chi)}\right), \quad \text { where } \tilde{\eta}(t) \simeq \begin{cases}t^{L_{1} \varepsilon_{Y} / \varepsilon_{X}}, & \text { if } 0 \leq t \leq t_{0} \\ 1, & \text { if } t_{0}<t<t_{1} \\ t^{L_{2} \varepsilon_{Y} / \varepsilon_{X}}, & \text { if } t \geq t_{1}\end{cases}
$$

and the comparison constant depends only on $L$ and $\Lambda$.

Assume now that $d_{X}(\zeta, \xi) \geq r_{0}$ or $d_{X}(\zeta, \chi) \geq r_{0}$. We shall again distinguish three cases. Note that $r_{0} \simeq \operatorname{diam} \partial X$.

Case 1. $d_{X}(\zeta, \xi) \leq r_{0} \leq d_{X}(\zeta, \chi) \leq \operatorname{diam} \partial X$. Then $t \leq 1$ and by Lemma 9.7,

$$
\frac{d_{Y}(f(\zeta), f(\xi))}{d_{Y}(f(\zeta), f(\chi))} \leq \frac{C_{2} d_{X}(\zeta, \xi)^{\alpha_{1}}}{C_{1} r_{0}^{\alpha_{2}}} \lesssim\left(\frac{d_{X}(\zeta, \xi)}{d_{X}(\zeta, \chi)}\right)^{\alpha_{1}} \lesssim \tilde{\eta}\left(\frac{d_{X}(\zeta, \xi)}{d_{X}(\zeta, \chi)}\right)
$$

Case 2. $\operatorname{diam} \partial X \geq d_{X}(\zeta, \xi) \geq r_{0} \geq d_{X}(\zeta, \chi)$. Then $t \geq 1$ and by Lemma 9.7,

$$
\frac{d_{Y}(f(\zeta), f(\xi))}{d_{Y}(f(\zeta), f(\chi))} \leq \frac{\operatorname{diam} \partial Y}{C_{1} d_{X}(\zeta, \chi)^{\alpha_{2}}} \lesssim\left(\frac{d_{X}(\zeta, \xi)}{d_{X}(\zeta, \chi)}\right)^{\alpha_{2}} \lesssim \tilde{\eta}\left(\frac{d_{X}(\zeta, \xi)}{d_{X}(\zeta, \chi)}\right) .
$$

Case 3. $r_{0} \leq d_{X}(\zeta, \xi) \leq \operatorname{diam} \partial X$ and $r_{0} \leq d_{X}(\zeta, \chi) \leq \operatorname{diam} \partial X$. Then $t \simeq 1$ and by Lemma 9.7 again,

$$
\frac{d_{Y}(f(\zeta), f(\xi))}{d_{Y}(f(\zeta), f(\chi))} \simeq 1 \simeq \frac{d_{X}(\zeta, \xi)}{d_{X}(\zeta, \chi)} \lesssim \tilde{\eta}\left(\frac{d_{X}(\zeta, \xi)}{d_{X}(\zeta, \chi)}\right)
$$

Thus there is $A^{\prime}$ such that

$$
\frac{d_{Y}(f(\zeta), f(\xi))}{d_{Y}(f(\zeta), f(\chi))} \leq A^{\prime} \tilde{\eta}\left(\frac{d_{X}(\zeta, \xi)}{d_{X}(\zeta, \chi)}\right)
$$

for all $\zeta, \xi, \chi \in \partial X$ with $\chi \neq \zeta \neq \xi$. A homeomorphism $\eta:[0, \infty) \rightarrow[0, \infty)$ of the form (9.9) such that $\eta \geq A^{\prime} \tilde{\eta}$, shows that $f$ is $\eta$-quasisymmetric.

We conclude this paper by considering a Mostow-type rigidity result. The setting of trees where the edges are hyperbolic regions pasted together in a combinatorial way was studied in Bourdon-Pajot [9]. It was shown in [9] that if there is a rough quasiisometry (called a quasiisometry in [9]) between two such hyperbolic trees (called hyperbolic buildings there), then that rough quasiisometry is a bounded 
distance from an isometry between the trees; in particular, the two hyperbolic trees, if rough quasiisometrically equivalent, are necessarily isometric. Their proof needs the boundaries of hyperbolic trees to be connected (and in fact to support a Poincaré inequality). In contrast, in our setting the boundaries of the two trees are totally disconnected, and support no Poincaré inequality.

There are many other rigidity theorems of various types in geometry. It is shown in Beardon-Minda [1] and Jeffers [32] that any bijective self-map of the hyperbolic space or the Euclidean space must be an isometry if it preserves complete geodesics. Given that trees are naturally Gromov hyperbolic, it is natural to ask similar questions in our setting. We show below that if an injective and almost surjective map between trees maps geodesics into geodesics, then it is an isometry. As Example 9.12 below shows, in general we have no rigidity theorem of the Bourdon-Pajot type for rough quasiisometries.

Proposition 9.11. Let $X$ and $Y$ be two rooted trees such that each vertex has at least two children. Assume that $G: X \rightarrow Y$ satisfies the following assumptions:

(a) $G$ is injective;

(b) $G$ maps geodesics into geodesics;

(c) for each $y \in Y$ there is $x \in X$ such that $G(x)>y$;

(d) $G\left(0_{X}\right)=0_{Y}$ or $0_{Y}$ has at least three children.

Then $G$ is an isometry (with respect to $|\cdot-\cdot|$ ).

Note that Condition (c) is satisfied by a rough quasiisometry $F: X \rightarrow Y$ because of the density property $\operatorname{dist}_{H}(Y, F(X))<\infty$.

Here we say that a mapping maps geodesics into geodesics if every geodesic line (that is, a geodesic connecting two boundary points) in $X$ gets mapped into a geodesic line in $Y$ (but not necessarily onto). Note that each geodesic path [x,y] in a tree lies inside a geodesic line, but we do not require its image to lie in the geodesic $[G(x), G(y)]$.

As a consequence of the above proposition, if the map $G$ mentioned in Remark 9.10 is injective and maps geodesics into geodesics, then it must be an isometry and the boundary map $f$ must be a snowflake map.

In the proof below we strongly use the density assumption (c). That (c) cannot be dropped from the assumptions of Proposition 9.11 is obvious, and it is easy to construct an example showing that (d) cannot be dropped either. The following example shows that neither (a) nor (b) can be dropped.

Example 9.12. Let $X$ be a binary rooted tree and $Y$ be a ternary rooted tree. We inductively map $X$ to $Y$ as follows: The root $0_{X}$ is mapped to $0_{Y}$, and with $x_{1}$ and $x_{2}$ being the children of $0_{X}$, we set $G\left(x_{1}\right)=y_{1}$ and $G\left(x_{2}\right)=0_{Y}$, where $y_{1}, y_{2}$ and $y_{3}$ are the three children of $0_{Y}$. With $x_{2,1}$ and $x_{2,2}$ being the children of $x_{2}$, we set $G\left(x_{2,1}\right)=y_{2}$ and $G\left(x_{2,2}\right)=y_{3}$. Repeating this procedure for the regular subtrees rooted at $x_{1}, x_{2,1}$, and $x_{2,2}$, we extend $G$ to the next generation. Iterating this process, we obtain a rough quasiisometry $G: X \rightarrow Y$ satisfying

$$
\frac{1}{2}|x-y|-2 \leq|G(x)-G(y)| \leq|x-y| \text { for } x, y \in X .
$$

(The worst case being when both $x$ and $y$ are mapped to the same vertices as their parents, and the same holds for every other of their ancestors.) Observe that $G$ is surjective and maps geodesics into geodesics. However, it is not injective, and there is no isometry between $\partial X$ and $\partial Y$, nor between $X$ and $Y$. Thus, the conclusion of Proposition 9.11 fails here, showing that the injectivity assumption cannot be dropped.

However, by Theorem 9.9, $G$ still induces a quasisymmetry between the boundaries $\partial X$ and $\partial Y$ with $\eta$ as in (9.9), $\alpha_{1}=\varepsilon_{Y} / 2 \varepsilon_{X}$ and $\alpha_{2}=\varepsilon_{Y} / \varepsilon_{X}$. Note that 
if we equip $\partial X$ and $\partial Y$ with the visual metrics given by (5.1) with $\varepsilon_{X}=\log 3$ and $\varepsilon_{Y}=\log 2$, then $\partial X$ can be identified with the usual ternary Cantor dust, while $\partial Y$ corresponds to a totally disconnected variant of the Sierpiński gasket, see Example 5.3, and that in this case $0<\alpha_{1}, \alpha_{2}<1$.

Furthermore, for $y \in Y$ let $H(y)$ be the smallest (with respect to $\leq$ ) $x \in X$ such that $G(x)=y$. Then $H: Y \rightarrow X$ is an order-preserving rough quasiisometry with $H\left(0_{Y}\right)=0_{X}$ which fulfills all the requirements in Proposition 9.11 but for (b) since the geodesic from $y_{2}$ to $y_{3}$ is mapped to $0_{Y}, x_{2,1}$ and $x_{2,2}$ which are not contained in any common geodesic. As $H$ is not an isometry, this shows that (b) cannot be dropped from Proposition 9.11.

Proof of Proposition 9.11. We use $\hat{x}$ to denote the parent of a vertex $x$. We seek to show that the children of $0_{X}$ map bijectively to the children of $0_{Y}$. Assume first that $G\left(0_{X}\right)=0_{Y}$ and let $x$ be a child of $0_{X}$. If $\widehat{G(x)} \neq 0_{Y}$, then by the density assumption (c) and the fact that each vertex of $Y$ has at least two children, there exists $t \in X$ such that $G(t)>\widehat{G(x)}$ and $G(t)$ is not in the geodesic ray containing $0_{Y}$ and $G(x)$. In other words, $G(x), G(t)$ and $0_{Y}$ cannot belong to any geodesic in $Y$. On the other hand, it is always possible to find a geodesic in $X$ containing $x, t$ and $0_{X}$. This violates (b), and hence $G(x)$ must be a child of $0_{Y}$. Conversely, if $y$ is a child of $0_{Y}$, then there exists $u \in X$ such that $G(u)>y$, by the density assumption (c) again. Let $a$ be the child of $0_{X}$ which is an ancestor of $u$, and $a^{\prime}$ be another child of $0_{X}$. By the above, $G(a)$ and $G\left(a^{\prime}\right)$ are children of $0_{Y}$. If $G(a) \neq y \neq G\left(a^{\prime}\right)$, then $G(u), G(a)$ and $G\left(a^{\prime}\right)$ do not belong to any geodesic in $Y$, but it is always possible to find a geodesic in $X$ containing $u, a$ and $a^{\prime}$, which is a contradiction. Thus $y=G(a)$ or $y=G\left(a^{\prime}\right)$. Hence the children of $0_{X}$ map bijectively to the children of $0_{Y}$.

Next, we proceed by induction. Assume that for all $z \in X$ with $1 \leq|z| \leq n$, we have that $G(z)$ is a child of $G(\hat{z})$, and let $x \in X$ be arbitrary with $|x|=n+1 \geq 2$. We distinguish two cases:

Case 1. If $G(x)>G(\hat{x})$ is not a child of $G(\hat{x})$ then as above, by the density assumption (c), we can find $v \in X$ such that $G(x)$ and $G(v)$ are not comparable with respect to $\leq$ and their largest common ancestor is the parent of $G(x)$. Then $G(x), G(\hat{x})$ and $G(v)$ do not belong to any geodesic in $Y$, but $x, \hat{x}$ and $v$ belong to a geodesic in $X$. This is a contradiction.

Case 2. If $G(x) \ngtr G(\hat{x})$ then, as the ancestors of $G(\hat{x})$ are exactly the images of the ancestors of $\hat{x}$ (by the induction hypothesis), we see that $G(x)$ and $G(\hat{x})$ are not comparable with respect to the partial ordering. Let $z$ be their largest common ancestor. If $z \neq 0_{Y}$ then the image of the geodesic from $0_{X}$ to $x$ must contain $G(x)$, $G(\hat{x})$ and $0_{Y}$, which is impossible. On the other hand, if $z=0_{Y}$, then by the above, $G(x)$ is not a child of $0_{Y}$, i.e $z$ is not the parent of $G(x)$. Again by the density assumption (c), we can find $w \in X$ such that $G(w)$ and $G(x)$ are not comparable with respect to the partial ordering, but their largest common ancestor is the parent of $G(x)$. Thus, the vertices $G(x), G(\hat{x})$ and $G(w)$ do not belong to any geodesic in $Y$ but there exists a geodesic in $X$ containing $x, \hat{x}$ and $w$. This final contradiction shows that $G(x)$ must be a child of $G(\hat{x})$. By induction, this holds for all $x \in X$ and hence $|G(x)-G(y)|=|x-y|$ for all $x, y \in X$.

To show that $G$ is surjective, let $y^{\prime} \in Y$ be arbitrary. By the density assumption (c), there exists $x^{\prime} \in X$ such that $G\left(x^{\prime}\right)>y^{\prime}$. Since $\left|G\left(x^{\prime}\right)\right|=\left|x^{\prime}\right|$, we can find $t^{\prime} \leq x^{\prime}$ such that $\left|y^{\prime}\right|=\left|t^{\prime}\right|$. By the above, we have $G\left(t^{\prime}\right) \leq G\left(x^{\prime}\right)$ and $\left|G\left(t^{\prime}\right)\right|=\left|t^{\prime}\right|=\left|y^{\prime}\right|$, showing that $G\left(t^{\prime}\right)=y^{\prime}$. Thus $G$ is surjective, and hence an isometry.

Finally, if $G\left(0_{X}\right) \neq 0_{Y}$ then let $Y^{\prime}$ be the tree $Y$ rerooted at $G\left(0_{X}\right)$. Then $Y$ and $Y^{\prime}$ are isometric with respect to $|\cdot-\cdot|$. Let $G^{\prime}: X \rightarrow Y^{\prime}$ be the map induced 
by $G$. Note that $G^{\prime}\left(0_{X}\right)=0_{Y^{\prime}}$ and that each vertex in $Y^{\prime}$ has at least two children. Thus we can apply the above result to $G^{\prime}$ to show that $G^{\prime}$ is an isometry, which is equivalent to $G$ being an isometry.

\section{References}

1. Beardon, A. and Minda, D., Sphere-preserving maps in inversive geometry, Proc. Amer. Math. Soc. 130 (2002), 987-998.

2. Bellissard, J. and Pearson, J., Noncommutative Riemannian geometry and diffusion on ultrametric Cantor sets, J. Noncommut. Geom. 3 (2009), 447-480.

3. Bennett, C. and Sharpley, R., Interpolation of Operators, Pure and Applied Mathematics 129, Academic Press, Boston, MA, 1988.

4. BJörn, A. and BJörn, J., Nonlinear Potential Theory on Metric Spaces, EMS Tracts in Mathematics 17, European Math. Soc., Zurich, 2011.

5. Buörn, A., Buörn, J. and Shanmugalingam, N., The Dirichlet problem for $p$-harmonic functions on metric spaces, J. Reine Angew. Math. 556 (2003), 173-203.

6. Buörn, A., Buörn, J. and Shanmugalingam, N., Sobolev extensions of Hölder continuous and characteristic functions on metric spaces, Canadian J. Math. 59 (2007), 1135-1153.

7. Björn, J. and Shanmugalingam, N., Poincaré inequalities, uniform domains, and extension properties for Newton-Sobolev spaces in metric spaces, J. Math. Anal. Appl. 332 (2007), 190-208.

8. Bonk, M., Heinonen, J. and Koskela, P., Uniformizing Gromov Hyperbolic Spaces, Astérisque 270 (2001).

9. Bourdon, M. and Pajot, H., Rigidity of quasi-isometries for some hyperbolic buildings, Comment. Math. Helv. 75 (2000), 701-736.

10. Bourdon, M. and PAJot, H., Quasi-conformal geometry and hyperbolic geometry, in Rigidity in Dynamics and Geometry (Cambridge, 2000), pp. 1-17, Springer, Berlin, 2002.

11. Bourdon, M. and Pajot, H., Cohomologie $l_{p}$ et espaces de Besov, J. Reine Angew. Math. 558 (2003), 85-108.

12. Bridson, M. and Haffliger, A., Metric Spaces of Non-positive Curvature, Grundlehren der Mathematischen Wissenschaften 319, Springer, Berlin, 1999.

13. Dalrymple, K., Strichartz, R. and Vinson, J., Fractal differential equations on the Sierpiński gasket, J. Fourier Anal. Appl. 5 (1999), 203-284.

14. Danielli, D., Garofalo, N. and Nhieu, D.-M., Sub-elliptic Besov spaces and the characterization of traces on lower dimensional manifolds, in Harmonic Analysis and Boundary Value Problems (Fayetteville, AR, 2000), Contemp. Math. 277, pp. 19-37, Amer. Math. Soc., Providence, RI, 2001.

15. Danielli, D., Garofalo, N. and Nhieu, D.-M., Non-doubling Ahlfors Measures, Perimeter Measures, and the Characterization of the Trace Spaces of Sobolev Functions in Carnot-Carathéodory spaces, Mem. Amer. Math. Soc. 182:857 (2006).

16. Falconer, K., Techniques in Fractal Geometry, Wiley, Chichester, 1997.

17. FArb, B. and Mosher, L., A rigidity theorem for the solvable BaumslagSolitar groups, Invent. Math. 131 (1998), 419-451.

18. Garnett, J., Positive length but zero analytic capacity, Proc. Amer. Math. Soc. 24 (1970), 696-699; errata, ibid. 26 (1970), 701.

19. Gogatishvili, A., Koskela, P. and Shanmugalingam, N., Interpolation properties of Besov spaces defined on metric spaces, Math. Nachr. 283 (2010), $215-231$. 
20. Gromov, M., Hyperbolic groups, in Essays in Group Theory, Math. Sci. Res. Inst. Publ. 8, pp. 75-263, Springer, New York, 1987.

21. HajŁasz, P., Sobolev spaces on metric-measure spaces, in Heat Kernels and Analysis on Manifolds, Graphs and Metric Spaces (Paris, 2002), Contemp. Math. 338, pp. 173-218, Amer. Math. Soc., Providence, RI, 2003.

22. Hajeasz, P. and Koskela, P., Sobolev met Poincaré, Mem. Amer. Math. Soc. 145:688 (2000).

23. Hajzasz, P. and Martio, O., Traces of Sobolev functions on fractal type sets and characterization of extension domains, J. Funct. Anal. 143 (1997), 221-246.

24. Hambly, B. and Kumagai, T., Heat kernel estimates for symmetric random walks on a class of fractal graphs and stability under rough isometries, in Fractal Geometry and Applications: a Jubilee of Benoit Mandelbrot, Part 2, Proc. Sympos. Pure Math. 72, pp. 233-259, Amer. Math. Soc., Providence, RI, 2004.

25. Heinonen, J., Lectures on Analysis on Metric Spaces, Universitext, Springer, New York, 2001.

26. Heinonen, J., Kilpeläinen, T. and Martio, O., Harmonic morphisms in nonlinear potential theory, Nagoya Math. J. 125 (1992), 115-140.

27. Heinonen, J., KilpeläInen, T. and Martio, O., Nonlinear Potential Theory of Degenerate Elliptic Equations, 2nd ed., Dover, Mineola, NY, 2006.

28. Heinonen, J. and Koskela, P., Quasiconformal mappings in metric spaces with controlled geometry, Acta Math. 181 (1998), 1-61.

29. Herman, P. E., Peirone, R. and Strichartz, R. S., p-energy and $p$ harmonic functions on Sierpiński gasket type fractals, Potential Anal. 20 (2004), $125-148$.

30. Herron, D., Shanmugalingam, N. and Xie, X., Uniformity from Gromov hyperbolicity, Illinois J. Math. 52 (2008), 1065-1109.

31. Ivanov, L. D., Variations of Sets and Functions, Nauka, Moscow, 1975 (Russian).

32. Jeffers, J., Lost theorems of geometry, Amer. Math. Monthly 107 (2000), $800-812$

33. Jonsson, A. and Wallin, H., The trace to subsets of $\mathbb{R}^{n}$ of Besov spaces in the general case, Anal. Math. 6 (1980), 223-254.

34. Jonsson, A. and Wallin, H., Function Spaces on Subsets of $\mathbf{R}^{n}$, Math. Rep. 2:1, Harwood, London, 1984.

35. Kapovich, M., Hyperbolic Manifolds and Discrete Groups, Progress in Mathematics 183, Birkhäuser, Boston, 2001.

36. Kigami, J., Dirichlet forms and associated heat kernels on the Cantor set induced by random walks on trees, Adv. Math. 225 (2010), 2674-2730.

37. Korte, R., Geometric implications of the Poincaré inequality, Results Math. 50 (2007), 93-107.

38. Koskela, P., YAng, D. and Zhou, Y., Pointwise characterizations of Besov and Triebel-Lizorkin spaces and quasiconformal mappings, Adv. Math. 226 (2011), 3579-3621.

39. MAz'YA, V. G., Sobolev Spaces with Applications to Elliptic Partial Differential Equations, Springer, Heidelberg, 2011.

40. Semmes, S., An introduction to the geometry of ultrametric spaces, Preprint, 2007, arXiv:0711.0709.

41. Semmes, S., Cellular structures, quasisymmetric mappings, and spaces of homogeneous type, Preprint, 2007, arXiv:0711.1333.

42. Strichartz, R. S., Some properties of Laplacians on fractals, J. Funct. Anal. 164 (1999), 181-208.

43. Strichartz, R. S. and Wong, C., The $p$-Laplacian on the Sierpiński gasket, Nonlinearity 17 (2004), 595-616. 
44. Shanmugalingam, N., Newtonian spaces: An extension of Sobolev spaces to metric measure spaces, Rev. Mat. Iberoam. 16 (2000), 243-279.

45. Shanmugalingam, N., Harmonic functions on metric spaces, Illinois J. Math. 45 (2001), 1021-1050.

46. Shanmugalingam, N. and Xie, X., A rigidity property of some negatively curved solvable Lie groups, Comment. Math. Helv. 87 (2012), 805-823.

47. Triebel, H., Theory of Function Spaces, Monographs in Mathematics 78, Birkhäuser, Basel, 1983. 